Resolubilidade global de uma classe de campos vetoriais

$$
\text { Rafael Borro Gonzalez }
$$



Data de Depósito:

Assinatura:

\title{
Resolubilidade global de uma classe de campos vetoriais
}

\author{
Rafael Borro Gonzalez
}

Orientador: Prof. Dr. Adalberto Panobianco Bergamasco

Dissertação apresentada ao Instituto de Ciências Matemáticas e de Computação - ICMC-USP, como parte dos requisitos para obtenção do título de Mestre em Ciências - Matemática. VERSÃO REVISADA.

USP - São Carlos

Fevereiro/2011 
Ficha catalográfica elaborada pela Biblioteca Prof. Achille Bassi e Seção Técnica de Informática, ICMC/USP, com os dados fornecidos pelo(a) autor(a)

Borro Gonzalez, Rafael
Resolubilidade global de uma classe de campos
vetoriais / Rafael Borro Gonzalez; orientador
Adalberto Panobianco Bergamasco -- São Carlos, 2011.
95 p.
Dissertação (Mestrado - Programa de Pós-Graduação en
Matemática) -- Instituto de Ciências Matemáticas e
de Computação, Universidade de São Paulo, 2011.
1. Resolubilidade global. 2. Campos vetoriais. 3.
Soluços periódicas. I. Panobianco Bergamasco,
Adalberto, orient. II. Título.


Aos meus pais,

Marcos e

Dora. 

À Deus, por me abençoar com um trabalho que me faz feliz.

Aos meus pais, Marcos e Dora, que sempre me apoiaram e me incentivaram a estudar.

Agradeço à minha avó Janir, pela acolhida, carinho e pelos cuidados durante os anos de graduação.

Também agradeço à minha avó Levina, por todas as orações.

Sou muitíssimo grato ao meu orientador e amigo Adalberto, pelos valiosos ensinamentos sobre matemátca que tem me fornecido durante esses anos que estamos trabalhando juntos.

Também sou grato aos meus amigos Carlos, Andreza, Nelson, Vinícius, Apoenã, Thaís e Alex; foi muito bom construir conhecimento ao lado deles. Agradeço à minha namorada Ana, pelo amor que tem me dado e pelos momentos de descontração que me proporciona.

Por fim, agradeço o apoio financeiro da FAPESP. 

O tema em estudo é a resolubilidade global de campos vetoriais em $\mathbb{T}_{(x, t)}^{2}$ da forma $\mathrm{L}=\partial_{t}+a(x) \partial_{x}$, onde $a \in \mathcal{C}^{\infty}\left(\mathbb{T}^{1}\right)$ é uma função real. Consideraremos o caso em que o operador $L$ age no espaço de funções e o caso em que $L$ age no espaço de distribuições. Utilizando teoria de distribuições, forneceremos condições necessárias e suficientes para que a imagem de $\mathrm{L}$ seja um subespaço fechado, ou seja, para que $L$ seja globalmente resolúvel. O caso mais interessante ocorre quando a função $a$ se anula em algum ponto mas não é identicamente nula; neste caso, L será globalmente resolúvel se, e somente se, $a^{-1}(0)$ contiver apenas zeros de ordem finita. Faremos também o estudo da resolubilidade global de operadores da forma $\mathrm{P}=\partial_{t}+\partial_{x}(a \cdot)$, os quais são perturbações por um termo de ordem zero dos campos da forma $L$. Os operadores da forma $P$ surgem quando consideramos o transposto de um operador da forma $L$. 

The topic under study is the global solvability of vector fields of the form $\mathrm{L}=\partial_{t}+a(x) \partial_{x}$ on the 2 -torus $\mathbb{T}_{(x, t)}^{2}$, where $a \in \mathcal{C}^{\infty}\left(\mathbb{T}^{1}\right)$ is a real valued function. We consider the operator $L$ acting on both spaces of functions and distributions. Using distribution theory we give necessary and sufficient conditions for the closedness of the range of $\mathrm{L}$, ie, for $\mathrm{L}$ to be globally solvable. The most interesting case occurs when $a$ vanishes somewhere but not everywhere; in this case, we show that a necessary and sufficient condition for $L$ to be globally solvable is that each zero of $a$ is of finite order. We also study the global solvability of operators of the form $\mathrm{P}=\partial_{t}+\partial_{x}(a \cdot)$, which are perturbations of $L$ by a term of zero order. The operators $P$ appear when we consider the transpose operator of $L$. 

1 Preliminares $\quad 13$

I Espaços vetoriais topológicos . . . . . . . . . . . . . . . . . . 13

2 Teoria das distribuições $\quad 19$

I Funções-teste . . . . . . . . . . . . . . . . . . . . . . . . . . . . . . 19

II Distribuições . . . . . . . . . . . . . . . . . . . . . . . 27

III Distribuições no toro . . . . . . . . . . . . . . . . . . . 46

IV Série de Fourier e produto tensorial . . . . . . . . . . . . 54

3 Resolubilidade global $\quad 59$

I Resolubilidade global no espaço de funções . . . . . . . . . . . . . . . 61

II Resolubilidade global no espaço de distribuições . . . . . . . . . . . . . . 79

A Campos vetoriais com coeficientes constantes no toro bidimensional 87

$\begin{array}{ll}\text { Referências Bibliográficas } & 91\end{array}$

$\begin{array}{ll}\text { Índice Remissivo } & 93\end{array}$

$\begin{array}{ll}\text { Índice de Notações } & 95\end{array}$ 

O estudo da resolubilidade local de EDP lineares de tipo principal (por exemplo, campos vetoriais reais ou complexos) recebeu um grande impulso em 1963 com a publicação do artigo [13] de Nirenberg e Treves; nesse trabalho foi formulada pela primeira vez a condição $(\mathrm{P})$, a qual (conforme foi demonstrado após um bom número de artigos e de anos) é necessária e suficiente para a resolubilidade local de operadores do tipo $\mathrm{L}=\partial_{t}+a(x) \partial_{x}$, onde $a \in \mathcal{C}^{\infty}\left(\mathbb{T}^{1}\right)$ é uma função real. Na maioria de tais resultados, trata-se da existência de soluções fracas (distribuições) quando o segundo membro é de classe $\mathcal{C}^{\infty}$.

Para o estudo da resolubilidade global, interpretaremos um campo vetorial em $\mathbb{T}^{2}$ como um operador $L: \mathcal{C}^{\infty}\left(\mathbb{T}^{2}\right) \rightarrow \mathcal{C}^{\infty}\left(\mathbb{T}^{2}\right)$. Assim, o transposto de $L$ será um operador agindo no espaço de distribuições $\mathcal{D}^{\prime}\left(\mathbb{T}^{2}\right)$. Também faz sentido considerar o operador $L$ agindo no espaço das distribuições $\mathcal{D}^{\prime}\left(\mathbb{T}^{2}\right)$. Em ambos os casos, diremos que $L$ será globalmente resolúvel quando sua imagem for um subespaço fechado. Utilizando resultados de Análise Funcional, verifica-se que a imagem de $L$ será um subespaço fechado se, e somente se, a imagem de $L$ for um subespaço que contenha o ortogonal ao núcleo do transposto ${ }^{t} L$. Dessa forma, $L$ será globalmente resolúvel se, e somente se, para cada $f$ que estiver no ortogonal ao núcleo do transposto, existir $u$ tal que $L u=f$.

Neste trabalho, estudaremos a resolubilidade global de uma determinada classe de campos vetoriais reais em $\mathbb{T}_{(x, t)}^{2}$, a saber, $\mathrm{L}=\partial_{t}+a(x) \partial_{x}$, onde $a \in \mathcal{C}^{\infty}\left(\mathbb{T}^{1}\right)$ é uma função real. Se $a$ for a função identicamente nula ou se $a$ for uma função que não se anula em $\mathbb{T}^{1}$, então o estudo da resolubilidade global de $\mathrm{L}$ se reduz ao estudo da resolubilidade global de campos vetoriais com coeficientes constantes. Quando a se anular em algum ponto e não for identicamente nula, L será globalmente resolúvel se, e somente se, os zeros de $a$ forem todos de ordem finita. 
Quando calculamos o transposto de um operador da forma $L: \mathcal{C} \infty\left(\mathbb{T}^{2}\right) \rightarrow \mathcal{C}^{\infty}\left(\mathbb{T}^{2}\right)$, obtemos ${ }^{t} \mathrm{~L}: \mathcal{D}^{\prime}\left(\mathbb{T}^{2}\right) \rightarrow \mathcal{D}^{\prime}\left(\mathbb{T}^{2}\right)$, onde ${ }^{t} \mathrm{~L}=\partial_{t}+\partial_{x}(a \cdot)$. Também será interessante analisar a resolubilidade global dos operadores da forma $\mathrm{P}=\partial_{t}+\partial_{x}(a \cdot)$, os quais são perturbações por um termo de ordem zero dos operadores da forma L. Para a classe de operadores da forma $P$, obtemos os mesmos resultados que aparecem na resolubilidade global dos operadores da forma L.

Diremos que um operador $L: \mathcal{C}^{\infty}\left(\mathbb{T}^{2}\right) \rightarrow \mathcal{C}^{\infty}\left(\mathbb{T}^{2}\right)$ será fortemente resolúvel quando sua imagem for um subespaço de codimensão finita. Alguns resultados de Análise Funcional fornecem a seguinte caracterização para a resolubilidade forte (veja comentário após a Definição (1.2) de [4]): um operador $L: \mathcal{C}^{\infty}\left(\mathbb{T}^{2}\right) \rightarrow \mathcal{C}^{\infty}\left(\mathbb{T}^{2}\right)$ será fortemente resolúvel se, e somente se, a imagem de $L$ for um subespaço fechado e o núcleo do operador transposto ${ }^{t} L$ for um subespaço de dimensão finita.

Além da resolubilidade global, analisaremos se os operadores das formas L e P são fortemente resolúveis; analisaremos também se tais operadores são sobrejetores. 


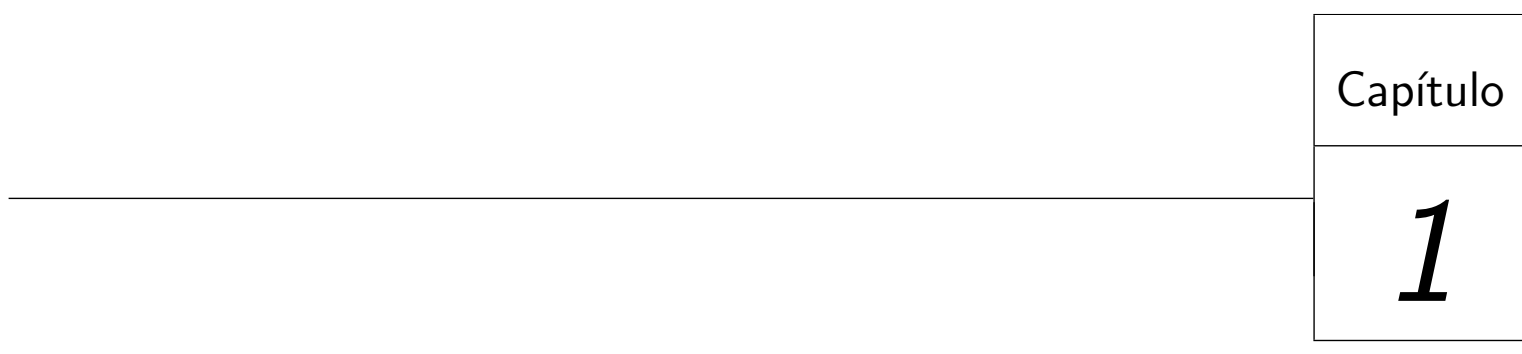

Preliminares

No presente capítulo, apresentaremos alguns resultados básicos de Análise Funcional, os quais serão utilizados no estudo da resolubilidade global de certos campos vetoriais que realizaremos posteriormente.

\section{Espaços vetoriais topológicos}

Adotaremos a Definição (1.6) da referência [15] para espaços vetoriais topológicos; a saber, um espaço vetorial topológico será um espaço vetorial $X$ munido de uma topologia $\tau$ que satisfaz as seguintes condições:

(i) Para cada $x \in X$, o conjunto $\{x\}$ é fechado.

(ii) A adição

$$
X \times X \ni(x, y) \mapsto x+y \in X
$$

e a multiplicação por escalar

$$
\mathbb{C} \times X \ni(\alpha, x) \mapsto \alpha x \in X
$$

são contínuas, considerando em $X$ a topologia $\tau$ e considerando a topologia produto em $X \times X$ e $\mathbb{C} \times X$. 
Desta forma, todo espaço vetorial topológico será um espaço de Hausdorff, conforme Teorema (1.12) de [15].

Denotaremos por $X^{\prime}$ o espaço dual topológico de um espaço vetorial topológico $X$. Em $X^{\prime}$ existem algumas topologias canônicas, as quais tornam $X^{\prime}$ um espaço vetorial topológico localmente convexo (veja a definição de espaço vetorial topológico localmente convexo em [15]).

As duas topologias mais importantes no nosso estudo são as chamadas topologia fraca* e a topologia forte. A definição da topologia fraca* pode ser consultada no item 3.14 da referência [15] e a definição da topologia forte pode ser consultada na página 198 da referência [17]. Observamos que na referência [17] a topologia que chamamos de fraca* é chamada apenas de topologia fraca, a qual está definida na página 197.

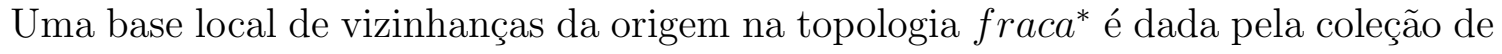
conjuntos da forma $V_{\epsilon}\left(x_{1}, \ldots, x_{n}\right)=\left\{x^{\prime} \in X^{\prime} ;\left|\left\langle x^{\prime}, x_{j}\right\rangle\right|<\epsilon, j=1, \ldots, n\right\}$, qualquer que seja $\left\{x_{1}, \ldots, x_{n}\right\}$ um subconjunto finito de $X$ e qualquer que seja $\epsilon>0$ um número real. Para a topologia forte, uma base local de vizinhanças da origem é dada pela coleção de conjuntos da forma $W(B)=\left\{x^{\prime} \in X^{\prime} ; \sup _{x \in B}\left|\left\langle x^{\prime}, x\right\rangle\right| \leq 1\right\}$, qualquer que seja $B$ um subconjunto limitado de $X$. O conjunto $W(B)$ é chamado o polar de $B$.

O espaço dual munido da topologia $f r a c a^{*}$ será denotado por $X_{\sigma}^{\prime}$ e o espaço dual munido da topologia forte será denotado por $X_{b}^{\prime}$.

Pode-se comparar a topologia rraca* $^{*}$ com a topologia forte, obtendo que a topologia forte é mais fina que a topologia fraca*.

Dados $M \subset X$ e $N \subset X^{\prime}$ subespaços vetoriais, definimos os anuladores de $M$ e $N$ por ${ }^{\circ} M=\left\{x^{\prime} \in X^{\prime} ;\left\langle x^{\prime}, x\right\rangle=0\right.$, se $\left.x \in M\right\}$ e $N^{\circ}=\left\{x \in X ;\left\langle x^{\prime}, x\right\rangle=0\right.$, se $\left.x^{\prime} \in N\right\}$, respectivamente.

É fácil ver que $N^{\circ} \subset X$ é um subespaço vetorial fechado, pois $N^{\circ}=\bigcap_{x^{\prime} \in N} x^{\prime-1}(0)$. Também pode-se provar que ${ }^{\circ} M \subset X^{\prime}$ é um subespaço vetorial fechado na topologia fraca*; de fato, se considerarmos a topologia fraca* em $X^{\prime}$, então cada $x^{\prime \prime} \in\left(X_{\sigma}^{\prime}\right)^{\prime}$ é da forma $J(x) \in X^{\prime \prime}$, onde $J(x)$ é o funcional linear contínuo dado por $\left\langle J(x), x^{\prime}\right\rangle=\left\langle x^{\prime}, x\right\rangle$, para todo $x^{\prime} \in X^{\prime}$ (veja 3.14 e o Teorema 3.10 da referência [15]). A identidade ${ }^{\circ} M=$ $\bigcap_{x \in M} \operatorname{ker} J(x)$ implica que ${ }^{\circ} M \subset X^{\prime}$ é um subespaço fechado na topologia fraca* $^{*}$.

Quando $X$ for um espaço vetorial localmente convexo, a aplicação $J: X \rightarrow\left(X_{\sigma}^{\prime}\right)^{\prime}$ será um isomorfismo linear entre os espaços vetoriais $X$ e $\left(X_{\sigma}^{\prime}\right)^{\prime}$. Tal afirmação é dada pela Proposição (35.1) da referência [17]. 
Segue da Proposição (19.6) da referência [17] que podemos considerar $J: X \rightarrow\left(X_{b}^{\prime}\right)^{\prime}$ e pela Proposição (19.7) da referência [17] segue que $J: X \rightarrow\left(X_{b}^{\prime}\right)^{\prime}$ será injetora, sempre que $X$ for um espaço vetorial topológico localmente convexo.

Um espaço vetorial topológico localmente convexo $X$ será dito semireflexivo quando $J: X \rightarrow\left(X_{b}^{\prime}\right)^{\prime}$ for sobrejetora, isto é, quando $J$ for um isomorfismo entre os espaços vetoriais $X$ e $\left(X_{b}^{\prime}\right)^{\prime}$. Diremos que um espaço vetorial topológico localmente convexo $X$ será reflexivo se $J$ for um homeomorfismo linear (isomorfismo na categoria de espaço vetorial topológico) entre $X$ e $\left(X_{b}^{\prime}\right)_{b}^{\prime}$.

O fecho de um subconjunto $Z \subset X^{\prime}$ na topologia fraca* $^{*}$ será denotado por $\bar{Z}^{\omega^{*}}$ e o fecho de $Z$ na topologia forte será denotado por $\bar{Z}$.

Diretamente da definição de anulador obtemos $M \subset\left({ }^{\circ} M\right)^{\circ}$ e como $\left({ }^{\circ} M\right)^{\circ}$ é fechado, temos $\bar{M} \subset\left({ }^{\circ} M\right)^{\circ}$. Por outro lado, se $X$ for localmente convexo, uma aplicação do Teorema de Hanh-Banach ( veja (3.5) de [15] ) garante que vale a igualdade $\bar{M}=\left({ }^{\circ} M\right)^{\circ}$, pois se existir $x_{0} \in\left({ }^{\circ} M\right)^{\circ} \backslash \bar{M}$, então existirá $x^{\prime} \in X^{\prime} \operatorname{com}\left\langle x^{\prime}, x_{0}\right\rangle \neq 0$ e $x^{\prime} \in{ }^{\circ} M$, o que é uma contradição.

Também é imediato verificar que $N \subset{ }^{\circ}\left(N^{\circ}\right)$ e como ${ }^{\circ}\left(N^{\circ}\right) \subset X^{\prime}$ é fechado na topologia fraca $^{*}$, daí temos $\bar{N}^{\omega^{*}} \subset{ }^{\circ}\left(N^{\circ}\right)$. Como $X^{\prime}$ munido da topologia fraca* é sempre localmente convexo, se existir $x_{0}^{\prime} \in{ }^{\circ}\left(N^{\circ}\right) \backslash \bar{N}^{\omega^{*}}$, segue novamente do Teorema (3.5) de [15] que existirá $J(x) \in X^{\prime \prime}$ tal que $\left\langle x_{0}^{\prime}, x\right\rangle=\left\langle J(x), x_{0}^{\prime}\right\rangle \neq 0$ e $\left\langle x^{\prime}, x\right\rangle=\left\langle J(x), x^{\prime}\right\rangle=0$, para todo $x^{\prime} \in N$. Então $x \in N^{\circ}$ e $\left\langle x_{0}^{\prime}, x\right\rangle \neq 0$; uma contradição com a hipótese de que $x_{0}^{\prime} \in{ }^{\circ}\left(N^{\circ}\right)$.

As considerações acima demonstram a seguinte proposição.

1.1 Proposição. Seja $X$ um espaço vetorial topológico. Para quaisquer $M \subset X e$ $N \subset X^{\prime}$ subespaços vetoriais, temos

(i) $\bar{M} \subset\left({ }^{\circ} M\right)^{\circ}$ e vale a igualdade $\bar{M}=\left({ }^{\circ} M\right)^{\circ}$ sempre que $X$ for localmente convexo.

(ii) $\bar{N}^{\omega^{*}}={ }^{\circ}\left(N^{\circ}\right)$.

Sejam $X$ e $Y$ dois espaços vetoriais topológicos. A cada transformação linear contínua $T: X \rightarrow Y$ podemos associar, de maneira natural, uma transformação linear entre os espaços duais $Y^{\prime}$ e $X^{\prime}$. Tal transformação linear é dita o transposto de $T$ e definida por ${ }^{t} T\left(y^{\prime}\right) \doteq y^{\prime} \circ T$, para todo $y^{\prime} \in Y^{\prime}$. Vale deixar registrado que ${ }^{t} T$ é uma transformação linear contínua, considerando em ambos os duais $Y^{\prime}$ e $X^{\prime}$ a topologia fraca* ou considerando em ambos os duais a topologia forte, conforme o corolário da Proposição (19.5) da referência [17]. 
A próxima proposição fornece duas relações entre os núcleos e as imagens de $T$ e ${ }^{t} T$.

1.2 Proposição. Seja $T: X \rightarrow Y$ uma transformação linear contínua entre os espaços vetoriais topológicos localmente convexos $X$ e $Y$. Então

(i) $\left(\operatorname{ker}^{t} T\right)^{\circ}=\overline{\mathcal{R}(T)}$, onde $\mathcal{R}(T)$ denota a imagem da transformação $T$.

(ii) ${ }^{\circ}(\operatorname{ker} T)={\overline{\mathcal{R}\left({ }^{t} T\right)}}^{\omega^{*}}$.

Prova: (i) Das definições de anulador e da definição de transposto segue que $\operatorname{ker}^{t} T=$ ${ }^{\circ} \mathcal{R}(T)$. Pela Proposição (1.1) temos $\operatorname{ker}\left({ }^{t} T\right)^{\circ}=\overline{\mathcal{R}(T)}$.

(ii) O fato de $Y$ ser localmente convexo implica que $Y^{\prime}$ separa pontos em $Y$ (veja o corolário do Teorema (3.4) da referência [15]). Utilizando tal resultado é possível verificar que $\operatorname{ker} T=\mathcal{R}\left({ }^{t} T\right)^{\circ}$ e da Proposição $(1.1)$ segue que ${ }^{\circ}(\operatorname{ker} T)={\overline{\mathcal{R}}\left({ }^{t} T\right)}^{\omega^{*}}$.

Quando $X$ for um espaço vetorial topológico, cuja topologia seja induzida por uma métrica invariante e completa, diremos que $X$ será um $F$-espaço. Se $X$ for um $F$-espaço localmente convexo, então $X$ será dito um espaço de Fréchet.

1.3 Proposição. Sejam $X$ um F-espaço, $Y$ um espaço de Fréchet e $T: X \rightarrow Y$ uma transformação linear contínua com imagem fechada em $Y$. Então ${ }^{t} T: Y^{\prime} \rightarrow X^{\prime}$ possui imagem fraca* fechada em $X^{\prime}$.

Prova: Segue da Proposição (1.2) que basta demonstrarmos a inclusão

$$
{ }^{\circ}(\operatorname{ker} T) \subset \mathcal{R}\left({ }^{t} T\right)
$$

Tomemos então $x^{\prime} \in{ }^{\circ}(\operatorname{ker} T)$. Nosso objetivo é exibir $y^{\prime} \in Y^{\prime}$ tal que ${ }^{t} T y^{\prime}=x^{\prime}$; o que ocorre se, e somente se, $\left\langle y^{\prime}, T x\right\rangle=\left\langle{ }^{t} T y^{\prime}, x\right\rangle=\left\langle x^{\prime}, x\right\rangle$, para todo $x \in X$.

Considere $\Delta: \mathcal{R}(T) \rightarrow \mathbb{C}$ o funcional linear dado por $\Delta T x=\left\langle x^{\prime}, x\right\rangle$, para todo $x \in X$. Note que $\Delta$ está bem definido, pois $x^{\prime} \in{ }^{\circ}(\operatorname{ker} T)$.

Se $\Delta$ for um funcional linear contínuo, o fato de $Y$ ser localmente convexo implicará que existe $y^{\prime} \in Y^{\prime}$ tal que $y^{\prime}=\Delta$ em $\mathcal{R}(T)$, conforme o Teorema (3.6) da referência [15], o qual é uma consequência do Teorema de Hahn-Banach. Assim, $\left\langle{ }^{t} T y^{\prime}, x\right\rangle=\left\langle y^{\prime}, T x\right\rangle=$ $\Delta T x=\left\langle x^{\prime}, x\right\rangle$, para todo $x \in X$, ou seja, ${ }^{t} T y^{\prime}=x^{\prime}$ e então $x^{\prime} \in \mathcal{R}\left({ }^{t} T\right)$.

Verifiquemos agora que $\Delta$ é contínuo, ou seja, que $\Delta$ é contínuo na origem. Observe que $\mathcal{R}(T)$ é um espaço de Fréchet, pois por hipótese $\mathcal{R}(T)$ é um subespaço fechado do espaço de Fréchet $Y$. Assim, do Corolário (2.12) da referência [15], o qual é uma consequência do Teorema da Aplicação Aberta, segue que $T: X \rightarrow \mathcal{R}(T)$ é uma aplicação 
aberta. Como $x^{\prime}: X \rightarrow \mathbb{C}$ é contínuo, dado $\epsilon>0$, existe um aberto $U \subset X$ que contém a origem e satisfaz $x^{\prime}(U) \subset B(0, \epsilon) \subset \mathbb{C}, \log 0 T(U) \subset \mathcal{R}(T)$ é um aberto que satisfaz $0=T(0) \in T(U)$ e $\Delta(T(U))=x^{\prime}(U) \subset B(0, \epsilon)$, o que prova a continuidade de $\Delta$.

Algumas vezes será interessante conhecer o bitransposto de uma transformação linear $T: X \rightarrow Y$, onde $X$ e $Y$ são espaços vetoriais topológicos. Podemos considerar o bitransposto de $T$ como o transposto de ${ }^{t} T: Y_{\sigma}^{\prime} \rightarrow X_{\sigma}^{\prime}$ ou como o transposto de ${ }^{t} T: Y_{b}^{\prime} \rightarrow X_{b}^{\prime}$. Denotaremos o bitransposto de $T$ por ${ }^{t t} T$.

Na próxima proposição, destacaremos uma propriedade envolvendo os anuladores dos núcleos do operador $T$ e do seu bitransposto ${ }^{t t} T$.

1.4 Proposição. Sejam $T: X \rightarrow Y$ uma transformação linear contínua entre espaços vetoriais localmente convexos e ${ }^{t} T: Y_{\sigma}^{\prime} \rightarrow X_{\sigma}^{\prime}$ seu transposto. Então ${ }^{t t} T:\left(X_{\sigma}^{\prime}\right)^{\prime} \rightarrow\left(Y_{\sigma}^{\prime}\right)^{\prime}$ satisfaz $^{\circ}(\operatorname{ker} T)=\left(\operatorname{ker}^{t t} T\right)^{\circ}$.

Prova: Utilizando o fato de que ambas as aplicações canônicas $J_{X}: X \rightarrow\left(X_{\sigma}^{\prime}\right)^{\prime}$ e $J_{Y}: Y \rightarrow\left(Y_{\sigma}^{\prime}\right)^{\prime}$ são isomorfismos entre espaços vetoriais, obtemos as seguintes propriedades:

(i) $T=J_{Y}^{-1} \circ{ }^{t t} T \circ J_{X}$.

(ii) $\operatorname{ker}\left(J_{Y}^{-1} \circ{ }^{t t} T \circ J_{X}\right)=J_{X}^{-1}\left(\operatorname{ker}^{t t} T\right)$.

(iii) ${ }^{\circ}\left[\operatorname{ker}\left(J_{Y}^{-1} \circ{ }^{t t} T \circ J_{X}\right)\right]=\left(\operatorname{ker}^{t t} T\right)^{\circ}$.

Por fim, (i) e (iii) implicam que ${ }^{\circ}(\operatorname{ker} T)=\left(\operatorname{ker}^{t t} T\right)^{\circ}$.

Vale observar que a única propriedade utilizada na demonstração da proposição acima é o fato de que $J_{X}$ e $J_{Y}$ são isomorfismos na categoria de espaços vetoriais. Utilizando a mesma demonstração obtemos a proposição a seguir.

1.5 Proposição. Seja $T: X \rightarrow Y$ uma transformação linear contínua entre espaços vetoriais localmente convexos e semireflexivos. Considere o transposto ${ }^{t} T: Y_{b}^{\prime} \rightarrow X_{b}^{\prime}$. Então ${ }^{t t} T:\left(X_{b}^{\prime}\right)^{\prime} \rightarrow\left(Y_{b}^{\prime}\right)^{\prime}$ satisfaz $^{\circ}(\operatorname{ker} T)=\left(\operatorname{ker}^{t t} T\right)^{\circ}$. 



$\frac{-1}{2}$

\section{Teoria das distribuições}

A teoria das distribuições é fundamental no estudo da resolubilidade global que desenvolveremos no capítulo seguinte. Neste segundo capítulo, apresentaremos as ferramentas da teoria das distribuições necessárias para tal estudo.

\section{Funções-teste}

Começaremos introduzindo algumas notações que são utilizadas na teoria de distribuições. Um subconjunto aberto arbitrário de $\mathbb{R}^{N}$ será denotado por $\Omega$ e um intervalo aberto arbitrário de $\mathbb{R}$ será denotado por $I$. Denotaremos por $\mathcal{C}^{\infty}(\Omega)$ o espaço vetorial formado pelas funções a valores complexos, definidas e infinitamente diferenciáveis em $\Omega$, isto é, $\phi \in \mathcal{C}^{\infty}(\Omega)$ se $\partial^{\alpha} \phi=\frac{\partial^{\alpha_{1}}}{\partial x_{1}^{\alpha_{1}}}\left(\cdots\left(\frac{\partial^{\alpha_{N}}}{\partial x_{N}^{\alpha_{N}}} \phi\right)\right)=\partial_{x_{1}}^{\alpha_{1}}\left(\cdots\left(\partial_{x_{N}}^{\alpha_{N}} \phi\right)\right)$ existir e for contínua, para cada multi-índice $\alpha$ de inteiros nã̃o negativos. O suporte de cada função $f \in \mathcal{C}^{\infty}(\Omega)$ será denotado por $S(f)$.

O subespaço de $\mathcal{C}^{\infty}(\Omega)$ formado pelas funções que possuem suporte compacto será denotado por $\mathcal{C}_{c}^{\infty}(\Omega)$ e $\mathcal{P}_{T}\left(\mathbb{R}^{N}\right)$ denotará o subespaço de $\mathcal{C}^{\infty}\left(\mathbb{R}^{N}\right)$ formado pelas funções $T$-periódicas em cada variável, onde $0<T \in \mathbb{R}$.

Se $x=\left(x_{1}, \ldots, x_{N}\right) \in \Omega \subset \mathbb{R}^{N}, \phi \in \mathcal{C}^{\infty}(\Omega)$ e $\alpha=\left(\alpha_{1}, \ldots, \alpha_{N}\right), \beta=\left(\beta_{1}, \ldots, \beta_{N}\right)$ são dois multi-índices em $\mathbb{Z}^{N}$, escrevemos 


$$
\begin{gathered}
\alpha \leq \beta, \text { se } \alpha_{j} \leq \beta_{j}, \text { para cada } j=1, \ldots, N, \\
|\alpha|=\sum_{j=1}^{N}\left|\alpha_{j}\right| \\
x^{\alpha}=\prod_{j=1}^{N} x_{j}^{\alpha_{j}} .
\end{gathered}
$$

Se $\alpha, \beta \in \mathbb{Z}_{+}^{N}$, escrevemos

$$
\begin{gathered}
\alpha !=\prod_{j=1}^{N} \alpha_{j} ! \\
\left(\begin{array}{l}
\beta \\
\alpha
\end{array}\right)=\frac{\beta !}{\alpha !(\beta-\alpha) !}=\prod_{j=1}^{N}\left(\begin{array}{c}
\beta_{j} \\
\alpha_{j}
\end{array}\right), \text { se } \alpha \leq \beta,
\end{gathered}
$$

Vale também destacar duas ferramentas da análise muito utilizadas na teoria das distribuições. A regra de Leibniz,

$$
\partial^{\alpha}(\phi \psi)=\sum_{\beta \leq \alpha}\left(\begin{array}{l}
\alpha \\
\beta
\end{array}\right)\left(\partial^{\beta} \phi\right)\left(\partial^{\alpha-\beta} \psi\right)
$$

onde $\phi, \psi \in \mathcal{C}^{\infty}(\Omega)$ e a fórmula de Taylor com resto integral, dada por

$$
\phi(x+y)=\sum_{|\alpha|<k}\left(\partial^{\alpha} \phi\right)(x) y^{\alpha} / \alpha !+k \int_{0}^{1}(1-t)^{k-1} \sum_{|\alpha|=k}\left(\partial^{\alpha} \phi\right)(x+t y) y^{\alpha} / \alpha ! d t
$$

onde $\phi$ é uma função $\mathbb{C}^{k}$ em uma vizinhança do segmento $[x, x+y] \subset \mathbb{R}^{N}$.

Em cada um dos espaços vetoriais descritos anteriormente existe uma topologia que os tornam espaços vetoriais localmente convexos e completos. Em $\mathcal{P}_{T}\left(\mathbb{R}^{N}\right)$ fixaremos a topologia induzida pela família de seminormas

$$
\rho_{k}(\theta)=\sup _{\substack{x \in \mathbb{R}^{N} \\|\alpha| \leq k}}\left|\left(\partial^{\alpha} \theta\right)(x)\right|
$$

onde $k \in \mathbb{N}$. Segue do Teorema (1.37) da referência [15] que tal topologia é localmente convexa, cada seminorma $\rho_{k}$ é contínua e uma base local de vizinhanças da origem é dada pelas interseções finitas de conjuntos da forma $V\left(\rho_{k}, n\right)=\left\{\theta \in \mathcal{P}_{T}\left(\mathbb{R}^{N}\right) ; \rho_{k}(\theta)<1 / n\right\}$, com $k, n \in \mathbb{N}$. Do Teorema (1.24) da referência [15] segue que tal topologia é metrizável e da Observação (1.38) (c) de [15] segue que tal topologia coincide com a topologia 
induzida pela métrica

$$
d(\theta, \tilde{\theta})=\sum_{k=1}^{\infty} \frac{\rho_{k}(\theta-\tilde{\theta})}{2^{k}\left(1+\rho_{k}(\theta-\tilde{\theta})\right)} .
$$

Verifica-se também que $\mathcal{P}_{T}\left(\mathbb{R}^{N}\right)$ é completo, ou seja, um espaço de Fréchet.

A próxima proposição caracteriza a convergência das sequências em $\mathcal{P}_{T}\left(\mathbb{R}^{N}\right)$. Sua demonstração pode ser consultada na página 3 da referência [18].

2.1 Proposição. Uma sequência $\left(\theta_{n}\right)_{n \in \mathbb{N}}$ converge para a função nula em $\mathcal{P}_{T}\left(\mathbb{R}^{N}\right)$ se, e somente se, $\left(\rho_{k}\left(\theta_{n}\right)\right)_{n \in \mathbb{N}}$ converge para zero em $\mathbb{C}$, para cada $k \in \mathbb{N}$, isto é, $\left(\partial^{\alpha} \theta_{n}\right)$ converge uniformemente em $\mathbb{R}^{N}$ para a função nula, para cada $\alpha \in \mathbb{Z}_{+}^{N}$.

O espaço vetorial $\mathcal{C}^{\infty}(\Omega)$ também possui uma topologia localmente convexa, metrizável e completa. Seja $\left(K_{j}\right)_{j \in \mathbb{N}}$ uma sequência de conjuntos compactos que forma uma exaustão do aberto $\Omega \subset \mathbb{R}^{N}$, isto é, para cada $j \in \mathbb{N}$ temos $K_{j} \subset K_{j+1}$ e $\Omega=\cup_{j \in \mathbb{N}} K_{j}$ (a Proposição (4) da página 278 da referência [5] garante que tal exaustão existe). Para cada $j \in \mathbb{N}$, defina a seminorma

$$
\sigma_{j}(\phi)=\sup _{x \in K_{j},|\alpha| \leq j}\left|\left(\partial^{\alpha} \phi\right)(x)\right|
$$

Como anteriormente, tais seminormas induzem uma topologia localmente convexa e metrizável, com relação a qual cada seminorma $\sigma_{j}$ é contínua. Uma base local de vizinhanças da origem é dada pela família de conjuntos da forma $V_{j}=\left\{\phi \in \mathcal{C}^{\infty}(\Omega) ; \sigma_{j}(\phi)<1 / j\right\}$ e $\mathcal{C}^{\infty}(\Omega)$ munido de tal topologia é completo, conforme o Exemplo (1.46) da referência $[15]$.

Existe uma maneira de caracterizar a convergência das sequências em $\mathcal{C}^{\infty}(\Omega)$. Se uma sequência $\left(\phi_{n}\right)_{n \in \mathbb{N}}$ converge para a função nula em $\mathcal{C}^{\infty}(\Omega)$, a continuidade de cada seminorma $\sigma_{j}$ fornece que $\left(\sigma_{j}\left(\phi_{n}\right)\right)_{n \in \mathbb{N}}$ converge para zero em $\mathbb{C}$, para cada $j \in \mathbb{N}$. Reciprocamente, suponha que $\left(\sigma_{j}\left(\phi_{n}\right)\right)_{n \in \mathbb{N}}$ converge para zero em $\mathbb{C}$, para todo $j \in \mathbb{N}$. Então, para cada vizinhança da origem $V_{j_{0}}$, existe $n_{0} \in \mathbb{N}$ tal que $\sigma_{j_{0}}\left(\phi_{n}\right)<1 / j_{0}$, para todo $n \geq n_{0}$; isto é, $\phi_{n} \in V_{j_{0}}$, para todo $n \geq n_{0}$ e, assim, $\left(\phi_{n}\right)_{n \in \mathbb{N}}$ converge para a função nula em $\mathcal{C}^{\infty}(\Omega)$. Por fim, o fato de $\left(\sigma_{j}\left(\phi_{n}\right)\right)_{n \in \mathbb{N}}$ convergir para zero em $\mathbb{C}$, para todo $j \in \mathbb{N}$, é equivalente a dizer que cada sequência $\left(\partial^{\alpha} \phi_{n}\right)_{n \in \mathbb{N}}$ converge uniformemente em $K$ para a função nula, quaisquer que sejam $\alpha \in \mathbb{Z}_{+}^{N}$ e $K \subset \Omega$ um compacto.

Os argumentos acima demonstram a seguinte proposição. 
2.2 Proposição. Uma sequência $\left(\phi_{n}\right)_{n \in \mathbb{N}}$ converge para a função nula em $\mathcal{C}^{\infty}(\Omega)$ se, e somente se, para cada $\alpha \in \mathbb{Z}_{+}^{N}$, a sequência $\left(\partial^{\alpha} \phi_{n}\right)_{n \in \mathbb{N}}$ converge uniformemente para a função nula em subconjuntos compactos de $\Omega$.

Descreveremos agora uma topologia em $\mathcal{C}_{c}^{\infty}(\Omega)$. Considere $\left(K_{j}\right)_{j \in \mathbb{N}}$ uma exaustão de $\Omega \subset \mathbb{R}^{N}$ por subconjuntos compactos e seja $\mathcal{C}_{c}^{\infty}\left(K_{j}\right)$ o subespaço de $\mathcal{C}_{c}^{\infty}(\Omega)$ formado pelas funções que possuem suporte contido em $K_{j}$. Uma sequência de seminormas em $\mathcal{C}_{c}^{\infty}(\Omega)$ é dada por

$$
\|\phi\|_{n}=\sup _{\substack{x \in \Omega ; \\|\alpha| \leq n}}\left|\left(\partial^{\alpha} \phi\right)(x)\right|, \quad n \in \mathbb{Z}_{+} .
$$

Restringindo a sequência de seminormas $\left(\|\cdot\|_{n}\right)_{n \in \mathbb{Z}_{+}}$a cada espaço $\mathcal{C}_{c}^{\infty}\left(K_{j}\right)$ e considerando em $\mathcal{C}_{c}^{\infty}\left(K_{j}\right)$ a topologia induzida por tal sequência de seminormas, obtemos, segundo os resultados (1.46) e (6.2) da referência [15], uma sequência crescente de espaços de Fréchet $\mathcal{C}_{c}^{\infty}\left(K_{1}\right) \subset \cdots \subset \mathcal{C}_{c}^{\infty}\left(K_{j}\right) \subset \mathcal{C}_{c}^{\infty}\left(K_{j+1}\right) \subset \cdots$ onde a topologia de $\mathcal{C}_{c}^{\infty}\left(K_{j}\right)$ coincide com a topologia induzida de $\mathcal{C}_{c}^{\infty}\left(K_{j+1}\right)$, uma vez que uma base local de vizinhanças da origem em $\mathcal{C}_{c}^{\infty}\left(K_{j}\right)$ é dada pela coleção $V_{n}=\left\{\phi \in \mathcal{C}_{c}^{\infty}\left(K_{j}\right) ;\|\phi\|_{n}<1 / n\right\}$, $n \in \mathbb{N}$.

Como $\mathcal{C}_{c}^{\infty}(\Omega)=\bigcup_{j \in \mathbb{N}} \mathcal{C}_{c}^{\infty}\left(K_{j}\right)$, existe uma única topologia localmente convexa em $\mathcal{C}_{c}^{\infty}(\Omega)$, chamada de topologia limite indutivo (veja Apêndice B.2 da referência [9]), com relação a qual uma base local convexa e equilibrada da origem é dada pela família $\mathcal{B}$ de conjuntos convexos e equilibrados $W \subset \mathcal{C}_{c}^{\infty}(\Omega)$, tais que $W \cap \mathcal{C}_{c}^{\infty}\left(K_{j}\right)$ é um subconjunto aberto de $\mathcal{C}_{c}^{\infty}\left(K_{j}\right)$, para todo $j \in \mathbb{N}$ (veja a definição de subconjunto equilibrado na referência [15]). A demonstração de tal afirmação pode ser encontrada em (6.3) e (6.4) da referência [15], uma vez que a família $\mathcal{B}$ coincide com a família formada pelos conjuntos convexos e equilibrados $W^{\prime} \subset \mathcal{C}_{c}^{\infty}(\Omega)$ tais que $W^{\prime} \cap \mathcal{C}_{c}^{\infty}(K)$ é aberto em $\mathcal{C}_{c}^{\infty}(K)$, para todo compacto $K \subset \Omega$. Segue da demonstração do Teorema (6.5)(a) de [15] que cada inclusão $i_{K}: \mathcal{C}_{c}^{\infty}(K) \rightarrow \mathcal{C}_{c}^{\infty}(\Omega)$ é contínua. Mais ainda, tal topologia é a topologia localmente convexa mais fina em $\mathcal{C}_{c}^{\infty}(\Omega)$ que torna cada inclusão $i_{K}$ contínua.

A convergência das sequências em $\mathcal{C}_{c}^{\infty}(\Omega)$ se caracteriza conforme o teorema abaixo, o qual encontra-se demonstrado no Teorema (6.5) da referência [15].

2.3 Teorema. Uma sequência $\left(\phi_{n}\right)_{n \in \mathbb{N}}$ converge para a função nula em $\mathcal{C}_{c}^{\infty}(\Omega)$ se, e somente se, existe um compacto $K \in \Omega$ que contém o suporte de cada $\phi_{n}$ e cada sequência $\left(\partial^{\alpha} \phi_{n}\right)_{n \in \mathbb{N}}$ converge uniformemente em $\Omega$ para a função nula, onde $\alpha \in \mathbb{Z}_{+}^{N}$. 
2.4 Observação. A topologia limite indutivo em $\mathcal{C}_{c}^{\infty}(\Omega)$ não é metrizável, mas isso não é relevante no que diz respeito à continuidade de um funcional linear definido em $\mathcal{C}_{c}^{\infty}(\Omega)$. Tal afirmação é uma consequência do Teorema (6.6) da referência [15], que afirma o seguinte: se $Y$ for um espaço localmente convexo e se $T: \mathcal{C}_{c}^{\infty}(\Omega) \rightarrow Y$ for uma transformação linear, então $T$ será contínua se, e somente se, $\left(T\left(\phi_{n}\right)\right)_{n \in \mathbb{N}}$ convergir para zero em $Y$, para cada sequencia $\left(\phi_{n}\right)_{n \in \mathbb{N}}$ que converge para a função nula em $\mathcal{C}_{c}^{\infty}(\Omega)$.

A partir de uma função $\phi \in \mathcal{C}^{\infty}(\Omega)$ e um compacto $K \subset \Omega$, podemos obter uma função em $\mathcal{C}_{c}^{\infty}(\Omega)$ que coincida com $\phi$ em uma vizinhança do compacto $K$. Tal resultado é uma consequência do seguinte lema, conhecido como lema da função de corte, cuja demonstração pode ser consultada na página 25 da referência [11].

2.5 Lema. Seja $K \subset \Omega \subset \mathbb{R}^{N}$ um compacto. Então existe $\phi \in \mathcal{C}_{c}^{\infty}(\Omega)$, tal que $0 \leq \phi \leq 1$ e $\phi \equiv 1$ em uma vizinhança de $K$.

O próximo teorema fornece uma maneira de escrever uma função $\phi \in \mathcal{C}_{c}^{\infty}(\Omega)$ como uma soma de funções com suportes menores. Sua demonstração pode ser consultada na página 27 da referência [11].

2.6 Teorema. (i) Sejam $\left\{\Omega_{j}\right\}_{j \in J}$ uma familia de subconjuntos abertos de $\mathbb{R}^{N}, \Omega=$ $\bigcup_{j \in J} \Omega_{j}$ e $\phi \in \mathcal{C}_{c}^{\infty}(\Omega)$. Para cada $j \in J$, existe $\phi_{j} \in \mathcal{C}_{c}^{\infty}\left(\Omega_{j}\right)$ com $\phi_{j} \not \equiv 0$ apenas para um número finito de índices $j \in J$, satisfazendo

$$
\phi=\sum_{j \in J} \phi_{j}
$$

Se $\phi$ for não negativa, podemos tomar cada $\phi_{j}$ também não negativa.

(ii) Sejam $\left\{\Omega_{j}\right\}_{j \in J}$ uma familia de subconjuntos abertos de $\mathbb{R}^{N}$ e $K$ um subconjunto compacto de $\bigcup_{j \in J} \Omega_{j}$. Para cada $j \in J$, existe $0 \leq \phi_{j} \in \mathcal{C}_{c}^{\infty}\left(\Omega_{j}\right)$, com apenas um número finito não identicamente nula, satisfazendo

$$
\sum_{j \in J} \phi_{j} \leq 1
$$

com igualdade em uma vizinhança de $K$.

O próximo teorema é um resultado atribuido a Borel. 
2.7 Teorema. Sejam $\left(v_{j}\right)_{j \in \mathbb{Z}_{+}} \subset \mathcal{P}_{2 \pi}(\mathbb{R}), x_{0} \in \mathbb{R}$ e $A \subset \mathbb{R}$ um aberto contendo $x_{0}$. Então existe $r>0$ e uma função $v(x, t)$ em $\mathcal{C}^{\infty}(A \times \mathbb{R})$ que é $2 \pi$-periódica na variável $t$, satisfaz $S(v) \subset\left[x_{0}-r, x_{0}+r\right] \times \mathbb{R} \subset A \times \mathbb{R} e$

$$
\left(\partial_{x}^{j} v\right)\left(x_{0}, t\right)=v_{j}(t)
$$

para todo $t \in \mathbb{R}$ e todo $j \in \mathbb{Z}_{+}$.

Prova: Utilizando a translação $x \mapsto x-x_{0}$ se necessário, podemos supor que $x_{0}=0$.

Sejam $r \in(0,1)$ tal que $[-r, r] \subset A$ e $g \in \mathcal{C}_{c}^{\infty}((-r, r))$ constante igual a 1 em uma vizinhança de $\left[0, \frac{r}{2}\right]$.

Para cada $j \in \mathbb{Z}_{+}$e $(x, t) \in A \times \mathbb{R}$, defina

$$
g_{j}(x, t)=\frac{g\left(\frac{x}{\epsilon_{j}}\right) x^{j} v_{j}(t)}{j !}
$$

onde, a priori, $\epsilon_{j} \in(0,1)$.

Cada função $g_{j}$ pertence a $\mathcal{C}^{\infty}(A \times \mathbb{R})$, pois existem e são contínuas todas as derivadas parciais. Mais ainda, $S\left(g_{j}\right) \subset\left(-r \epsilon_{j}, r \epsilon_{j}\right) \times \mathbb{R}$ e $g_{j}(x, t)=g_{j}(x, t+2 \pi n)$, para todo $n \in \mathbb{Z}$, já que $v_{j} \in \mathcal{P}_{2 \pi}(\mathbb{R})$.

O próximo passo é estimar $\left|\left(\partial^{\alpha} g_{j}\right)(x, t)\right|$, para $j>1$ e $\alpha=\left(\alpha_{x}, \alpha_{t}\right) \in \mathbb{Z}_{+}^{2}$ com $|\alpha|<j-1$. Para $(x, t) \in\left(-r \epsilon_{j}, r \epsilon_{j}\right) \times \mathbb{R}$, temos

$$
\begin{gathered}
\left|\left(\partial^{\alpha} g_{j}\right)(x, t)\right|=\frac{1}{j !}\left|\partial^{\alpha_{t}} v_{j}(t) \partial^{\alpha_{x}}\left(g\left(x / \epsilon_{j}\right) x^{j}\right)\right|= \\
\frac{1}{j !}\left|\partial^{\alpha_{t}} v_{j}(t)\right| \cdot\left|\sum_{l \leq \alpha_{x}}\left(\begin{array}{c}
\alpha_{x} \\
l
\end{array}\right) \partial^{l}\left(x^{j}\right) \partial^{\alpha_{x}-l}\left(g\left(x / \epsilon_{j}\right)\right)\right|= \\
\frac{1}{j !}\left|\partial^{\alpha_{t}} v_{j}(t)\right| \cdot\left|\sum_{l \leq \alpha_{x}}\left(\begin{array}{c}
\alpha_{x} \\
l
\end{array}\right) \frac{j !}{(j-l) !} x^{j-l} \cdot \epsilon_{j}^{l-\alpha_{x}}\left(\partial^{\alpha_{x}-l} g\right)\left(x / \epsilon_{j}\right)\right| \leq \\
\left\|\partial^{\alpha_{t}} v_{j}\right\|_{\infty} \alpha_{x} ! \sum_{l \leq \alpha_{x}} r^{j-l} \epsilon_{j}^{j-\alpha_{x}}\left\|\partial^{\alpha_{x}-l} g\right\|_{\infty} \leq \\
\left\|\partial^{\alpha_{t}} v_{j}\right\|_{\infty} \alpha_{x} ! \epsilon_{j}^{j-\alpha_{x}} \sum_{l \leq \alpha_{x}}\left\|\partial^{\alpha_{x}-l} g\right\|_{\infty} \leq \\
C_{\alpha, j} \epsilon_{j}^{j-\alpha_{x}},
\end{gathered}
$$

em que $C_{\alpha, j}$ é uma constante que independe de $(x, t) \in\left(-r \epsilon_{j}, r \epsilon_{j}\right) \times \mathbb{R}$ e $\epsilon_{j}$. 
Como $S\left(g_{j}\right) \subset\left(-r \epsilon_{j}, r \epsilon_{j}\right) \times \mathbb{R}$, temos

$$
\left|\left(\partial^{\alpha} g_{j}\right)(x, t)\right| \leq C_{\alpha, j} \epsilon_{j}^{j-\alpha_{x}}
$$

para $j>1, \alpha=\left(\alpha_{x}, \alpha_{t}\right) \in \mathbb{Z}_{+}^{2}$ com $|\alpha|<j-1$ e qualquer $(x, t) \in A \times \mathbb{R}$.

Escolhendo cada $\epsilon_{j} \in(0,1)$ suficientemente pequeno, obtemos

$$
\left|\left(\partial^{\alpha} g_{j}\right)(x, t)\right| \leq 2^{-j}
$$

para $j>1, \alpha=\left(\alpha_{x}, \alpha_{t}\right) \in \mathbb{Z}_{+}^{2}$ com $|\alpha|<j-1$ e qualquer $(x, t) \in A \times \mathbb{R}$.

Segue da estimativa acima que, para cada $\alpha \in \mathbb{Z}_{+}^{2}$, a série $\sum_{j=0}^{\infty}\left(\partial^{\alpha} g_{j}\right)$ é uniformemente convergente em $A \times \mathbb{R}$. Definindo

$$
v(x, t)=\sum_{j=0}^{\infty} g_{j}(x, t)
$$

para $(x, t) \in A \times \mathbb{R}$, e utilizando um resultado básico de análise (veja o Teorema (1.1.5) da referência [11]) segue que $v$ é uma função de $\mathcal{C}^{\infty}(A \times \mathbb{R})$; mais ainda, $S(v) \subset(-r, r) \times \mathbb{R}$ e

$$
\left(\partial_{x}^{j} v\right)(0, t)=\sum_{k=0}^{\infty}\left(\partial_{x}^{j} g_{k}\right)(0, t)
$$

para todo $t \in \mathbb{R}$ e $j \in \mathbb{Z}_{+}$.

Utilizando a regra de Leibniz verifica-se que

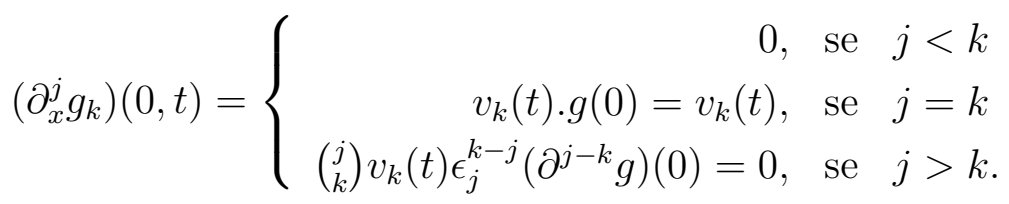

Assim, a função $v(x, t) \in \mathcal{C}^{\infty}(A \times \mathbb{R})$ é $2 \pi-$ periódica na variável $t$, está suportada em $(-r, r) \times \mathbb{R}$ e satisfaz $\left(\partial_{x}^{j} v\right)(0, t)=v_{j}(t)$, para todo $t \in \mathbb{R}$ e $j \in \mathbb{Z}_{+}$.

A ordem de anulamento é um fator importante quando desejamos dividir uma função $f \in \mathcal{C}^{\infty}(I)$ por uma função $g \in \mathcal{C}^{\infty}(I)$. As próximas duas proposições consideram o caso em que $g$ possui apenas zeros de ordem finita e fornecem condições suficientes para que possamos dividir $f$ por $g$. 
2.8 Proposição. (i) Se $g \in \mathcal{C}^{\infty}(I)$ for uma função que se anula apenas em $x_{0} \in I$, o qual é um zero de ordem finita $n \geq 1$ e $f \in \mathcal{C}^{\infty}(I)$ for uma função que se anula em $x_{0}$ de ordem maior que $n$, então existirá $h \in \mathcal{C}^{\infty}(I)$ satisfazendo $g h=f$, $f^{-1}(0)=h^{-1}(0)$ e a ordem de anulamento de $h$ em cada zero $x \in h^{-1}(0)$ será igual à ordem de anulamento de $f$ menos a ordem de anulamento de $g$ em $x$.

(ii) Se $g \in \mathcal{P}_{T}(\mathbb{R})$ possuir apenas zeros de ordem finita, $f \in \mathcal{P}_{T}(\mathbb{R})$ for uma função tal que $g^{-1}(0) \subset f^{-1}(0)$ e se a ordem de anulamento de $f$ em cada $x \in g^{-1}(0)$ for maior que a ordem de anulamento da função $g$, então existirá $h \in \mathcal{P}_{T}(\mathbb{R})$ tal que $g h=f, f^{-1}(0)=h^{-1}(0)$ e a ordem de anulamento de $h$ em cada zero $x \in h^{-1}(0)$ será igual à ordem de anulamento de $f$ menos a ordem de anulamento de $g$ em $x$.

Prova: (i) Utilizando a fórmula de Taylor com resto integral podemos escrever $g(x)=$ $\left(x-x_{0}\right)^{n} h_{n}(x)$ e $f(x)=\left(x-x_{0}\right)^{m} h_{m}(x)$, para todo $x \in I$, onde $h_{n}, h_{m} \in \mathcal{C}^{\infty}(I), h_{n}$ não se anula em $I, m>n$ e $m$ é menor ou igual à ordem de anulamento de $f$ em $x_{0}$. Assim, $h(x) \doteq\left(x-x_{0}\right)^{m-n} h_{m}(x) / h_{n}(x) \in \mathcal{C}^{\infty}(I)$ e satisfaz $g h=f$. Observe que $\left(x-x_{0}\right)^{m_{1}-n} h_{m_{1}}(x)=\left(x-x_{0}\right)^{m_{2}-n} h_{m_{2}}(x) \in \mathcal{C}^{\infty}(I)$, quaisquer que sejam $m_{1}, m_{2}>n$ e menores ou iguais à ordem de anulamento de $f$ em $x_{0}$. Diretamente da expressão que define $h$ segue que $h^{-1}(0)=f^{-1}(0)$ e que a ordem de anulamento de $h$ em $x_{0}$ é igual à ordem de anulamento de $f$ menos a ordem de anulamento de $g$ em $x_{0}$. Por fim, se $y \in h^{-1}(0) \backslash\left\{x_{0}\right\}$, então existe uma vizinhança de $y$ restrita a qual $g$ não se anula e da identidade $h=f / g$ segue que a ordem de anulamento de $h$ em $y$ é igual à ordem de anulamento de $f$ em $y$.

(ii) Fixe $x_{0} \in \mathbb{R} \backslash g^{-1}(0)$ e denote o conjunto $g^{-1}(0) \cap\left[x_{0}, x_{0}+T\right]$ por $\left\{x_{1}<\cdots<x_{r}\right\}$. Defina $x_{r+1} \doteq x_{0}+T$ e para $j=1, \ldots, r$ defina $I_{j}=\left(x_{j-1}, x_{j+1}\right)$. Segue do item $(i)$ que existe $h_{j} \in \mathcal{C}^{\infty}\left(I_{j}\right)$ tal que $g h_{j}=f$ em $I_{j}, h_{j}^{-1}(0)=f^{-1}(0) \cap I_{j}$ e a ordem de anulamento de $h_{j}$ em cada $x \in h_{j}^{-1}(0)$ é igual à ordem de anulamento de $f$ menos a ordem de anulamento de $g$ em $x$. Definindo $\tilde{h}(x)=h_{j}(x)$, se $x \in I_{j}$ e $\tilde{h}\left(x_{0}\right)=$ $\tilde{h}\left(x_{0}+T\right)=f\left(x_{0}\right) / g\left(x_{0}\right)$, segue que $\tilde{h} \in \mathcal{C}^{\infty}\left(\left(x_{0}, x_{0}+T\right)\right), g \tilde{h}=f$ em $\left[x_{0}, x_{0}+T\right]$, $\tilde{h}^{-1}(0)=f^{-1}(0) \cap\left[x_{0}, x_{0}+T\right]$ e a ordem de anulamento de $\tilde{h}$ em cada $x \in \tilde{h}^{-1}(0)$ é igual à ordem de anulamento de $f$ menos a ordem de anulamento de $g$ em $x$. Por fim, a extensão $T$ - periódica da função $\tilde{h}$, isto é, a função definida por $h(x+k T)=\tilde{h}(x)$, para todo $x \in\left[x_{0}, x_{0}+T\right]$ e todo $k \in \mathbb{Z}$, satisfaz as condições requeridas.

Utilizando a fórmula de Taylor com resto integral também verifica-se a seguinte proposição. 
2.9 Proposição. Seja $f \in \mathcal{P}_{T}\left(\mathbb{R}^{2}\right)$ e seja $g \in \mathcal{P}_{T}(\mathbb{R})$ uma função que possua apenas zeros de ordem finita. Se $f$ for flat em $g^{-1}(0) \times \mathbb{R}$, então existirá $h \in \mathcal{P}_{T}\left(\mathbb{R}^{2}\right)$, flat em $g^{-1}(0) \times \mathbb{R}$ e tal que $g(x) h(x, t)=f(x, t)$, para todo $(x, t) \in \mathbb{R}^{2}$.

Cada função $\phi \in \mathcal{C}_{c}^{\infty}\left(\mathbb{R}^{N}\right)$ define uma função em $\mathcal{P}_{T}\left(\mathbb{R}^{N}\right)$, dada por $\theta(x)=$ $\sum_{m \in \mathbb{Z}^{N}} \phi(x-T m)$, a qual se escreve como uma soma finita em cada bola fechada centrada na origem. Mais ainda, se $K \subset \mathbb{R}^{N}$ for um compacto, então, para cada $r>0$, existirão multi-índices $m_{1}^{r}, \ldots, m_{n}^{r} \in \mathbb{Z}^{N}$ tais que $\sum_{m \in \mathbb{Z}^{N}} \phi(x-T m)=\phi\left(x-T m_{1}^{r}\right)+\cdots+\phi\left(x-T m_{n}^{r}\right)$ em $B[0, r]$, para toda $\phi \in \mathcal{C}_{c}^{\infty}\left(\mathbb{R}^{N}\right)$ com $S(\phi) \subset K$. Veja os argumentos nas páginas 3 e 4 da referência [18]. Em particular, a função $\sum_{m \in \mathbb{Z}^{N}} \phi(x-T m) \in \mathcal{P}_{T}\left(\mathbb{R}^{N}\right)$ satisfaz $\left(\partial^{\alpha}\left(\sum_{m \in \mathbb{Z}^{N}} \phi(x-T m)\right)=\sum_{m \in \mathbb{Z}^{N}}\left(\partial^{\alpha} \phi\right)(x-T m)\right.$, para todo $\alpha \in \mathbb{Z}_{+}^{N}$.

Considere $\mathcal{U}_{T}\left(\mathbb{R}^{N}\right)=\left\{\phi \in \mathcal{C}_{c}^{\infty}\left(\mathbb{R}^{N}\right) ; 1 \equiv \sum_{m \in \mathbb{Z}^{N}} \phi(x-T m)\right\} \subset \mathcal{C}_{c}^{\infty}\left(\mathbb{R}^{N}\right)$. O próximo exemplo ilustra que $\mathcal{U}_{T}(\mathbb{R})$ é um subespaço não vazio. Utilizando uma função $\xi \in \mathcal{U}_{T}(\mathbb{R})$, obtemos uma função $\phi \in \mathcal{U}_{T}\left(\mathbb{R}^{N}\right)$, dada por $\phi\left(x_{1}, \ldots, x_{N}\right)=\xi\left(x_{1}\right) \cdots \xi\left(x_{N}\right)$.

2.10 Exemplo. Dado $x_{0} \in \mathbb{R}$, existe $\xi \in \mathcal{U}_{T}(\mathbb{R})$ tal que $S(\xi) \subset\left(x_{0}-T, x_{0}+T\right)$ e $\xi(x)=1$ em uma vizinhança de $x_{0}$.

Prova: Utilizando composição com translações basta demonstrarmos o caso em que $x_{0}=0$.

Fixe $s$ e $r$ tais que $T / 2<s<r<T$. Tome também uma função $\phi \in \mathcal{C}_{c}^{\infty}(\mathbb{R})$ tal que $S(\phi) \subset(-r, r), \phi \geq 0$ e $\phi \equiv 1$ em uma vizinhança de $[-s, s]$. Assim, para cada $x \in \mathbb{R}$, a soma $\sum_{m \in \mathbb{Z}} \phi(x-T m)$ é finita em uma vizinhança de $x$, positiva, $T$ - periódica e $\phi(x)=\sum_{m \in \mathbb{Z}} \phi(x-T m)$ em uma vizinhança da origem.

Então, definindo $\xi(x) \doteq \phi(x) / \sum_{m \in \mathbb{Z}} \phi(x-T m)$ segue que $\xi \in \mathcal{U}_{T}(\mathbb{R}), S(\xi) \subset(-T, T)$ e $\xi(x) \equiv 1$ em uma vizinhança da origem.

\section{Distribuições}

De uma maneira geral, uma distribuição em $\Omega \subset \mathbb{R}^{N}$ é um funcional linear contínuo, definido em um espaço de funções-teste. Sendo assim, os duais $\mathcal{C}^{\infty}(\Omega)^{\prime}, \mathcal{P}_{T}^{\prime}\left(\mathbb{R}^{N}\right) \doteq$ $\mathcal{P}_{T}\left(\mathbb{R}^{N}\right)^{\prime}$ e $\mathcal{D}^{\prime}(\Omega) \doteq \mathcal{C}_{c}^{\infty}(\Omega)^{\prime}$ são espaços de distribuições. 
Podemos caracterizar as distribuições em $\mathcal{P}_{T}^{\prime}\left(\mathbb{R}^{N}\right)$ com o seguinte teorema, que encontra-se na página (9) da referência [18].

2.11 Teorema. Seja $u: \mathcal{P}_{T}\left(\mathbb{R}^{N}\right) \rightarrow \mathbb{C}$ um funcional linear. São equivalentes:

(i) ué contínuo, isto é, $u \in \mathcal{P}_{T}^{\prime}\left(\mathbb{R}^{N}\right)$.

(ii) Existem uma constante $C>0$ e $m \in \mathbb{Z}_{+}$tais que

$$
|\langle u, \theta\rangle| \leq C \sum_{|\alpha| \leq m} \sup _{x \in \mathbb{R}^{N}}\left|\left(\partial^{\alpha} \theta\right)(x)\right|
$$

para toda $\theta \in \mathcal{P}_{T}\left(\mathbb{R}^{N}\right)$.

O menor inteiro não negativo $m \in \mathbb{Z}_{+}$que satisfaz a condição $(i i)$ do teorema acima é dito a ordem da distribuição $u \in \mathcal{P}_{T}^{\prime}\left(\mathbb{R}^{N}\right)$.

Com o teorema anterior fica bastante fácil demonstrar que $\delta: \mathcal{P}_{T}\left(\mathbb{R}^{N}\right) \rightarrow \mathbb{C}$, definida por $\langle\delta, \theta\rangle \doteq \theta(0)$, é uma distribuição de $\mathcal{P}_{T}^{\prime}\left(\mathbb{R}^{N}\right)$, chamada de distribuição delta de Dirac.

As distribuições de $\mathcal{C}^{\infty}(\Omega)^{\prime}$ se caracterizam pelo Teorema (III.2.2) da referência [12], o qual está enunciado a seguir.

2.12 Teorema. Seja $u: \mathcal{C}^{\infty}(\Omega) \rightarrow \mathbb{C}$ um funcional linear. São equivalentes:

(i) $u$ é contínuo, isto é, $u \in \mathcal{C}^{\infty}(\Omega)^{\prime}$.

(ii) Existem um compacto $K \in \Omega$, uma constante $C>0$ e $m \in \mathbb{Z}_{+}$tais que

$$
|\langle u, \phi\rangle| \leq C \sum_{|\alpha| \leq m} \sup _{x \in K}\left|\left(\partial^{\alpha} \phi\right)(x)\right|
$$

para toda $\phi \in \mathcal{C}^{\infty}(\Omega)$.

O próximo teorema, $\left(\right.$ III.2.3) da referência [12], caracteriza as distribuições de $\mathcal{D}^{\prime}(\Omega)$.

2.13 Teorema. Seja $u: \mathcal{C}_{c}^{\infty}(\Omega) \rightarrow \mathbb{C}$ um funcional linear. São equivalentes:

(i) $u$ é contínuo, isto é, $u \in \mathcal{D}^{\prime}(\Omega)$.

(ii) Para cada compacto $K \in \Omega$, existem uma constante $C>0$ e $m \in \mathbb{Z}_{+}$tais que

$$
|\langle u, \phi\rangle| \leq C \sum_{|\alpha| \leq m} \sup _{x \in \Omega}\left|\left(\partial^{\alpha} \phi\right)(x)\right|
$$

para toda $\phi \in \mathcal{C}_{c}^{\infty}(K)$. Por definição $\mathcal{C}_{c}^{\infty}(K)$ é o espaço das funções $\phi \in \mathcal{C}_{c}^{\infty}(\Omega)$ que possuem suporte contido em $K$. 
Dizemos que uma distribuição $u \in \mathcal{C}_{c}^{\infty}(\Omega)$ possui ordem finita se existir $m \in \mathbb{Z}_{+}$ que satisfaça o item $(i i)$ do teorema anterior para todo compacto $K \in \Omega$. Neste caso, o menor entre tais inteiros é dito a ordem da distribuição $u \in \mathcal{C}_{c}^{\infty}(\Omega)$.

Embora o espaço $\mathcal{C}_{c}^{\infty}(\Omega)$ não seja metrizável, da Observação (2.4) segue que $u \in \mathcal{D}^{\prime}(\Omega)$ se, e somente se, $\left(\left\langle u, \phi_{n}\right\rangle\right)_{n \in \mathbb{N}}$ converge para zero em $\mathbb{C}$, para toda sequência $\left(\phi_{n}\right)_{n \in \mathbb{N}}$ que converge para a função nula em $\mathcal{C}_{c}^{\infty}(\Omega)$.

Também podemos definir a distribuição delta de Dirac em $\mathcal{C}_{c}^{\infty}(\Omega)$, onde $\Omega \subset \mathbb{R}^{N}$ é um aberto contendo a origem. De fato, segue do teorema anterior que $\delta: \mathcal{C}_{c}^{\infty}(\Omega) \rightarrow \mathbb{C}$, dada por $\langle\delta, \phi\rangle \doteq \phi(0)$, é uma distribuição de $\mathcal{D}^{\prime}(\Omega)$.

Como $\mathcal{D}^{\prime}(\Omega)$ e $\mathcal{P}_{T}^{\prime}\left(\mathbb{R}^{N}\right)$ são espaços duais, podemos torná-los espaços vetoriais topológicos locamente convexos, considerando a topologia fraca* $^{*}$ ou a topologia forte. A próxima proposição trata de uma propriedade das sequências em $\mathcal{D}^{\prime}(\Omega)$.

2.14 Proposição. Uma sequência $\left(u_{n}\right)_{n \in \mathbb{N}} \subset \mathcal{D}^{\prime}(\Omega)$ converge na topologia fraca* se, e somente se, $\left(u_{n}\right)_{n \in \mathbb{N}}$ converge na topologia forte.

Prova: Como a topologia forte é mais fina que a topologia $\mathrm{fraca}^{*}$, basta provar que toda sequência que converge na topologia fraca* também converge na topologia forte. Suponha que $\left(u_{n}\right)_{n \in \mathbb{N}}$ seja uma seqência que converge para a origem em $\mathcal{D}^{\prime}(\Omega)_{\sigma}$. Devemos mostrar que cada vizinhança básica da origem em $\mathcal{D}^{\prime}(\Omega)_{b}$ contém $u_{n}$ a partir de algum $n_{0}$. Uma vizinhança básica da origem em $\mathcal{D}^{\prime}(\Omega)_{b}$ é da forma

$$
\left\{u \in \mathcal{D}^{\prime}(\Omega) ; \sup _{\phi \in B}|\langle u, \phi\rangle| \leq 1\right\}
$$

onde $B \subset \mathcal{C}_{c}^{\infty}(\Omega)$ é um subconjunto limitado. Do Teorema (6.5) (item c) da referência [15] segue que existe um compacto $K \subset \Omega$, com interior não vazio e tal que $B \subset \mathcal{C}_{c}^{\infty}(K)$, o qual é um espaço de Fréchet cuja topologia coincide com a topologia de subespaço induzida de $\mathcal{C}_{c}^{\infty}(\Omega)$ (veja Teorema (6.5) (item b) de [15]). Mais ainda, dos exemplos (6.2) e (1.46) de [15] segue que todo subconjunto fechado e limitado em $\mathcal{C}_{c}^{\infty}(K)$ é compacto. Como $B \subset \mathcal{C}_{c}^{\infty}(K)$ é um subconjunto limitado, então $\bar{B} \subset \mathcal{C}_{c}^{\infty}(K)$ é um compacto. Do Teorema (V.8) da referência [14] segue que a sequência formada pela restrição das distribuições $u_{n}$ ao subespaço $\mathcal{C}_{c}^{\infty}(K)$ converge uniformemente em $\bar{B}$; ou seja, para qualquer $\epsilon>0$ existe $n_{0} \in \mathbb{N}$ tal que

$$
\sup _{\phi \in \bar{B}}\left|\left\langle u_{n}, \phi\right\rangle\right|<\epsilon,
$$


para todo $n>n_{0}$. Em particular, existe $n_{0} \in \mathbb{N}$ tal que

$$
u_{n} \in\left\{u \in \mathcal{D}^{\prime}(\Omega) ; \sup _{\phi \in B}|\langle u, \phi\rangle| \leq 1\right\}
$$

para todo $n>n_{0}$.

Existe um subespaço de $\mathcal{D}^{\prime}\left(\mathbb{R}^{N}\right)$ que é isomorfo a $\mathcal{P}_{T}^{\prime}\left(\mathbb{R}^{N}\right)$, na categoria de espaços vetoriais topológicos, considerando em ambos os espaços a topologia fraca* ou considerando a topologia forte em ambos. Para estabelecer tal isomorfismo utilizaremos composição de distribuições com difeomorfismos.

Sejam $\Omega$ e $\Omega^{\prime}$ subconjuntos abertos do $\mathbb{R}^{N}$ e $g: \Omega \rightarrow \Omega^{\prime}$ um difeomorfismo entre eles. Dada uma distribuição $u \in \mathcal{D}^{\prime}\left(\Omega^{\prime}\right)$, definimos a composta $u \circ g \in \mathcal{D}^{\prime}(\Omega)$ por $\langle u \circ g, \phi\rangle \doteq\left\langle u,\left|\operatorname{det} g^{\prime}\right|^{-1} \phi \circ g^{-1}\right\rangle$. No caso em que $g$ é uma translação $g(x)=x-x_{0}$, denotamos a composição $u \circ g$ por $u\left(x-x_{0}\right)$.

Analogamente podemos definir a translação $v\left(x-x_{0}\right)$ de distribuições em $\mathcal{P}_{T}^{\prime}\left(\mathbb{R}^{N}\right)$.

Será demonstrado que o subespaço das distribuições periódicas $\mathcal{D}_{T}^{\prime}\left(\mathbb{R}^{N}\right) \subset \mathcal{D}^{\prime}\left(\mathbb{R}^{N}\right)$ é isomorfo a $\mathcal{P}_{T}^{\prime}\left(\mathbb{R}^{N}\right)$, onde $\mathcal{D}_{T}^{\prime}\left(\mathbb{R}^{N}\right)$ é formado pelas distribuições $u \in \mathcal{D}^{\prime}\left(\mathbb{R}^{N}\right)$ que satisfazem $u=u(x-T m)$, para todo $m \in \mathbb{Z}^{N}$.

Defina a aplicação $H: \mathcal{C}_{c}^{\infty}\left(\mathbb{R}^{N}\right) \rightarrow \mathcal{P}_{T}\left(\mathbb{R}^{N}\right)$ por $H(\phi)(x)=\sum_{m \in \mathbb{Z}^{N}} \phi(x-T m)$, para toda $\phi \in \mathcal{C}_{c}^{\infty}\left(\mathbb{R}^{N}\right)$. Dos comentários feitos no final da seção anterior segue que $H$ está bem definida. Mais ainda, $H$ é uma transformação linear contínua, conforme Proposição (1) da página 4 de [18] e Observação (2.4).

Fixando $\xi \in \mathcal{U}_{T}\left(\mathbb{R}^{N}\right)$ (ver página 31 ), podemos definir $\tilde{H}: \mathcal{P}_{T}\left(\mathbb{R}^{N}\right) \rightarrow \mathcal{C}_{c}^{\infty}\left(\mathbb{R}^{N}\right)$ por $\tilde{H}(\theta)=\theta \xi$, para toda $\theta \in \mathcal{P}_{T}\left(\mathbb{R}^{N}\right)$. Segue da Proposição (2) da página 8 de [18] que $\tilde{H}$ é uma transformação linear contínua.

Como estamos considerando a topologia fraca* ou a topologia forte em ambos os duais $\mathcal{P}_{T}^{\prime}\left(\mathbb{R}^{N}\right)$ e $\mathcal{D}^{\prime}\left(\mathbb{R}^{N}\right)$, segue que os transpostos ${ }^{t} H: \mathcal{P}_{T}^{\prime}\left(\mathbb{R}^{N}\right) \rightarrow \mathcal{D}^{\prime}\left(\mathbb{R}^{N}\right)$ e ${ }^{t} \tilde{H}: \mathcal{D}^{\prime}\left(\mathbb{R}^{N}\right) \rightarrow \mathcal{P}_{T}^{\prime}\left(\mathbb{R}^{N}\right)$ são transformações lineares contínuas, conforme o corolário da Proposição (19.5) de [17]. A próxima proposição assegura que a restrição do operador transposto ${ }^{t} \tilde{H}$ ao subespaço $\mathcal{D}_{T}^{\prime}\left(\mathbb{R}^{N}\right)$ independe da função $\xi \in \mathcal{U}_{T}\left(\mathbb{R}^{N}\right)$.

2.15 Proposição. Sejam $\xi, \eta \in \mathcal{U}_{T}\left(\mathbb{R}^{N}\right)$ e seja $u \in \mathcal{D}_{T}^{\prime}\left(\mathbb{R}^{N}\right)$. Então $\langle u, \xi \theta\rangle=\langle u, \eta \theta\rangle$, para toda $\theta \in \mathcal{P}_{T}\left(\mathbb{R}^{N}\right)$. 
Prova: Se $\sigma \in \mathcal{U}_{T}\left(\mathbb{R}^{N}\right)$, observemos inicialmente que a sequência $\sum_{|m| \leq k}(\sigma u)(x-T m)$ converge para $u$ em $\mathcal{D}^{\prime}\left(\mathbb{R}^{N}\right)$. De fato, como $u \in \mathcal{D}_{T}^{\prime}\left(\mathbb{R}^{N}\right)$, temos

$$
\left\langle u-\sum_{|m| \leq k}(\sigma u)(x-T m), \phi\right\rangle=\left\langle u, \phi(x)-\sum_{|m| \leq k} \phi(x) \sigma(x-T m)\right\rangle,
$$

para cada $\phi \in \mathcal{C}_{c}^{\infty}\left(\mathbb{R}^{N}\right)$. Agora, $\sigma \in \mathcal{U}_{T}\left(\mathbb{R}^{N}\right)$ implica que $\sum_{|m| \leq k} \phi(x) \sigma(x-T m)=\phi(x)$, $x \in \mathbb{R}^{N}$, para $k$ suficientemente grande, logo $\sum_{|m| \leq k} \phi(x) \sigma(x-T m)$ converge para $\phi$ em $\mathcal{C}_{c}^{\infty}\left(\mathbb{R}^{N}\right)$. Assim, $\left\langle u-\sum_{|m| \leq k}(\sigma u)(x-T m), \phi\right\rangle$ converge para zero em $\mathbb{C}$. Por fim, se $\xi, \eta \in \mathcal{U}_{T}\left(\mathbb{R}^{N}\right)$, então

$$
\begin{gathered}
\langle u, \xi \theta\rangle=\lim _{k \rightarrow \infty}\left\langle\sum_{|m| \leq k}(\eta u)(x-T m), \xi \theta\right\rangle=\lim _{k \rightarrow \infty} \sum_{|m| \leq k}\langle(\eta u)(x-T m), \xi \theta\rangle= \\
\lim _{k \rightarrow \infty} \sum_{|m| \leq k}\langle u, \eta(x) \theta(x) \xi(x+T m)\rangle=\lim _{k \rightarrow \infty} \sum_{|m| \leq k}\langle\xi(x+T m) u, \eta \theta\rangle= \\
\lim _{k \rightarrow \infty} \sum_{|m| \leq k}\langle(\xi u)(x+T m), \eta \theta\rangle=\langle u, \eta \theta\rangle,
\end{gathered}
$$

para toda $\theta \in \mathcal{P}_{T}\left(\mathbb{R}^{N}\right)$.

É imediato verificar que ${ }^{t} H\left(\mathcal{P}_{T}^{\prime}\left(\mathbb{R}^{N}\right)\right) \subset \mathcal{D}_{T}^{\prime}\left(\mathbb{R}^{N}\right)$ e que ${ }^{t} \tilde{H} \circ{ }^{t} H$ é o operador identidade de $\mathcal{P}_{T}^{\prime}\left(\mathbb{R}^{N}\right)$.

$\mathrm{Na}$ verdade ${ }^{t} H$ é um isomorfismo entre os espaços vetoriais topológicos $\mathcal{P}_{T}^{\prime}\left(\mathbb{R}^{N}\right)$ e $\mathcal{D}_{T}^{\prime}\left(\mathbb{R}^{N}\right)$ (considerando em ambos a topologia fraca* ou considerando em ambos a topologia forte), com isomorfismo inverso dado por ${ }^{t} \tilde{H}$. Tal afirmação será provada com o próximo teorema.

O produto de uma função $f \in \mathcal{C}^{\infty}(\Omega)$ por uma distribuição $u \in \mathcal{D}^{\prime}(\Omega)$ é a distribuição $f u \in \mathcal{D}^{\prime}(\Omega)$, dada por $\langle f u, \phi\rangle=\langle u, f \phi\rangle$, para toda $\phi \in \mathcal{C}_{c}^{\infty}(\Omega)$. Analogamente, define-se o produto de uma função $f \in \mathcal{P}_{T}\left(\mathbb{R}^{N}\right)$ por uma distribuição $v \in \mathcal{P}_{T}^{\prime}\left(\mathbb{R}^{N}\right)$.

2.16 Teorema. O operador ${ }^{t} H \circ{ }^{t} \tilde{H}$ é o operador identidade de $\mathcal{D}_{T}^{\prime}\left(\mathbb{R}^{N}\right)$.

Prova: Para cada $u \in \mathcal{D}_{T}^{\prime}\left(\mathbb{R}^{N}\right)$ e $\phi \in \mathcal{C}_{c}^{\infty}\left(\mathbb{R}^{N}\right)$, temos

$$
\left\langle\left({ }^{t} H \circ{ }^{t} \tilde{H}\right)(u), \phi\right\rangle=\left\langle{ }^{t} \tilde{H}(u), \sum_{m \in \mathbb{Z}^{N}} \phi(x-T m)\right\rangle=\left\langle u, \xi(x) \sum_{m \in \mathbb{Z}^{N}} \phi(x-T m)\right\rangle .
$$


Utilizando que o suporte de $\xi$ é compacto e que a função $\sum_{m \in \mathbb{Z}^{N}} \phi(x-T m)$ se escreve como uma soma finita em cada bola fechada, verifica-se que a sequência $\xi(x) \sum_{|m| \leq j} \phi(x-T m)$ converge para a função $\xi(x) \sum_{m \in \mathbb{Z}^{N}} \phi(x-T m)$ em $\mathcal{C}_{c}^{\infty}\left(\mathbb{R}^{N}\right)$ e, portanto,

$$
\left\langle u, \xi(x) \sum_{m \in \mathbb{Z}^{N}} \phi(x-T m)\right\rangle=\lim _{j \rightarrow \infty}\left\langle u, \xi(x) \sum_{|m| \leq j} \phi(x-T m)\right\rangle .
$$

Como $u \in \mathcal{D}_{T}^{\prime}\left(\mathbb{R}^{N}\right)$, temos

$$
\left\langle u, \xi(x) \sum_{|m| \leq j} \phi(x-T m)\right\rangle=\left\langle u, \phi(x) \sum_{|m| \leq j} \xi(x+T m)\right\rangle .
$$

Como $\xi \in \mathcal{U}_{T}\left(\mathbb{R}^{N}\right)$, temos $\phi(x) \sum_{|m| \leq j} \xi(x+T m)=\phi(x)$ em $\mathbb{R}^{N}$, para $j$ suficientemente grande, logo $\phi(x) \sum_{|m| \leq j} \xi(x+T m)$ converge para $\phi$ em $\mathcal{C}_{c}^{\infty}\left(\mathbb{R}^{N}\right)$. Então

$$
\lim _{j \rightarrow \infty}\left\langle u, \phi(x) \sum_{|m| \leq j} \xi(x+T m)\right\rangle=\langle u, \phi\rangle
$$

e, consequentemente, $\left\langle\left({ }^{t} H \circ{ }^{t} \tilde{H}\right)(u), \phi\right\rangle=\langle u, \phi\rangle$.

A próxima definição trata da noção de suporte no contexto de distribuições. Se $\Omega$ e $\Omega^{\prime}$ são subconjuntos abertos do $\mathbb{R}^{N}$ com $\Omega^{\prime} \subset \Omega$, então podemos restringir uma distribuição $u \in \mathcal{D}^{\prime}(\Omega)$ a uma distribuição $v \in \mathcal{D}^{\prime}\left(\Omega^{\prime}\right)$, definindo $\langle v, \phi\rangle \doteq\langle u, \phi\rangle$, para toda $\phi \in \mathcal{C}_{c}^{\infty}\left(\Omega^{\prime}\right)$.

Dizer que duas distribuições $u$ e $v \in \mathcal{D}^{\prime}(\Omega)$ coincidem significa que $\langle u, \phi\rangle=\langle v, \phi\rangle$, para toda $\phi \in \mathcal{C}_{c}^{\infty}(\Omega)$, afinal, $u$ e $v$ são funcionais lineares.

Existe também um conceito de localização que determina uma distribuição em $\mathcal{D}^{\prime}(\Omega)$; a saber, $u$ e $v \in \mathcal{D}^{\prime}(\Omega)$ coincidem se, e somente se, suas restrições a qualquer subconjunto aberto de $\Omega$ coincidem. Tal resultado é uma consequência imediata do seguinte lema.

2.17 Lema. Se $u \in \mathcal{D}^{\prime}(\Omega)$ e cada ponto de $\Omega$ possui uma vizinhança aberta restrita a qual u é a distribuição nula, então $u=0$ em $\mathcal{D}^{\prime}(\Omega)$.

A demonstração do lema acima pode ser consultada nas referências [11] ou [12]. 
2.18 Definição. O suporte de uma distribuição $u \in \mathcal{D}^{\prime}(\Omega), S(u)$, é a interseção de todos os fechados de $\Omega$ fora dos quais u é nula. Equivalentemente, $S(u)$ é o conjunto dos pontos de $\Omega$ que não possuem uma vizinhança restrita a qual u é nula.

Denotaremos por $\mathcal{E}^{\prime}(\Omega)$ o subespaço de $\mathcal{D}^{\prime}(\Omega)$ das distribuições que possuem suporte compacto. O teorema abaixo garante que cada distribuição $u \in \mathcal{E}^{\prime}(\Omega)$ se estende a um único funcional linear $\tilde{u}$ definido em $\mathcal{C}^{\infty}(\Omega)$. Sua demonstraçao pode ser consultada na página 37 da referência [12].

2.19 Teorema. Para cada $u \in \mathcal{E}^{\prime}(\Omega)$, existe um único funcional linear $\tilde{u}: \mathcal{C}^{\infty}(\Omega) \rightarrow \mathbb{C}$ que satisfaz

(i) $\langle\tilde{u}, \phi\rangle=\langle u, \phi\rangle$, para toda $\phi \in \mathcal{C}_{c}^{\infty}(\Omega)$.

(ii) $\langle\tilde{u}, \phi\rangle=0$, se $\phi \in \mathcal{C}^{\infty}(\Omega)$ e $S(\phi) \cap S(u)=\emptyset$.

2.20 Observação. Não adotaremos a notação $\tilde{u}$, cometeremos um abuso de notação e continuaremos a denotar por $u$ a extensão de $u \in \mathcal{E}^{\prime}(\Omega)$. Assim, $\langle u, \phi\rangle$ fica definido para cada $u \in \mathcal{E}^{\prime}(\Omega)$ e $\phi \in \mathcal{C}^{\infty}(\Omega)$.

Podemos também derivar distribuições em $\mathcal{D}^{\prime}(\Omega)$ e $\mathcal{P}_{T}^{\prime}\left(\mathbb{R}^{N}\right)$ no seguinte sentido. Para cada $\alpha \in \mathbb{Z}_{+}^{N}$, definimos $\partial^{\alpha} u$ por $\left\langle\partial^{\alpha} u, \phi\right\rangle=(-1)^{|\alpha|}\left\langle u, \partial^{|\alpha|} \phi\right\rangle$.

A proposição abaixo destaca algumas propriedades dos isomorfismos ${ }^{t} H$ e ${ }^{t} \tilde{H}$.

2.21 Proposição. Os isomorfismos ${ }^{t} H e{ }^{t} \tilde{H}$ possuem as seguintes propriedades:

(i) ${ }^{t} H(f v)=f^{t} H(v) e{ }^{t} \tilde{H}(f u)=f{ }^{t} \tilde{H}(u)$, para quaisquer $f \in \mathcal{P}_{T}\left(\mathbb{R}^{N}\right), v \in \mathcal{P}_{T}^{\prime}\left(\mathbb{R}^{N}\right)$ e $u \in \mathcal{D}_{T}^{\prime}\left(\mathbb{R}^{N}\right)$.

(ii) ${ }^{t} H\left(\partial^{\alpha} v\right)=\partial^{\alpha}\left({ }^{t} H(v)\right) e{ }^{t} \tilde{H}\left(\partial^{\alpha} u\right)=\partial^{\alpha}\left({ }^{t} \tilde{H}(u)\right)$, quaisquer que sejam $v \in \mathcal{P}_{T}^{\prime}\left(\mathbb{R}^{N}\right)$, $u \in \mathcal{D}_{T}^{\prime}\left(\mathbb{R}^{N}\right)$ e $\alpha \in \mathbb{Z}_{+}^{N}$.

(iii) ${ }^{t} H\left(v\left(x-x_{0}\right)\right)={ }^{t} H(v)\left(x-x_{0}\right) e{ }^{t} \tilde{H}\left(u\left(x-x_{0}\right)\right)={ }^{t} \tilde{H}(u)\left(x-x_{0}\right)$, para quaisquer $x_{0} \in \mathbb{R}^{N}, v \in \mathcal{P}_{T}^{\prime}\left(\mathbb{R}^{N}\right)$ e $u \in \mathcal{D}_{T}^{\prime}\left(\mathbb{R}^{N}\right)$.

(iv) ${ }^{t} H 1=1 e^{t} \tilde{H} 1=1$.

O próximo teorema é uma espécie de lema da colagem para distribuições.

2.22 Teorema. Seja $\left\{\Omega_{j}\right\}_{j \in J}$ uma familia de abertos do $\mathbb{R}^{N}, \Omega=\bigcup_{j \in J} \Omega_{j}$ e $\left\{u_{j}\right\}_{j \in J}$ uma família de distribuições satisfazendo $u_{j} \in \mathcal{D}^{\prime}\left(\Omega_{j}\right)$ e $u_{j}=u_{l}$ em $\Omega_{j} \cap \Omega_{l}$, para cada $j, l \in J$. Então existe uma única distribuição $u \in \mathcal{D}^{\prime}(\Omega)$ cuja restrição a $\Omega_{j}$ é $u_{j}$, para cada $j \in J$. 
Prova: A unicidade segue diretamente do Lema (2.17).

De acordo com o Teorema (2.6), podemos escrever cada função $\phi \in \mathcal{C}_{c}^{\infty}(\Omega)$ como uma soma $\phi=\sum_{j \in J} \phi_{j}$, onde $\phi_{j} \in \mathcal{C}_{c}^{\infty}\left(\Omega_{j}\right)$ e $\phi_{j}$ não é identicamente nula apenas para um número finito de índices. Assim, definimos

$$
u(\phi)=\sum_{j \in J}\left\langle u_{j}, \phi_{j}\right\rangle
$$

Para mostrar que $u$ está bem definida basta tomar $\sum_{j \in J} \phi_{j}=0, \operatorname{com} \phi_{j} \in \mathcal{C}_{c}^{\infty}\left(\Omega_{j}\right)$ e $\phi_{j}$ não identicamente nula apenas para um número finito de índices, e verificar que $\sum_{j \in J}\left\langle u_{j}, \phi_{j}\right\rangle=0$

Denote por $K$ a reunião dos suportes das funções $\phi_{j}$. Como $K$ é um compacto, pois é uma reunião finita de compactos, utilizamos novamente o Teorema (2.6) para obter funções $\psi_{j} \in \mathcal{C}_{c}^{\infty}\left(\Omega_{j}\right)$ satisfazendo $\sum_{j \in J} \psi_{j} \equiv 1$ em $K$, com $\psi_{j}$ não identicamente nula apenas para um número finito de índices.

Para cada $j, l \in J$, temos $\phi_{j} \psi_{l} \in \mathcal{C}_{c}^{\infty}\left(\Omega_{j} \cap \Omega_{l}\right)$ e assim

$$
\begin{gathered}
\sum_{j \in J}\left\langle u_{j}, \phi_{j}\right\rangle=\sum_{j \in J}\left\langle u_{j}, \sum_{l \in J} \psi_{l} \phi_{j}\right\rangle=\sum_{j \in J} \sum_{l \in J}\left\langle u_{j}, \psi_{l} \phi_{j}\right\rangle=\sum_{j \in J} \sum_{l \in J}\left\langle u_{l}, \psi_{l} \phi_{j}\right\rangle= \\
\sum_{l \in J}\left\langle u_{l}, \psi_{l} \sum_{j \in J} \phi_{j}\right\rangle=0 .
\end{gathered}
$$

Direto da definição segue que $u$ é um funcional linear definido em $\mathcal{C}_{c}^{\infty}(\Omega)$; sendo assim, resta apenas provarmos a continuidade de $u$.

Seja $K^{\prime} \subset \Omega$ um compacto. Tome $\psi_{j} \in \mathcal{C}_{c}^{\infty}\left(\Omega_{j}\right), j \in J$, satisfazendo $\sum_{j \in J} \psi_{j} \equiv 1 \mathrm{em}$ $K^{\prime}$, com $\psi_{j}$ não identicamente nula apenas para um número finito de índices $j_{1}, \ldots, j_{n}$. Para cada $\phi \in \mathcal{C}_{c}^{\infty}(\Omega) \operatorname{com} S(\phi) \subset K^{\prime}$, temos

$$
\begin{gathered}
|\langle u, \phi\rangle|=\left|\left\langle u, \sum_{j \in J} \phi \psi_{j}\right\rangle\right| \leq \sum_{i=1}^{n}\left|\left\langle u_{j_{i}}, \phi \psi_{j_{i}}\right\rangle\right| \leq \sum_{i=1}^{n} C_{i} \sum_{|\alpha| \leq m_{i}} \sup \left|\partial^{\alpha}\left(\phi \psi_{j_{i}}\right)\right| \leq \\
C \sum_{|\alpha| \leq m=\max \left\{m_{i}\right\}} \sup \left|\partial^{\alpha}(\phi)\right|,
\end{gathered}
$$

onde cada $m_{i}$ é um natural, cada $C_{i}$ é um real positivo e $C$ é uma constante que não depende de $\phi$, o que mostra que $u \in \mathcal{D}^{\prime}(\Omega)$ é uma distribuição. 
2.23 Observação. Nas condições do teorema anterior, se cada $u_{j}$ for de ordem finita e existir $M$, um limitante superior para as ordens das distribuições $u_{j}$, então a distribuição $u$ será de ordem finita menor ou igual a $M$. Mais ainda, se cada $u_{j}$ for de ordem finita e existir $M$, o máximo das ordens das distribuições $u_{j}$, então a distribuição $u$ será de ordem finita igual a $M$. Tal afirmação pode ser verificada analisando-se a parte final da demonstração anterior.

Considere a distribuição $c \partial^{\alpha} \delta\left(x-x_{0}\right) \in \mathcal{D}^{\prime}(\Omega)$, onde $c \in \mathbb{C} \backslash\{0\}, \alpha \in \mathbb{Z}_{+}^{N}$ e $x_{0} \in \Omega$. Diretamente da Definição (2.18) verifica-se que $S\left(c \partial^{\alpha} \delta\left(x-x_{0}\right)\right) \subset\left\{x_{0}\right\}$ e utilizando uma função do tipo $\phi(x)=\psi(x)\left(x-x_{0}\right)^{\alpha}$, onde $\psi$ é uma função de corte, isto é, $\psi \in \mathcal{C}_{c}^{\infty}(\Omega)$ e $\psi \equiv 1$ em uma vizinhança de $\left\{x_{0}\right\}$, obtemos $S\left(c \partial^{\alpha} \delta\left(x-x_{0}\right)\right)=\left\{x_{0}\right\}$. Mais geralmete, se $u=\sum_{|\alpha| \leq m} c_{\alpha} \partial^{\alpha} \delta\left(x-x_{0}\right)$, onde $m \in \mathbb{Z}_{+}$e $c_{\alpha} \in \mathbb{C}$, então $S(u) \subset\left\{x_{0}\right\}$. O interessante é que a recíproca desse resultado é verdadeira, conforme veremos no próximo teorema.

Vale deixar registrado que utilizando funções como $\phi$, temos $0=\sum_{|\alpha| \leq m} c_{\alpha} \partial^{\alpha} \delta\left(x-x_{0}\right)$ se, e somente se, $c_{\alpha}=0$ para todo $\alpha$. Consequentemente, $S(u)=\left\{x_{0}\right\}$ se, e somente se, $c_{\alpha} \neq 0$, para algum $\alpha$.

2.24 Teorema. Se $x_{0} \in \Omega \subset \mathbb{R}^{N}$ e $u \in \mathcal{D}^{\prime}(\Omega)$ satisfaz $S(u)=\left\{x_{0}\right\}$, então

$$
u=\sum_{|\alpha| \leq m} c_{\alpha} \partial^{\alpha} \delta\left(x-x_{0}\right)
$$

com $c_{\alpha} \in \mathbb{C}$, para todo $|\alpha| \leq m$. Mais ainda, o natural $m$ pode ser tomado igual à ordem da distribuição $u$.

Prova: Suponha inicialmente que $x_{0}=0 \in \Omega$. Tome $\epsilon>0$ de forma que $B_{3 \epsilon}=$ $B(0,3 \epsilon) \subset B[0,3 \epsilon] \subset \Omega$ e escolha $\phi \in \mathcal{C}_{c}^{\infty}\left(\mathbb{R}^{N}\right)$ satisfazendo $S(\phi) \subset B_{1}=B(0,1), \phi \geq 0$ e $\int_{B_{1}} \phi=1$.

Defina

$$
\phi_{\epsilon}(x)=\epsilon^{-N} \int_{B_{2 \epsilon}} \phi\left(\frac{x-y}{\epsilon}\right) d y,
$$

para cada $x \in \mathbb{R}^{N}$. Então $\phi_{\epsilon} \in \mathcal{C}_{c}^{\infty}\left(B_{3 \epsilon}\right)$ e $\phi_{\epsilon} \equiv 1$ em $B_{\epsilon}$.

Do Teorema (2.19) segue que $\langle u, \psi\rangle=\left\langle u, \phi_{\epsilon} \psi\right\rangle$, para cada $\psi \in \mathcal{C}^{\infty}(\Omega)$, pois $u \in \mathcal{E}^{\prime}(\Omega)$ e $S\left(\psi\left(1-\phi_{\epsilon}\right)\right) \cap(\{0\}=S(u))=\emptyset$.

Escolha $r>0$ tal que $B[0, r] \subset \Omega$. Segue da continuidade de $u$ que existem $C>0$ e $m \in \mathbb{N}$ tal que

$$
|\langle u, \psi\rangle| \leq C \sum_{|\alpha| \leq m} \sup _{x \in \Omega}\left|\partial^{\alpha}\left(\phi_{\epsilon} \psi\right)\right|
$$


para todo $0<\epsilon<r / 3$ e $\psi \in \mathcal{C}^{\infty}(\Omega)$, uma vez que $S\left(\phi_{\epsilon}\right) \subset B[0, r]$, se $0<\epsilon<r / 3$. Como $u \in \mathcal{E}^{\prime}(\Omega), u$ é uma distribuição de ordem finita e podemos tomar $m$ igual à ordem de $u$.

Para $\psi \in \mathcal{C}_{c}^{\infty}(\Omega)$, o desenvolvimento de Taylor de $\psi$ em torno da origem fornece $\psi(x)=\sum_{|\alpha| \leq m} \partial^{\alpha} \psi(0) x^{\alpha} / \alpha !+\left(R_{m+1}(x) \doteq(m+1) \int_{0}^{1}(1-t)^{m} \sum_{|\alpha|=m+1} \partial^{\alpha} \psi(t x) x^{\alpha} / \alpha ! d t\right)$

para todo $x \in B[0, r]$. Utilizando a fórmula de Leibniz e derivação sobre o sinal de integração, verifica-se que

$$
\left|\partial^{\beta} R_{m+1}(x)\right| \leq M|x|^{m+1-|\beta|}
$$

para todo $x \in B[0, r]$ e $|\beta| \leq m+1$, onde $M$ é uma constante que depende de $\psi, m$ e $\beta$, mas não depende de $x \in B[0, r]$. Assim,

$$
\langle u, \psi\rangle=\sum_{|\alpha| \leq m} \partial^{\alpha} \psi(0)\left\langle u, x^{\alpha}\right\rangle / \alpha !+\left\langle u, R_{m+1}\right\rangle=\left\langle\sum_{|\alpha| \leq m} c_{\alpha} \partial^{\alpha} \delta, \psi\right\rangle+\left\langle u, R_{m+1}\right\rangle
$$

Note que o Teorema (2.19) garante que $\left\langle u, x^{\alpha}\right\rangle$ e $\left\langle u, R_{m+1}\right\rangle$ estão bem definidos. Para finalizar a demonstração deste caso basta provarmos que $\left\langle u, R_{m+1}\right\rangle=0$. Segue da desigualdade (.1) que

$$
\left|\left\langle u, R_{m+1}\right\rangle\right| \leq C \sum_{|\alpha| \leq m} \sup _{x \in \Omega}\left|\partial^{\alpha}\left(\phi_{\epsilon} R_{m+1}\right)(x)\right|=C \sum_{|\alpha| \leq m} \sup _{x \in B_{3 \epsilon}}\left|\partial^{\alpha}\left(\phi_{\epsilon} R_{m+1}\right)(x)\right|,
$$

para todo $0<\epsilon<r / 3$. Utilizando a definição de $\phi_{\epsilon}$ verifica-se que $\sup _{x \in B_{3 \epsilon}}\left|\partial^{\beta} \phi_{\epsilon}(x)\right| \leq$ $\epsilon^{-|\beta|} L_{\beta}$, onde $L_{\beta}$ é uma constante que depende de $\beta \in \mathbb{Z}_{+}^{N}$ e não depende de $\epsilon$.

Pela fórmula de Leibniz e pela desigualdade (.2), temos

$$
\begin{gathered}
\left|\left\langle u, R_{m+1}\right\rangle\right| \leq C \sum_{|\alpha| \leq m} \alpha ! \sum_{\beta \leq \alpha} \sup _{x \in B_{3 \epsilon}}\left|\partial^{\beta} \phi_{\epsilon}(x)\right| . \sup _{x \in B_{3 \epsilon}}\left|\partial^{\alpha-\beta} R_{m+1}(x)\right| \leq \\
C \sum_{|\alpha| \leq m} \alpha ! \sum_{\beta \leq \alpha} \epsilon^{-|\beta|} L_{\beta} M(3 \epsilon)^{m+1-|\alpha-\beta|}=C \sum_{|\alpha| \leq m} \alpha ! \sum_{\beta \leq \alpha} L_{\beta} M 3^{m+1-|\alpha-\beta|} \epsilon^{m+1-|\alpha|} \leq \\
\left(C \sum_{|\alpha| \leq m} \alpha ! \sum_{\beta \leq \alpha} L_{\beta} M 3^{m+1-|\alpha-\beta|}\right) \epsilon=\tilde{C} \epsilon,
\end{gathered}
$$


para todo $\epsilon \in(0, \min \{1, r / 3\})$; onde $\tilde{C}$ é uma constante que não depende de $\epsilon$. Assim, obtemos $\left|\left\langle u, R_{m+1}\right\rangle\right|=0$.

No caso geral em que $x_{0} \in \Omega$, compondo a distribuição $u$ com uma translação obtemos $u\left(x+x_{0}\right) \in \mathcal{D}^{\prime}\left(\Omega-x_{0}\right)$ com $S\left(u\left(x-x_{0}\right)\right)=\{0\}$. Segue do caso anterior que $u\left(x+x_{0}\right)=\sum_{|\alpha| \leq m} c_{\alpha} \partial^{\alpha} \delta$ em $\mathcal{D}^{\prime}\left(\Omega-x_{0}\right)$ e compondo ambos os lados da igualdade com a translação inversa, obtemos $u=\sum_{|\alpha| \leq m} c_{\alpha} \partial^{\alpha} \delta\left(x-x_{0}\right)$ em $\mathcal{D}^{\prime}(\Omega)$, o que finaliza a demonstração do teorema.

O teorema anterior fornece que alguns operadores não modificam a forma de uma distribuição que é escrita como combinação linear de derivadas da delta. Por exemplo, sejam $x_{0} \in I \subset \mathbb{R}, k \in \mathbb{Z}$ e $f \in \mathcal{C}^{\infty}(I)$. Se $u \in \mathcal{D}^{\prime}(I)$ for uma combinação linear de derivadas de $\delta\left(x-x_{0}\right)$, então $w=(f u)^{\prime}+i k u \in \mathcal{D}^{\prime}(I)$ também será uma combinação linear de derivadas de $\delta\left(x-x_{0}\right)$, uma vez que $S(w) \subset S(u) \subset\left\{x_{0}\right\}$. Uma pergunta que pode ser feita é saber se para cada $w \in \mathcal{D}^{\prime}(I)$ que for uma combinação linear de derivadas de $\delta\left(x-x_{0}\right)$, existirá $u \in \mathcal{D}^{\prime}(I)$ tal que $\mathrm{P}_{k}(u) \doteq(f u)^{\prime}+i k u=w$.

2.25 Proposição. Sejam $x_{0} \in I \subset \mathbb{R}, k \in \mathbb{Z} \backslash\{0\}, f \in \mathcal{C}^{\infty}(I, \mathbb{R})$ tal que $x_{0} \in f^{-1}(0)$ e $\mathrm{P}_{k} \doteq d / d x(f \cdot)+(i k \cdot) . S e$

$$
w=\sum_{j=0}^{r} c_{j} \partial^{j} \delta\left(x-x_{0}\right) \in \mathcal{D}^{\prime}(I)
$$

então existe

$$
u=\sum_{j=0}^{r} b_{j} \partial^{j} \delta\left(x-x_{0}\right) \in \mathcal{D}^{\prime}(I)
$$

tal que $\mathrm{P}_{k}(u)=(f u)^{\prime}+i k u=w$.

Prova: Fazendo $j=0$, temos $f \delta\left(x-x_{0}\right)=0$ e para $j \geq 1$, temos $f \partial^{j} \delta\left(x-x_{0}\right)=$ $\sum_{l=0}^{j}\left(\begin{array}{l}j \\ l\end{array}\right)(-1)^{l}\left(\partial^{l} f\right)\left(x_{0}\right) \partial^{j-l} \delta\left(x-x_{0}\right)=\sum_{l=1}^{j}\left(\begin{array}{l}j \\ l\end{array}\right)(-1)^{l}\left(\partial^{l} f\right)\left(x_{0}\right) \partial^{j-l} \delta\left(x-x_{0}\right)$. Assim, para $j \geq 1$, temos $\left(f \partial^{j} \delta\left(x-x_{0}\right)\right)^{\prime}=\sum_{l=1}^{j}\left(\begin{array}{l}j \\ l\end{array}\right)(-1)^{l}\left(\partial^{l} f\right)\left(x_{0}\right) \partial^{j-l+1} \delta\left(x-x_{0}\right)$. Portanto, para

$$
u=\sum_{j=0}^{r} b_{j} \partial^{j} \delta\left(x-x_{0}\right) \in \mathcal{D}^{\prime}(I)
$$

temos

$$
\mathrm{P}_{k} u=\sum_{j=0}^{r} b_{j}\left(f \partial^{j} \delta\left(x-x_{0}\right)\right)^{\prime}+i k \sum_{j=0}^{r} b_{j} \partial^{j} \delta\left(x-x_{0}\right)=
$$




$$
\begin{aligned}
& \sum_{j=1}^{r} b_{j} \sum_{l=1}^{j}\left(\begin{array}{l}
j \\
l
\end{array}\right)(-1)^{l}\left(\partial^{l} f\right)\left(x_{0}\right) \partial^{j-l+1} \delta\left(x-x_{0}\right)+i k \sum_{j=0}^{r} b_{j} \partial^{j} \delta\left(x-x_{0}\right)= \\
& \sum_{j=1}^{r}\left(\sum_{l=j}^{r} b_{l} C_{l, l-j+1}\left(\partial^{l-j+1} f\right)\left(x_{0}\right)\right) \partial^{j} \delta\left(x-x_{0}\right)+i k \sum_{j=0}^{r} b_{j} \partial^{j} \delta\left(x-x_{0}\right),
\end{aligned}
$$

onde $C_{j, l} \doteq\left(\begin{array}{l}j \\ l\end{array}\right)(-1)^{l} \in \mathbb{R}$, para $1 \leq j \leq r$ e $1 \leq l \leq r$. Portanto, $\mathrm{P}_{k} u=w$ se, e somente se, $i k b_{0}=c_{0} \mathrm{e}$

$$
i k b_{j}+\sum_{l=j}^{r} b_{l} C_{l, l-j+1}\left(\partial^{l-j+1} f\right)\left(x_{0}\right)=c_{j}
$$

para $j=1, \ldots, r$, ou seja, $i k b_{0}=c_{0},\left[i k+C_{r, 1} f^{\prime}\left(x_{0}\right)\right] b_{r}=c_{r}$ e

$$
\left[i k+C_{j, 1} f^{\prime}\left(x_{0}\right)\right] b_{j}=c_{j}-\sum_{l=j+1}^{r} b_{l} C_{l, l-j+1}\left(\partial^{l-j+1} f\right)\left(x_{0}\right),
$$

para $j=1, \ldots, r-1$. Como as constantes $C_{j, l}$ são reais e a função $f$ é uma função real, utilizamos indução para obter, de maneira única, $b_{0}, b_{r}, b_{r-1}, \ldots, b_{1}$ tais que $u=$ $\sum_{j=0}^{r} b_{j} \partial^{j} \delta\left(x-x_{0}\right)$ satisfaz $\mathrm{P}_{k} u=w$.

Também vale um resultado análogo à proposição anterior para uma outra classe de operadores. A demonstração é bem semelhante à demonstração anterior.

2.26 Proposição. Sejam $x_{0} \in I \subset \mathbb{R}, k \in \mathbb{Z} \backslash\{0\}, f \in \mathcal{C}^{\infty}(I, \mathbb{R})$ tal que $x_{0} \in f^{-1}(0)$ e $\mathrm{L}_{k} \doteq f(x) d / d x+(i k \cdot) . S e$

$$
w=\sum_{j=0}^{r} c_{j} \partial^{j} \delta\left(x-x_{0}\right) \in \mathcal{D}^{\prime}(I)
$$

então existe

$$
u=\sum_{j=0}^{r} b_{j} \partial^{j} \delta\left(x-x_{0}\right) \in \mathcal{D}^{\prime}(I)
$$

tal que $\mathrm{L}_{k}(u)=f u^{\prime}+i k u=w$.

Considere $g \in \mathcal{C}^{\infty}(I)$ uma função que se anula apenas em $x_{0} \in I$, o qual é um zero de ordem finita. O próximo resultado caracteriza as soluções de uma equação da forma $g u=0$, onde $u \in \mathcal{D}^{\prime}(I)$. 
2.27 Teorema. Seja $g \in \mathcal{C}^{\infty}(I)$ uma função que se anula apenas em $x_{0} \in I \subset \mathbb{R}, o$ qual é um zero de ordem finita $r \geq 1$. Se $u \in \mathcal{D}^{\prime}(I)$ satisfaz gu $=0$, então

$$
u=\sum_{0 \leq j \leq r-1} c_{j} \partial^{j} \delta\left(x-x_{0}\right)
$$

onde $c_{j} \in \mathbb{C}$, para todo $0 \leq j \leq r-1$.

Prova: Como $g$ se anula apenas em $x_{0}$, temos $S(u) \subset\left\{x_{0}\right\}$. Segue do Teorema $(2.24)$ que

$$
u=\sum_{0 \leq j \leq n} c_{j} \partial^{j} \delta\left(x-x_{0}\right),
$$

onde $n \in \mathbb{Z}_{+}$e $c_{j} \in \mathbb{C}$, para $j=0, \ldots, n$. Supondo que $n \geq r$, obtemos da hipótese $g u=0$ a igualdade

$$
0=\sum_{0 \leq j \leq r-1} c_{j}\left(x-x_{0}\right)^{r} \partial^{j} \delta\left(x-x_{0}\right)+\sum_{r \leq j \leq n} c_{j}\left(x-x_{0}\right)^{r} \partial^{j} \delta\left(x-x_{0}\right) .
$$

Como $\left(x-x_{0}\right)^{r} \partial^{j} \delta\left(x-x_{0}\right)=0$, se $j<r$ e $\left(x-x_{0}\right)^{r} \partial^{j} \delta\left(x-x_{0}\right)=(-1)^{r}\left(\begin{array}{l}j \\ r\end{array}\right) r ! \partial^{j-r} \delta\left(x-x_{0}\right)$, se $j \geq r$, temos

$$
0=\sum_{r \leq j \leq n} c_{j}(-1)^{r}\left(\begin{array}{l}
j \\
r
\end{array}\right) r ! \partial^{j-r} \delta\left(x-x_{0}\right) .
$$

A independência linear entre a distribuição $\delta$ e suas derivadas implica que $c_{j}=0$, para todo $r \leq j \leq n$.

A definição da multiplicação de uma função infinitamente diferenciável por uma distribuição permite elaborar o problema de dividir uma distribuição $u \in \mathcal{D}^{\prime}(\Omega)$ por uma função $f \in \mathcal{C}^{\infty}(\Omega)$, o qual consite em encontrar outra distribuição $v \in \mathcal{D}^{\prime}(\Omega)$ tal que $f v=u$. Se $f^{-1}(0)=\emptyset$, então existe uma única solução, dada por $v=f^{-1} u$.

Um problema de divisão também pode possuir mais do que uma solução, bem como não possuir solução alguma. Por exemplo, dividir a distribuição nula por uma função de $\mathcal{C}^{\infty}(I)$ que se anula apenas em $x_{0} \in I$, o qual é um zero de ordem finita, é um problema em que a solução não é única. O Teorema (2.27) fornece todas as soluções de tal problema de divisão.

Faremos algumas considerações a respeito do problema de divisão em uma variável. Inicialmente, mostraremos que se $f$ possuir apenas zeros de ordem finita, então sempre será possível dividir uma distribuição de $\mathcal{D}^{\prime}(I)$ por $f$. Depois exibiremos algumas distribuições que não podem ser divididas por funções que possuem anulamentos de ordem infinita. 
2.28 Teorema. Considere $I \subset \mathbb{R}$ um intervalo aberto e limitado.

(i) Seja $f \in \mathcal{C}^{\infty}(I)$ uma função que se anula apenas em $x_{0} \in I$, o qual é um zero de ordem finita igual a $n$. Dada $u \in \mathcal{D}^{\prime}(I)$, existe $v \in \mathcal{D}^{\prime}(I)$ tal que $f v=u$. Mais ainda, se u for de ordem finita menor ou igual a $m$, então poderemos tomar $v \in \mathcal{D}^{\prime}(I)$ de ordem finita menor ou igual a $m+n$.

(ii) Seja $f \in \mathcal{P}_{2 \pi}(\mathbb{R})$ uma função que possua apenas zeros de ordem finita. Dada $u \in \mathcal{D}_{2 \pi}^{\prime}(\mathbb{R})$, existe $v \in \mathcal{D}_{2 \pi}^{\prime}(\mathbb{R})$ tal que $f v=u$ Mais ainda, se $u$ for de ordem finita menor ou igual a $m$ e $M$ for um limitante superior para a ordem dos zeros de $f$, então poderemos tomar $v \in \mathcal{D}_{2 \pi}^{\prime}(\mathbb{R})$ de ordem finita menor ou igual a $M+m$.

Prova: (i) Inicialmente demonstraremos o caso em que $f(x)=x-x_{0}$. Tome $\chi \in \mathcal{C}_{c}^{\infty}(I)$, com $0 \leq \chi \leq 1$ e $\chi \equiv 1$ em uma vizinhança de $x_{0}$. Para cada função $\phi \in \mathcal{C}_{c}^{\infty}(I)$, temos $\phi\left(x_{0}+x\right)=\phi\left(x_{0}\right)+\int_{0}^{1} \phi^{\prime}\left(x_{0}+t x\right) x d t$, para todo $x \in I-x_{0}$. Defina $\psi_{\phi}(x)=$ $\int_{0}^{1} \phi^{\prime}\left(x_{0}+t x\right) d t$, para todo $x \in I-x_{0}$. Como $\psi_{\phi}\left(x-x_{0}\right) \in \mathcal{C}^{\infty}(I)$, podemos definir

$$
\langle v, \phi\rangle=\left\langle u, \chi(x) \psi_{\phi}\left(x-x_{0}\right)\right\rangle+\left\langle u,\left(x-x_{0}\right)^{-1}(1-\chi(x)) \phi(x)\right\rangle,
$$

para toda $\phi \in \mathcal{C}_{c}^{\infty}(I)$.

Observe que $v$ é um funcional linear definido em $\mathcal{C}_{c}^{\infty}(I)$. Mais ainda, para cada $\phi \in \mathcal{C}_{c}^{\infty}(I)$, a função $\theta(x)=\left(x-x_{0}\right) \phi(x)$ pertence a $\mathcal{C}_{c}^{\infty}(I)$ e satisfaz $\theta\left(x_{0}\right)=0$; assim, $x \psi_{\theta}(x)=\theta\left(x_{0}+x\right)=x \phi\left(x+x_{0}\right)$ e, consequentemente, $\psi_{\theta}(x)=\phi\left(x+x_{0}\right)$, para todo $x \in I-x_{0}$. Portanto,

$$
\begin{gathered}
\left\langle\left(x-x_{0}\right) v, \phi\right\rangle=\left\langle v,\left(x-x_{0}\right) \phi(x)\right\rangle=\langle v, \theta\rangle=\left\langle u, \chi(x) \psi_{\theta}\left(x-x_{0}\right)\right\rangle+ \\
\left\langle u,\left(x-x_{0}\right)^{-1}(1-\chi(x)) \theta(x)\right\rangle=\langle u, \chi \phi\rangle+\langle u,(1-\chi) \phi\rangle=\langle u, \phi\rangle .
\end{gathered}
$$

Então $v$ é um funcional linear definido em $\mathcal{C}_{c}^{\infty}(I)$ que satisfaz $\left(x-x_{0}\right) v=u$. Resta provarmos a continuidade de $v$.

Fixe $K \subset I$ um compacto. Como $u \in \mathcal{D}^{\prime}(I)$, existem $C>0$ e $m \in \mathbb{Z}_{+}$tais que

$$
|\langle u, \psi\rangle| \leq C \sum_{j=0}^{m} \sup _{x \in I}\left|\partial^{j} \psi(x)\right|,
$$

para toda $\psi \in \mathcal{C}_{c}^{\infty}(I) \operatorname{com} S(\psi) \subset K \cup S(\chi)$. 
Utilizando a fómula de Leibniz, obtemos

$$
\left|\left\langle u, \chi \psi_{\phi}\left(x-x_{0}\right)\right\rangle\right| \leq \tilde{C} \sum_{j=0}^{m} \sup _{x \in I}\left|\partial^{j} \psi_{\phi}\left(x-x_{0}\right)\right|
$$

onde $\tilde{C}>0$ é uma constante que independe de $\phi \in \mathcal{C}_{c}^{\infty}(I)$. Conseguimos obter tal constante uma vez que $\chi$ e todas as suas derivadas são limitadas em $I$. Note que, para cada $0 \leq j \leq m$, temos $\partial^{j} \psi_{\phi}\left(x-x_{0}\right)=\int_{0}^{1} t^{j} \partial^{j+1} \phi\left(x_{0}+t x\right) d t$ e, portanto, $\sup _{x \in I}\left|\partial^{j} \psi_{\phi}\left(x-x_{0}\right)\right| \leq \sup _{y \in I}\left|\partial^{j+1} \phi(y)\right|$. Resulta então que

$$
\left|\left\langle u, \chi \psi_{\phi}\left(x-x_{0}\right)\right\rangle\right| \leq \tilde{C} \sum_{j=0}^{m+1} \sup _{x \in I}\left|\partial^{j} \phi(x)\right|
$$

Como $\left(x-x_{0}\right)^{-1}(1-\chi)$ e todas as suas derivadas também são limitadas em $I$, já que $I$ é limitado, obtemos uma constante $\tilde{\tilde{C}}>0$ que independe de $\phi \in \mathcal{C}_{c}^{\infty}(K)$ e satisfaz

$$
\left|\left\langle u,\left(x-x_{0}\right)^{-1}(1-\chi) \phi\right\rangle\right| \leq \tilde{\widetilde{C}} \sum_{j=0}^{m} \sup _{x \in I}\left|\partial^{j} \phi(x)\right|
$$

para toda $\phi \in \mathcal{C}_{c}^{\infty}(I) \operatorname{com} S(\phi) \subset K$.

Sendo assim, existem $L>0$ e $m \in \mathbb{Z}_{+}$tais que

$$
|\langle v, \phi\rangle| \leq\left|\left\langle u, \chi \psi_{\phi}\left(x-x_{0}\right)\right\rangle\right|+\left|\left\langle u,\left(x-x_{0}\right)^{-1}(1-\chi) \phi\right\rangle\right| \leq L \sum_{j=0}^{m+1} \sup _{x \in I}\left|\partial^{j} \phi(x)\right|
$$

para toda $\phi \in \mathcal{C}_{c}^{\infty}(I)$ com $S(\phi) \subset K$, concluindo que $v \in \mathcal{D}^{\prime}(I)$. Mais ainda, ficou demonstrado que se $u \in \mathcal{D}^{\prime}(I)$ for de ordem finita menor ou igual a $m$, então $v$ será de ordem finita menor ou igual a $m+1$.

Por fim, seja $f \in \mathcal{C}^{\infty}(I)$ uma função que se anule apenas em $x_{0} \in I$, o qual é um zero de ordem finita igual a $n \geq 1$. Utilizando a fómula de Taylor com resto integral podemos escrever $f(x)=\left(x-x_{0}\right)^{n} g(x)$, para todo $x \in I$, com $g \in \mathcal{C}^{\infty}(I)$ e $g^{-1}(0)=\emptyset$. Como a função $g$ não se anula em $I$, existe $v_{0}=(1 / g) u \in \mathcal{D}^{\prime}(I)$ satisfazendo $g v_{0}=u$. Pelo caso anterior, existe $v_{1} \in \mathcal{D}^{\prime}(I)$ tal que $\left(x-x_{0}\right) v_{1}=v_{0}$ e assim $\left(x-x_{0}\right) g v_{1}=u$. Utilizando o caso anterior indutivamente, obtemos uma distribuição $v_{n} \in \mathcal{D}^{\prime}(I)$ satisfazendo $f v_{n}=$ $\left(x-x_{0}\right)^{n} g v_{n}=u$. Se $u \in \mathcal{D}^{\prime}(I)$ for de ordem finita menor ou igual a $m$, então a ordem de $v_{0}$ será menor ou igual a $m$ e $v_{i}$ poderá ser escolhida de ordem menor ou igual a $m+i$, para cada $i=1, \ldots, n$. 
(ii) Fixe $x_{0} \in \mathbb{R} \backslash f^{-1}(0)$ e denote $\left(x_{0}, x_{0}+2 \pi\right) \cap f^{-1}(0)$ por $\left\{x_{1}<\cdots<x_{n}\right\}$. Defina $x_{-1} \doteq x_{n}-2 \pi, x_{n+1} \doteq x_{0}+2 \pi$ e $I_{j} \doteq\left(x_{j-1}, x_{j+1}\right)$, para $j=0, \ldots, n$.

Para cada $j \in\{1, \ldots, n\}$, a função $f$ está em $\mathcal{C}^{\infty}\left(I_{j}\right)$ e se anula apenas em $x_{j} \in I_{j}$, o qual é um zero de ordem finita, digamos $n_{j} \geq 1$. Segue do item (i) que existe $v_{j} \in \mathcal{D}^{\prime}\left(I_{j}\right)$ tal que $f v_{j}=u$ em $\mathcal{D}^{\prime}\left(I_{j}\right)$. Definindo $v_{0} \doteq f^{-1} u \in \mathcal{D}^{\prime}\left(I_{0}\right)$, temos $f v_{0}=u$ em $\mathcal{D}^{\prime}\left(I_{0}\right)$, $v_{j}=v_{j+1}=f^{-1} u \mathrm{em} \mathcal{D}^{\prime}\left(I_{j} \cap I_{j+1}\right), j=0, \ldots, n-1$, e $v_{0}(x-2 \pi)=v_{n}=f^{-1} u \mathrm{em}$ $\mathcal{D}^{\prime}\left(\left(I_{0}+2 \pi\right) \cap I_{n}\right)$.

Observe também que se $u$ for de ordem finita menor ou igual a $m$, então $v_{0}$ será de ordem finita menor ou igual a $m$ e por (i) podemos tomar $v_{j}$ de ordem finita menor ou igual a $m+n_{j}$, para cada $j=1, \ldots, n$.

Coloque $I_{j}^{k} \doteq I_{j}+2 k \pi$, para todo $k \in \mathbb{Z}$ e $j=0 \ldots, n$. Então $\mathbb{R}=\bigcup_{k \in \mathbb{Z}} \bigcup_{j=0}^{n} I_{j}^{k} \mathrm{e}$ $f v_{j}(x-2 k \pi)=u$ em $\mathcal{D}^{\prime}\left(I_{j}^{k}\right)$, pois $f \in \mathcal{P}_{2 \pi}(\mathbb{R})$ e $u \in \mathcal{D}_{2 \pi}^{\prime}(\mathbb{R})$.

Definindo $v_{j}^{k} \doteq v_{j}(x-2 k \pi) \in \mathcal{D}^{\prime}\left(I_{j}^{k}\right)$, para todo $k \in \mathbb{Z}$ e $j=0, \ldots, n$; segue do Teorema (2.22) que existe $v \in \mathcal{D}^{\prime}(\mathbb{R})$ satisfazendo $v=v_{j}^{k}$ em $\mathcal{D}^{\prime}\left(I_{j}^{k}\right)$ e se $u$ for de ordem finita menor ou igual a $m$, segue da Observação (2.23) que $v$ possuirá ordem finita menor ou igual a $m+\max \left\{n_{j} ; j=1, \ldots, n\right\} \leq m+M$.

Do Lema (2.17) resulta que $f v=u$ em $\mathcal{D}^{\prime}(\mathbb{R})$. Falta apenas verificar que $v \in \mathcal{D}_{2 \pi}^{\prime}(\mathbb{R})$ e, para tanto, basta provarmos que $v=v(x-2 \pi)$. Para cada $x \in \mathbb{R}$ existe $j \in\{0, \ldots, n\}$ e $k \in \mathbb{Z}$ tais que $x \in I_{j}^{k}$; assim, para cada $\phi \in \mathcal{C}_{c}^{\infty}\left(I_{j}^{k}\right)$, temos

$$
\begin{gathered}
\langle v, \phi\rangle=\left\langle v_{j}^{k}, \phi\right\rangle=\left\langle v_{j}(x-2 k \pi), \phi\right\rangle=\left\langle v_{j}, \phi(x+2 k \pi)\right\rangle=\left\langle v_{j}, \phi(x+2 \pi+2(k-1) \pi)\right\rangle= \\
\left\langle v_{j}(x-2(k-1) \pi), \phi(x+2 \pi)\right\rangle=\left\langle v_{j}^{k-1}, \phi(x+2 \pi)\right\rangle=\langle v, \phi(x+2 \pi)\rangle=\langle v(x-2 \pi), \phi\rangle .
\end{gathered}
$$

Novamente pelo Lema (2.17) resulta que $v=v(x-2 \pi)$, ou seja, $v \in \mathcal{D}_{2 \pi}^{\prime}(\mathbb{R})$.

2.29 Observação. Se no item $(i)$ do Teorema (2.28) tivermos $x_{0} \in S(u)$, então teremos $S(v)=S(u)$, para toda $v \in \mathcal{D}^{\prime}(I)$ que satisfaz $f v=u$, pois $S(u)=S(f v) \subset S(f) \cap S(v)$ e $S(v) \backslash\left\{x_{0}\right\}=S(u) \backslash\left\{x_{0}\right\}$. Se no item $(i i)$ tivermos $f^{-1}(0) \subset S(u)$, então teremos $S(v) \subset S(u)$, para toda $v \in \mathcal{D}_{2 \pi}^{\prime}(\mathbb{R})$ que satisfaz $f v=u$, logo $S(v)=S(u)$.

Se $v \in \mathcal{D}^{\prime}(I)$ for uma solução do problema de divisão considerado no item $(i)$ do Teorema (2.28), então segue do Teorema (2.27) que todas as outras soluções serão da forma $v+\sum_{0 \leq j \leq n-1} c_{j} \partial^{j} \delta\left(x-x_{0}\right)$.

O próximo teorema exibe casos em que não é possível efetuar a divisão. 
2.30 Teorema. Seja $\Omega \subset \mathbb{R}$ um aberto.

(i) Se $f \in \mathcal{C}^{\infty}(\Omega)$ for flat em $x_{0} \in \Omega$, não existirá $u \in \mathcal{D}^{\prime}(\Omega)$ tal que $f u=1$.

(ii) Se $f \in \mathcal{P}_{T}(\mathbb{R})$ for flat em $x_{0} \in \mathbb{R}$, não existirá $u \in \mathcal{P}_{T}^{\prime}(\mathbb{R})$ tal que $f u=1$.

(iii) Se $f \in \mathcal{C}^{\infty}(\Omega)$ for flat em $x_{0}$, não existirá $u \in \mathcal{D}^{\prime}(\Omega)$ tal que

$$
f u=\sum_{j=0}^{r} c_{j} \partial^{j} \delta\left(x-x_{0}\right)
$$

onde $c_{j} \in \mathbb{C}$, para todo $0 \leq j \leq r \in \mathbb{Z}_{+}$e $c_{r} \neq 0$.

(iv) Se $f \in \mathcal{P}_{T}(\mathbb{R})$ for flat em $x_{0} \in \mathbb{R}$, não existirá $u \in \mathcal{P}_{T}^{\prime}(\mathbb{R})$ tal que

$$
f u=\sum_{j=0}^{r} c_{j} \partial^{j} \delta\left(x-x_{0}\right)
$$

onde $c_{j} \in \mathbb{C}$, para todo $0 \leq j \leq r \in \mathbb{Z}_{+}$e $c_{r} \neq 0$.

Prova: (i) Demonstraremos inicialmente o caso em que $x_{0}=0$.

Suponha que exista $u \in \mathcal{D}^{\prime}(\Omega)$ satisfazendo $f u=1$; então $\langle u, f \phi\rangle=\langle 1, \phi\rangle=\int_{\Omega} \phi$, para toda $\phi \in \mathcal{C}_{c}^{\infty}(\Omega)$. Fixe $0<\epsilon<1 / 4$ tal que $K=[0,4 \epsilon] \subset \Omega$. Pela continuidade de $u$, existe uma constante $C>0$ e um inteiro não negativo $m$ tais que

$$
\left|\int_{\Omega} \phi\right|=|\langle u, f \phi\rangle| \leq C \sum_{j=0}^{m} \sup _{x \in \Omega}\left|\partial^{j}(f \phi)(x)\right|,
$$

para toda $\phi \in \mathcal{C}_{c}^{\infty}(K)$

Tome $0 \leq \phi_{0} \in \mathcal{C}_{c}^{\infty}(\mathbb{R})$ com $S\left(\phi_{0}\right) \subset[\epsilon, 4 \epsilon]$ e $\phi_{0} \equiv 1$ em uma vizinhança de $[2 \epsilon, 3 \epsilon]$. Para cada $n \in \mathbb{N}$, defina $\phi_{n}(x)=n \phi_{0}(n x)$, para todo $x \in \mathbb{R}$. Observe que cada $\phi_{n}$ satisfaz $S\left(\phi_{n}\right) \subset[\epsilon / n, 4 \epsilon / n]$ e, portanto, $\phi_{n} \in \mathcal{C}_{c}^{\infty}(\Omega)$.

Como

$$
\left|\int_{\Omega} \phi_{n}\right|=\int_{\Omega} n \phi_{0}(n x) d x \geq n \int_{2 \epsilon / n}^{3 \epsilon / n} 1 d x=\epsilon,
$$

segue de (.3) que

$$
\epsilon \leq C \sum_{j=0}^{m} \sup _{x \in[\epsilon / n, 4 \epsilon / n]}\left|\partial^{j}\left(f \phi_{n}\right)(x)\right|
$$

para todo $n \in \mathbb{N}$.

Utilizando que $f$ se anula de ordem $m+2$ em $x=0$, já que $f$ é flat em $x=0$, segue da fórmula de Taylor com resto integral que $f(x)=x^{m+2} g(x)$, para todo $x \in[0,4 \epsilon]$; 
$\operatorname{com} g \in \mathcal{C}^{\infty}([0,4 \epsilon])$. Assim,

$$
\begin{gathered}
C \sum_{j=0}^{m} \sup _{x \in[\epsilon / n, 4 \epsilon / n]}\left|\partial^{j}\left(f \phi_{n}\right)(x)\right| \leq C \sum_{j=0}^{m} \sum_{l=0}^{j}\left(\begin{array}{l}
j \\
l
\end{array}\right) \sup _{x \in[\epsilon / n, 4 \epsilon / n]}\left|\partial^{j-l}\left(x^{m+2}\right) \partial^{l}\left(g(x) n \phi_{0}(n x)\right)\right| \leq \\
C(m+2) ! \sum_{j=0}^{m} n j ! \sum_{l=0}^{j} \sup _{x \in[\epsilon / n, 4 \epsilon / n]}\left[|x|^{m+2-j+l} \sum_{k=0}^{l}\left(\begin{array}{l}
l \\
k
\end{array}\right)\left|\left(\partial^{k} g\right)(x) n^{l-k}\left(\partial^{l-k} \phi_{0}\right)(n x)\right|\right] \leq \\
C(m+2) ! \sum_{j=0}^{m} n(j !)^{2} \sum_{l=0}^{j} \sum_{k=0}^{l} n^{l} \sup _{x \in[\epsilon / n, 4 \epsilon / n]}|x|^{m+2-j+l} \cdot\left|\left(\partial^{k} g\right)(x)\right| \cdot\left|\left(\partial^{l-k} \phi_{0}\right)(n x)\right| \leq \\
C(m+2) ! \sum_{j=0}^{m} n(j !)^{2} \sum_{l=0}^{j} \sum_{k=0}^{l}(n)^{-m-2+j}\left(\sup _{x \in[0,4 \epsilon]}\left|\left(\partial^{k} g\right)(x)\right|\right) \cdot\left\|\partial^{l-k} \phi_{0}\right\|_{\infty} \leq \\
C(m+2) ! \sum_{j=0}^{m}(j !)^{2}(n)^{-m-2+j+1} \sum_{l=0}^{j} \sum_{k=0}^{l}\left(\sup _{x \in[0,4 \epsilon]}\left|\left(\partial^{k} g\right)(x)\right|\right) \cdot\left\|\partial^{l-k} \phi_{0}\right\|_{\infty} \leq \\
n^{-1} C(m+2) ! \sum_{j=0}^{m}(j !)^{2} \sum_{l=0}^{j} \sum_{k=0}^{l}\left(\sup _{x \in[0,4 \epsilon]}\left|\left(\partial^{k} g\right)(x)\right|\right) \cdot\left\|\partial^{l-k} \phi_{0}\right\|_{\infty}
\end{gathered}
$$

e, portanto, a sequência $C \sum_{j=0}^{m} \sup _{x \in \Omega}\left|\partial^{j}\left(f \phi_{n}\right)(x)\right|$ converge para 0 , o que é uma contradição $\operatorname{com}(.4)$

Para concluir o caso geral, suponha que exista $u \in \mathcal{D}^{\prime}(\Omega)$ satisfazendo $f u=1$. Então $u\left(x+x_{0}\right) \in \mathcal{D}^{\prime}\left(\Omega-x_{0}\right)$ satisfaz $f\left(x+x_{0}\right) u\left(x+x_{0}\right)=1$, onde $f\left(x+x_{0}\right) \in \mathcal{C}^{\infty}\left(\Omega-x_{0}\right)$ é flat em $0 \in \Omega-x_{0}$. Do caso anterior segue que tal $u$ não pode existir.

(ii) Suponha que exista $u \in \mathcal{P}_{T}^{\prime}(\mathbb{R})$ satisfazendo $f u=1$ em $\mathcal{P}_{T}^{\prime}(\mathbb{R})$. Aplicando o isomorfismo ${ }^{t} H$ na identidade anterior obtemos $f{ }^{t} H(u)={ }^{t} H(f u)=1$ em $\mathcal{D}_{T}^{\prime}(\mathbb{R})$, o que é uma contradição com o item $(i)$.

(iii) Utilizando composição de distribuições com translações, como foi feito no fim da demonstração do item (i), podemos supor que $x_{0}=0 \in \Omega$. Suponha que exista $u \in \mathcal{D}^{\prime}(\Omega)$ satisfazendo $f u=\sum_{j=0}^{r} c_{j} \partial^{j} \delta$; então

$$
\langle u, f \phi\rangle=\left\langle\sum_{j=0}^{r} c_{j} \partial^{j} \delta, \phi\right\rangle=\sum_{j=0}^{r} c_{j}(-1)^{j}\left(\partial^{j} \phi\right)(0)
$$


para toda $\phi \in \mathcal{C}_{c}^{\infty}(\Omega)$. Fixe $0<\epsilon<1$ tal que $[-\epsilon, \epsilon] \subset \Omega$. Segue da continuidade de $u$ que existe uma constante $C>0$ e um inteiro não negativo $m$ tais que

$$
\left|\sum_{j=0}^{r} c_{j}(-1)^{j}\left(\partial^{j} \phi\right)(0)\right|=|\langle u, f \phi\rangle| \leq C \sum_{j=0}^{m} \sup _{x \in \Omega}\left|\partial^{j}(f \phi)(x)\right|
$$

para toda $\phi \in \mathcal{C}_{c}^{\infty}(\Omega) \operatorname{com} S(\phi) \subset[-\epsilon, \epsilon]$.

Seja $\phi_{0} \in \mathcal{C}_{c}^{\infty}(\mathbb{R})$, dada por $\phi_{0}(x)=x^{r} \psi_{0}(x)$, onde $\psi_{0} \in \mathcal{C}_{c}^{\infty}((-\epsilon, \epsilon))$ é uma função de corte constante igual a 1 em uma vizinhança de $[-\epsilon / 2, \epsilon / 2]$. Então $\phi_{0} \in \mathcal{C}_{c}^{\infty}((-\epsilon, \epsilon))$, satisfaz $\left(\partial^{j} \phi_{0}\right)(0)=0$, se $j \neq r$ e $\left(\partial^{r} \phi_{0}\right)(0)=r$ !. Defina $\phi_{n}(x)=\phi_{0}(n x)$, para todo $x \in \mathbb{R}$ e $n \in \mathbb{N}$. Então $\phi_{n} \in \mathcal{C}_{c}^{\infty}(\Omega)$ satisfaz $S\left(\phi_{n}\right) \subset[-\epsilon / n, \epsilon / n]$.

Podemos então aplicar a desigualdade (.5) a cada $\phi_{n}$, resultando que

$$
\left|c_{r}\right| r ! n^{r} \leq C \sum_{j=0}^{m} \sup _{x \in[-\epsilon / n, \epsilon / n]}\left|\partial^{j}\left(f \phi_{n}\right)(x)\right|
$$

para todo $n \in \mathbb{N}$.

Utilizando que $f$ se anula de ordem $m+1$ em $x=0$, escrevemos $f(x)=x^{m+1} g(x)$, para todo $x \in(-\epsilon, \epsilon)$, onde $g \in \mathcal{C}^{\infty}((-\epsilon, \epsilon))$. Assim,

$$
C \sum_{j=0}^{m} \sup _{x \in[-\epsilon / n, \epsilon / n]}\left|\partial^{j}\left(f \phi_{n}\right)(x)\right|=C \sum_{j=0}^{m} \sup _{x \in[-\epsilon / n, \epsilon / n]}\left|\partial^{j}\left(x^{m+1} g(x) \phi_{0}(n x)\right)\right|
$$

e efetuando cálculos análogos aos da demonstração do item (i) obtemos

$$
\begin{gathered}
C \sum_{j=0}^{m} \sup _{x \in[-\epsilon / n, \epsilon / n]}\left|\partial^{j}\left(f \phi_{n}\right)(x)\right| \leq \\
C(m+1) ! \sum_{j=0}^{m}(j !)^{2} n^{-m-1+j} \sum_{l \leq j} \sum_{k \leq l}\left(\sup _{x \in[-\epsilon / 2, \epsilon / 2]}\left|\left(\partial^{k} g\right)(x)\right|\right) \cdot\left\|\partial^{l-k} \phi_{0}\right\|_{\infty} \leq \\
n^{-1} C(m+1) ! \sum_{j=0}^{m}(j !)^{2} \sum_{l \leq j} \sum_{k \leq l}\left(\sup _{x \in[-\epsilon / 2, \epsilon / 2]}\left|\left(\partial^{k} g\right)(x)\right|\right) \cdot\left\|\partial^{l-k} \phi_{0}\right\|_{\infty} .
\end{gathered}
$$

Resulta então que a sequência $C \sum_{j=0}^{m} \sup _{x \in[-\epsilon / n, \epsilon / n]}\left|\partial^{j}\left(f \phi_{n}\right)(x)\right|$ converge para 0 , o que é uma contradição com (.6), pois $c_{r} \neq 0$. 
(iv) Suponha que exista $u \in \mathcal{P}_{T}^{\prime}(\mathbb{R})$ satisfazendo $f u=\sum_{j=0}^{r} c_{j} \partial^{j} \delta\left(x-x_{0}\right)$. Aplicando o isomorfismo ${ }^{t} H$ na identidade anterior obtemos $f{ }^{t} H(u)=\sum_{j=0}^{r} c_{j} \partial^{j}\left({ }^{t} H \delta\right)\left(x-x_{0}\right)$ em $\mathcal{D}_{T}^{\prime}(\mathbb{R})$. Restringindo a identidade anterior ao intervalo $\left(x_{0}-T / 2, x_{0}+T / 2\right)$, obtemos $f^{t} H(u)=\sum_{j=0}^{r} c_{j} \partial^{j} \delta\left(x-x_{0}\right)$ em $\mathcal{D}^{\prime}\left(x_{0}-T / 2, x_{0}+T / 2\right)$, o que é uma contradição com o item $($ iii).

As mesmas técnicas que aparecem na demonstração do teorema anterior são utilizadas para demonstrar as seguintes proposições.

2.31 Proposição. Sejam $(c, d)$ um intervalo aberto de $\mathbb{R}, u \in \mathcal{D}^{\prime}((c, d))$ e $f \in \mathcal{C}^{\infty}(\mathbb{R})$ uma função flat em c tal que $f u=1 \mathrm{em} \mathcal{D}^{\prime}((c, d))$. Então, para qualquer $\epsilon>0$, u não admite extens $\tilde{a} o \tilde{u} \in \mathcal{D}^{\prime}((c-\epsilon, d))$.

2.32 Proposição. Seja $I \subset \mathbb{R}$ um intervalo aberto contendo $x_{0}$ e seja $f \in \mathcal{C}^{\infty}(I)$ uma função flat em $x_{0}$. Então não existe $u \in \mathcal{D}^{\prime}(I)$ tal que $f u=\delta\left(x-x_{0}\right)+c$, qualquer que $\operatorname{seja} c \in \mathbb{C}$.

\section{Distribuições no toro}

Seja $X$ uma variedade suave $N$-dimensional. Uma carta local em $X$ será denotada por $(U, x)$, onde $x=\left(x_{1}, \ldots, x_{N}\right): U \subset X \rightarrow \mathbb{R}^{N}$.

Denotaremos por $\mathcal{C}^{\infty}(X)$ o espaço das funções $f: X \rightarrow \mathbb{C}$ infinitamente diferenciáveis. Por definição, um campo vetorial complexo e suave em $X$ é uma aplicação $\mathbb{C}$-linear $L: \mathcal{C}^{\infty}(X) \rightarrow \mathcal{C}^{\infty}(X)$ que satisfaz a regra de Leibniz $L(f g)=f L g+g L f$. O conjunto dos campos vetoriais complexos e suaves em $X$ será denotado por $\mathfrak{X}(X)$.

Se $W \subset X$ for um aberto, a restrição de $L \in \mathfrak{X}(X)$ ao aberto $W$ será o campo $L_{W} \in \mathfrak{X}(W)$, dado por $L_{W} f(q)=L \tilde{f}(q)$, onde $\tilde{f} \in \mathcal{C}^{\infty}(X)$ e $\tilde{f} \equiv f$ em um aberto contendo $q$.

Verifica-se que a aplicação $\mathbb{C}$-linear $\partial_{x_{j}}=\frac{\partial}{\partial x_{j}}: \mathcal{C}^{\infty}(U) \rightarrow \mathcal{C}^{\infty}(U)$, dada por $\partial_{x_{j}} f \doteq$ $\left(\partial_{x_{j}}\left(f \circ x^{-1}\right)\right) \circ x$, é um campo vetorial complexo suave em $U$. Mais ainda, verifica-se que

$$
L f(q)=\sum_{j=1}^{N} L x_{j}(q) \partial_{x_{j}} f(q)
$$


qualquer que seja $q \in U$. Tal expressão é chamada de representação do campo $L$ nas coordenadas locais $(U, x)$.

Um campo $L \in \mathfrak{X}(X)$ será dito real quando satisfizer $L \mathcal{C}^{\infty}(X, \mathbb{R}) \subset \mathcal{C}^{\infty}(X, \mathbb{R})$.

Considere em $\mathbb{R}^{N}$ a relação de equivalência $x \sim y$ se, e somente se, $x-y \in 2 \pi \mathbb{Z}^{N}$ e denote o conjunto das classes de equivalência por $\mathbb{R}^{N} / 2 \pi \mathbb{Z}^{N}$.

A aplicação canônica $\mathfrak{p}: \mathbb{R}^{N} \rightarrow \mathbb{R}^{N} / 2 \pi \mathbb{Z}^{N}$, definida por

$$
x \rightarrow \mathfrak{p}(x) \doteq[x]=\left\{y \in \mathbb{R}^{N} ; x-y \in 2 \pi \mathbb{Z}^{N}\right\},
$$

é sobrejetora. Consideraremos em $\mathbb{R}^{N} / 2 \pi \mathbb{Z}^{N}$ a topologia quociente induzida por $\mathfrak{p}$ e chamaremos tal espaço topológico de toro, o qual denotaremos por $\mathbb{T}^{N}$.

A sobrejeção $\mathfrak{p}$ é na verdade um homeomorfismo local, sendo assim, quando tratarmos de propriedades locais faremos um abuso de notação e denotaremos um ponto $q=\mathfrak{p}\left(x_{1}, \ldots, x_{N}\right)$ simplesmente por $\left(x_{1}, \ldots, x_{N}\right)$. Também omitiremos a notação $\mathfrak{p}$ da imagem de um intervalo $I \subset \mathbb{R}$ de comprimento menor que $2 \pi$, denotando $\mathfrak{p}(I) \subset \mathbb{T}^{1}$ apenas por $I \subset \mathbb{T}^{1}$.

Pode-se verificar que $\mathbb{T}^{N}$ é uma variedade suave de dimensão $N$. Certas restrições da aplicação canônica $\mathfrak{p}$ formam um atlas $N$-dimensional $\mathcal{C}^{\infty}$ para $\mathbb{T}^{N}$.

Com o objetivo de simplificarmos notações, utilizaremos a identificação entre $\mathbb{T}^{N}$ e a variedade produto $\mathbb{T}^{1} \times \cdots \times \mathbb{T}^{1}$ ( $N$ - vezes $)$, dada pelo difeomorfismo $\mathcal{C}^{\infty}$ que associa $\left[\left(x_{1}, \ldots, x_{N}\right)\right]$ ao ponto $\left(\left[x_{1}\right], \ldots,\left[x_{N}\right]\right)$. Assim, denotaremos um ponto de $\mathbb{T}^{N}$ simplesmente por $\left(x_{1}, \ldots, x_{N}\right)$, onde $x_{i} \in \mathbb{T}^{1}$.

Denotaremos por $\mathcal{C}^{\infty}\left(\mathbb{T}^{N}\right)$ o espaço das funções $f: \mathbb{T}^{N} \rightarrow \mathbb{C}$ que são de classe $\mathcal{C}^{\infty}$. Cada função $f$ definida em $\mathbb{T}^{N}$ pode ser identificada com uma única função

$$
\Lambda(f) \doteq f \circ \mathfrak{p}
$$

definida em $\mathbb{R}^{N}$ e $2 \pi$-periódica em cada variável. Como a aplicação quociente $\mathfrak{p}$ é uma submersão sobrejetiva $\mathcal{C}^{\infty}$, segue que $f \in \mathcal{C}^{\infty}\left(\mathbb{T}^{N}\right)$ se, e somente se, $\Lambda(f) \in \mathcal{P}_{2 \pi}\left(\mathbb{R}^{N}\right)$. Obtemos assim um isomorfismo entre tais espaços vetoriais, com isomorfismo inverso dado por $\Lambda^{-1}(g)(\mathfrak{p}(x)) \doteq g(x)$, para toda $g \in \mathcal{P}_{2 \pi}\left(\mathbb{R}^{N}\right)$.

O espaço $L^{p}\left(\mathbb{T}^{N}\right)(1 \leq p \leq \infty)$ é formado pelas funções $f: \mathbb{T}^{N} \rightarrow \mathbb{C}$ tais que a função $f \circ \mathfrak{p}: \mathbb{R}^{N} \rightarrow \mathbb{C}$ é Lebesgue mensurável e $f \circ \mathfrak{p} \in L^{p}\left((0,2 \pi)^{N}\right)$. 
Sejam $f \in \mathcal{C}^{\infty}\left(\mathbb{T}^{N}\right), q \in \mathbb{T}^{N}$ e $v \in T_{q} \mathbb{T}^{N}$ tangente a $\mathbb{T}^{N}$ em $q$. A derivada direcional de $f$ na direção de $v$ em $q$ é o vetor $(f \circ c)^{\prime}(0)$, onde $c$ é uma das curvas que representam $v$. Assim, se $\left(U, \mathfrak{p}^{-1}=\left(x_{1}, \ldots, x_{N}\right)\right)$ for uma carta local que contém $q$, então $\partial_{x_{j}} f(q)$ será a derivada direcional de $f$ na direção do vetor de $T_{q} \mathbb{T}^{N}$ representado pela curva $c(t)=\mathfrak{p}\left(\mathfrak{p}^{-1}(q)+t e_{j}\right)$. Mais ainda, $\partial_{x_{j}} f(q)=\partial_{x_{j}}(f \circ \mathfrak{p})\left(\mathfrak{p}^{-1}(q)\right)=\partial_{x_{j}}(\Lambda(f))\left(\mathfrak{p}^{-1}(q)\right)$, isto é $\partial_{x_{j}} f \in \mathcal{C}^{\infty}\left(\mathbb{T}^{N}\right)$ satisfaz $\Lambda\left(\partial_{x_{j}} f\right)=\partial_{x_{j}}(\Lambda(f))$, para cada $j=1, \ldots, N$.

Fixaremos em $\mathcal{C}^{\infty}\left(\mathbb{T}^{N}\right)$ a topologia metrizável induzida pela sequência de seminormas

$$
|\phi|_{k} \doteq \sup _{\substack{q \in \mathbb{T}^{N} \\|\alpha| \leq k}}\left|\left(\partial^{\alpha} \phi\right)(q)\right|,
$$

onde $\phi \in \mathcal{C}^{\infty}\left(\mathbb{T}^{N}\right)$ e $k \in \mathbb{Z}_{+}$. Deste modo, $\mathcal{C}^{\infty}\left(\mathbb{T}^{N}\right)$ é um espaço topológico metrizável, localmente convexo e completo, isto é, um espaço de Fréchet. Mais ainda, a transformação linear $\Lambda$ que foi definida anteriormente é uma isometria entre os espaços topológicos metrizáveis $\mathcal{C}^{\infty}\left(\mathbb{T}^{N}\right)$ e $\mathcal{P}_{2 \pi}\left(\mathbb{R}^{N}\right)$.

Utilizando tal identificação, podemos transportar para as funções de $\mathcal{C}^{\infty}\left(\mathbb{T}^{N}\right)$ alguns resultados da seção I deste capítulo. Por exemplo, como o isomorfismo $\Lambda$ satisfaz $\Lambda(g h)=$ $\Lambda(g) \Lambda(h)$, segue das proposições (2.8) e (2.9) os seguintes corolários.

2.33 Corolário. Se $g \in \mathcal{C}^{\infty}\left(\mathbb{T}^{1}\right)$ possuir apenas zeros de ordem finita, $f \in \mathcal{C}^{\infty}\left(\mathbb{T}^{1}\right)$ for uma função tal que $g^{-1}(0) \subset f^{-1}(0)$ e a ordem de anulamento de $f$ em cada $x \in g^{-1}(0)$ for maior que a ordem de anulamento da função $g$, então existirá $h \in \mathcal{C}^{\infty}\left(\mathbb{T}^{1}\right)$ tal que $g h=f, f^{-1}(0)=h^{-1}(0)$ e a ordem de anulamento de $h$ em cada zero $x \in h^{-1}(0)$ será igual à ordem de anulamento de $f$ menos a ordem de anulamento de $g$ em $x$.

2.34 Corolário. Se $g \in \mathcal{C}^{\infty}\left(\mathbb{T}^{1}\right)$ possuir apenas zeros de ordem finita e $f \in \mathcal{C}^{\infty}\left(\mathbb{T}^{2}\right)$ for uma função flat em $g^{-1}(0) \times \mathbb{T}^{1}$, então existirá $h \in \mathcal{C}^{\infty}\left(\mathbb{T}^{2}\right)$ flat em $g^{-1}(0) \times \mathbb{T}^{1}$ e tal que $g(x) h(x, t)=f(x, t)$, para todo $(x, t) \in \mathbb{T}^{2}$.

Utilizando o Teorema (2.7) pode-se demonstrar a seguinte proposição.

2.35 Proposição. Se $V \subset \mathbb{T}^{2}$ for um aberto contendo o conjunto $\left\{x_{0}\right\} \times \mathbb{T}^{1} \subset \mathbb{T}^{2} e$ $\left(v_{j}\right)_{j \in \mathbb{Z}_{+}} \subset \mathcal{C}^{\infty}\left(\mathbb{T}^{1}\right)$, então existirá $v \in \mathcal{C}^{\infty}\left(\mathbb{T}^{2}\right) \operatorname{com} S(v) \subset V e$

$$
\left(\partial_{x}^{j} v\right)\left(x_{0}, \cdot\right) \equiv v_{j}
$$

para todo $j \in \mathbb{Z}_{+}$.

O espaço das distribuições em $\mathbb{T}^{N}$ será o dual de $\mathcal{C}\left(\mathbb{T}^{N}\right)$ e será denotado por $\mathcal{D}^{\prime}\left(\mathbb{T}^{N}\right)$. 
Das proposições $A .2$ e $A .2^{\prime}$ de [7] segue que um funcional linear $u: \mathcal{C}^{\infty}\left(\mathbb{T}^{N}\right) \rightarrow \mathbb{C}$ será uma distribuição se, e somente se, existirem $C>0$ e $k \in \mathbb{N}$ tais que

$$
|\langle u, \phi\rangle| \leq C \sum_{|\alpha| \leq k} \sup _{q \in \mathbb{T}^{N}}\left|\left(\partial^{\alpha} \phi\right)(q)\right|
$$

para toda $\phi \in \mathcal{C}^{\infty}\left(\mathbb{T}^{N}\right)$.

Fixaremos em $\mathcal{D}^{\prime}\left(\mathbb{T}^{N}\right)$ a topologia forte. Dessa forma, os transpostos ${ }^{t} \Lambda$ e ${ }^{t} \Lambda^{-1}$ fornecem um isomorfismo entre $\mathcal{D}^{\prime}\left(\mathbb{T}^{N}\right)$ e o dual forte de $\mathcal{P}_{2 \pi}\left(\mathbb{R}^{N}\right)$. Consequentemente, $\mathcal{D}^{\prime}\left(\mathbb{T}^{N}\right)$ munido da topologia forte é isomorfo a $\mathcal{D}_{2 \pi}^{\prime}\left(\mathbb{R}^{N}\right)$, onde $\mathcal{D}_{2 \pi}^{\prime}\left(\mathbb{R}^{N}\right)$ é um subespaço do dual forte de $\mathcal{C}_{c}^{\infty}\left(\mathbb{R}^{N}\right)$. Analogamente, os espaços vetoriais topológicos $\mathcal{D}^{\prime}\left(\mathbb{T}^{N}\right)$, $\mathcal{P}_{2 \pi}^{\prime}\left(\mathbb{R}^{N}\right)$ e $\mathcal{D}_{2 \pi}^{\prime}\left(\mathbb{R}^{N}\right)$ são todos isomorfos, quando consideramos neles a topologia fraca*.

A Proposição (2.14) e o isomorfismo entre os duais forte $\mathcal{D}^{\prime}\left(\mathbb{T}^{N}\right)$ e $\mathcal{D}_{2 \pi}^{\prime}\left(\mathbb{R}^{N}\right)$ fornecem que uma sequência $\left(u_{n}\right)_{n \in \mathbb{N}} \subset \mathcal{D}^{\prime}\left(\mathbb{R}^{N}\right)$ converge para $u \in \mathcal{D}^{\prime}\left(\mathbb{R}^{N}\right)$ se, e somente se, $\left\langle u_{n}-u, \phi\right\rangle \rightarrow 0$, qualquer que seja $\phi \in \mathcal{C}^{\infty}\left(\mathbb{T}^{N}\right)$.

É importante observar que $\mathcal{C}^{\infty}\left(\mathbb{T}^{N}\right)$ é reflexivo. De fato, como $\mathcal{C}^{\infty}\left(\mathbb{T}^{N}\right)$ é um espaço de Fréchet, da Proposição (8.3) de [17] segue que $\mathcal{C}^{\infty}\left(\mathbb{T}^{N}\right)$ é um espaço de Baire. A Proposição (33.2) de [17] prova que todo espaço de Baire é tonelado (veja a definição de espaço tonelado na referência [17]), $\operatorname{logo} \mathcal{C}^{\infty}\left(\mathbb{T}^{N}\right)$ é um espaço tonelado. Utilizando as mesmas técnicas que aparecem no Exemplo (1.46) da referência [15], as quais envolvem resultados como o Teorema de Ascoli e o processo de diagonalização de Cantor, verificase que $\mathcal{C}^{\infty}\left(\mathbb{T}^{N}\right)$ possui a propriedade de Heine-Borel, ou seja, todo subconjunto fechado e limitado de $\mathcal{C}^{\infty}\left(\mathbb{T}^{N}\right)$ é compacto. Portanto, $\mathcal{C}^{\infty}\left(\mathbb{T}^{N}\right)$ é um espaço de Montel (veja a definição de espaço de Montel na referência [17]). Por fim, o corolário da Proposição (36.9) de [17] afirma que todo espaço de Montel é reflexivo, $\operatorname{logo} \mathcal{C}^{\infty}\left(\mathbb{T}^{N}\right)$ é reflexivo.

Analogamente ao que foi feito na seção anterior para as distribuições em $\mathcal{D}^{\prime}(\Omega)$ e $\mathcal{P}_{T}^{\prime}\left(\mathbb{R}^{N}\right)$, podemos definir em $\mathcal{D}^{\prime}\left(\mathbb{T}^{N}\right)$ a distribuição delta de Dirac, o produto de uma distribuição por uma função de $\mathcal{C}^{\infty}\left(\mathbb{T}^{N}\right)$, a translação de uma distribuição (a translação de uma função $\phi \in \mathcal{C}^{\infty}\left(\mathbb{T}^{N}\right)$ por $x_{0} \in \mathbb{T}^{N}$ é a imagem inversa da translação de $\Lambda \phi$ por um representante de $x_{0}$ ) e podemos também derivar as distribuições em $\mathcal{D}^{\prime}\left(\mathbb{T}^{N}\right)$. Por fim, utilizamos a medida de Lebesgue em $\mathbb{R}^{N}$ para definir a integral de uma função $\phi \in L^{1}\left(\mathbb{T}^{N}\right)$, a qual é dada por $\int_{\mathbb{T}^{N}} \phi=\int_{[0,2 \pi]^{N}}(\Lambda \phi)(x) d x$. Dessa forma, cada função $\phi \in L^{1}\left(\mathbb{T}^{N}\right)$ define uma distribuição em $\mathcal{D}^{\prime}\left(\mathbb{T}^{N}\right)$, dada por $\mathcal{C}^{\infty}\left(\mathbb{T}^{N}\right) \ni \psi \mapsto \int_{\mathbb{T}^{N}} \phi \psi \mathrm{e}$ denotada por $\phi \in \mathcal{D}^{\prime}\left(\mathbb{T}^{N}\right)$. 
Em $\mathcal{D}^{\prime}\left(\mathbb{T}^{N}\right)$ também vale um teorema de localização análogo ao que existe em $\mathcal{D}^{\prime}(\Omega)$, o qual é uma consequência da existência de partições da unidade na variedade compacta $\mathbb{T}^{N}$. Para partições da unidade em variedades citamos a referência [6].

2.36 Teorema. Se v e w são distribuições em $\mathcal{D}^{\prime}\left(\mathbb{T}^{N}\right)$ e cada ponto de $\mathbb{T}^{N}$ possui uma vizinhança aberta $U$ tal que $\langle v, \phi\rangle=\langle w, \phi\rangle$, para toda $\phi \in \mathcal{C}^{\infty}\left(\mathbb{T}^{N}\right)$ tal que $S(\phi) \subset U$, então $v=w$.

Também definimos o suporte de uma distribuição $v$ em $\mathcal{D}^{\prime}\left(\mathbb{T}^{N}\right)$ como sendo o conjunto de pontos que não possuem uma vizinhança $U$ restrita a qual $v$ se anule, ou seja, tal que $\langle v, \phi\rangle=0$, para toda $\phi \in \mathcal{C}^{\infty}\left(\mathbb{T}^{1}\right)$ com $S(\phi) \subset U$. Equivalentemente, o suporte de $v$ é a interseção de todos os fechados fora dos quais $v$ é a distribuição nula. Utilizando partição da unidade em $\mathbb{T}^{N}$, verifica-se que a reunião de abertos onde $v \in \mathcal{D}^{\prime}\left(\mathbb{T}^{N}\right)$ se aula é um aberto onde $v$ se anula, logo existe um maior aberto onde $v$ se anula, que é o complementar do suporte de $v$.

As distribuições de $\mathcal{D}^{\prime}\left(\mathbb{T}^{1}\right)$ suportadas em um ponto também se escrevem como uma combinação linear de derivadas de delta.

2.37 Teorema. Se $w \in \mathcal{D}^{\prime}\left(\mathbb{T}^{1}\right)$ e $S(w) \subset\left\{x_{0}\right\}$, então $w$ é uma combinação linear de derivadas de $\delta\left(x-x_{0}\right)$, isto é,

$$
w=\sum_{j=0}^{r} c_{j} \partial^{j} \delta\left(x-x_{0}\right)
$$

Prova: Para cada $\phi \in \mathcal{C}^{\infty}\left(\mathbb{T}^{1}\right)$, temos

$$
\langle w, \phi\rangle=\left\langle{ }^{t} H\left({ }^{t} \Lambda^{-1} w\right), \xi \Lambda \phi\right\rangle
$$

qualquer que seja $\xi \in \mathcal{U}_{2 \pi}(\mathbb{R})$, conforme Proposição (2.15).

Segue do Exemplo (2.10) que podemos tomar $\xi$ tal que $S(\xi) \subset\left(x_{0}-2 \pi, x_{0}+2 \pi\right)$ e $\xi(x) \equiv 1$ em uma vizinhança de $x_{0} \in \mathbb{R}$.

Como $u={ }^{t} H\left({ }^{t} \Lambda^{-1} w\right) \in \mathcal{D}_{2 \pi}^{\prime}(\mathbb{R}) \subset \mathcal{D}^{\prime}(\mathbb{R})$ e $u=0$ em $\left(x_{0}, x_{0}+2 \pi\right)$, temos $S(u) \subset$ $\left\{x_{0}+2 m \pi ; m \in \mathbb{Z}\right\}$. Utilizando o Teorema (2.24) no intervalo $\left(x_{0}-2 \pi, x_{0}+2 \pi\right)$, obtemos

$$
\begin{gathered}
\left\langle{ }^{t} H\left({ }^{t} \Lambda^{-1} w\right), \xi \Lambda \phi\right\rangle=\sum_{j=0}^{r} c_{j}\left\langle\partial^{j} \delta\left(x-x_{0}\right), \xi \Lambda \phi\right\rangle= \\
\sum_{j=0}^{r}(-1)^{j} c_{j} \xi\left(x_{0}\right)\left(\partial^{j} \phi\right)\left(x_{0}\right)=\left\langle\sum_{j=0}^{r} c_{j} \partial^{j} \delta\left(x-x_{0}\right), \phi\right\rangle,
\end{gathered}
$$


onde $r$ é a ordem da distribuição $u={ }^{t} H\left({ }^{t} \Lambda^{-1} w\right)$. Portanto,

$$
\langle w, \phi\rangle=\left\langle\sum_{j=0}^{r} c_{j} \partial^{j} \delta\left(x-x_{0}\right), \phi\right\rangle,
$$

para toda $\phi \in \mathcal{C}^{\infty}\left(\mathbb{T}^{1}\right)$.

Utilizando a mesma técnica da demonstração da Proposição (2.25), obtemos a seguinte proposição.

2.38 Proposição. Sejam $x_{0} \in \mathbb{T}^{1}, k \in \mathbb{Z} \backslash\{0\}, f \in \mathcal{C}^{\infty}\left(\mathbb{T}^{1}, \mathbb{R}\right)$ tal que $x_{0} \in f^{-1}(0)$, $\mathrm{P}_{k} \doteq d / d x(f \cdot)+(i k \cdot) e \mathrm{~L}_{k} \doteq f(x) d / d x+(i k \cdot) . S e$

$$
w=\sum_{j=0}^{r} c_{j} \partial^{j} \delta\left(x-x_{0}\right) \in \mathcal{D}^{\prime}\left(\mathbb{T}^{1}\right),
$$

então existe

$$
u=\sum_{j=0}^{r} b_{j} \partial^{j} \delta\left(x-x_{0}\right) \in \mathcal{D}^{\prime}\left(\mathbb{T}^{1}\right)
$$

$e$

$$
v=\sum_{j=0}^{r} d_{j} \partial^{j} \delta\left(x-x_{0}\right) \in \mathcal{D}^{\prime}\left(\mathbb{T}^{1}\right)
$$

tal que $\mathrm{P}_{k}(u)=w$ e $\mathrm{L}_{k}(v)=w$.

2.39 Corolário. Se $w \in \mathcal{D}^{\prime}\left(\mathbb{T}^{1}\right)$ e $S(w) \subset\left\{x_{1}, x_{2}\right\}$, então existem u e $v$ em $\mathcal{D}^{\prime}\left(\mathbb{T}^{1}\right)$, ambas suportadas em $\left\{x_{1}, x_{2}\right\}$, tais que $\mathrm{P}_{k} v \doteq(f v)^{\prime}+i k v=w$ e $\mathrm{L}_{k} u \doteq f u^{\prime}+i k u=w$, onde $f \in \mathcal{C}^{\infty}\left(\mathbb{T}^{1}, \mathbb{R}\right)$ satisfaz $\left\{x_{1}, x_{2}\right\} \subset f^{-1}(0)$ e $k \in \mathbb{Z} \backslash\{0\}$.

Prova: Tome $\psi \subset \mathcal{C}^{\infty}\left(\mathbb{T}^{1}\right)$ tal que $S(\psi) \subset \mathbb{T}^{1} \backslash\left\{x_{2}\right\}$ e $\psi \equiv 1$ em uma vizinhança de $x_{1}$. Então as distribuições $\psi w$ e $(1-\psi) w$ satisfazem $S(\psi w) \subset\left\{x_{1}\right\}, S((1-\psi) w) \subset\left\{x_{2}\right\}$ e $w=\psi w+(1-\psi) w$. Segue do Teorema $(2.37)$ que

$$
\psi w=\sum_{j=0}^{r_{1}} c_{j} \partial^{j} \delta\left(x-x_{1}\right)
$$

e

$$
(1-\psi) w=\sum_{j=0}^{r_{2}} d_{j} \partial^{j} \delta\left(x-x_{2}\right) .
$$


Da Proposição (2.38) segue que existem

$$
v_{1}=\sum_{j=0}^{r_{1}} a_{j} \partial^{j} \delta\left(x-x_{1}\right)
$$

e

$$
v_{2}=\sum_{j=0}^{r_{2}} b_{j} \partial^{j} \delta\left(x-x_{2}\right)
$$

tais que $\mathbf{P}_{k} v_{1}=\psi w$ e $\mathbf{P}_{k} v_{2}=(1-\psi) w$. Assim,

$$
v=v_{1}+v_{2} \in \mathcal{D}^{\prime}\left(\mathbb{T}^{1}\right)
$$

satisfaz $S(v) \subset\left\{x_{1}, x_{2}\right\}$ e $\mathrm{P}_{k} v=\mathrm{P}_{k} v_{1}+\mathrm{P}_{k} v_{2}=\psi w+(1-\psi) w=w$.

A demonstração para o operador $L_{k}$ é exatamente a mesma.

2.40 Observação. Note que as distribuições $u, v \in \mathcal{D}^{\prime}\left(\mathbb{T}^{1}\right)$ do Corolário (2.39) satisfazem $\langle u, \phi\rangle=0=\langle v, \phi\rangle$, para toda $\phi \in \mathcal{C}^{\infty}\left(\mathbb{T}^{1}\right)$ flat em $\left\{x_{1}, x_{2}\right\}$.

2.41 Teorema. Sejam $w \in \mathcal{D}^{\prime}\left(\mathbb{T}^{1}\right)$ e $g \in \mathcal{C}^{\infty}\left(\mathbb{T}^{1}\right)$ uma função que se anula apenas em $x_{0} \in \mathbb{T}^{1}$, o qual é um zero de ordem finita $r \geq 1$. Se $g w=0$, então

$$
w=\sum_{j=0}^{r-1} c_{j} \partial^{j} \delta\left(x-x_{0}\right),
$$

onde $c_{j} \in \mathbb{C}$, para $j=0, \ldots, r$.

Prova: Conforme a Proposição (2.15), para cada $\phi \in \mathcal{C}^{\infty}\left(\mathbb{T}^{1}\right)$ temos

$$
\langle w, \phi\rangle=\left\langle{ }^{t} H\left({ }^{t} \Lambda^{-1} w\right), \xi \Lambda \phi\right\rangle
$$

qualquer que seja $\xi \in \mathcal{U}_{2 \pi}(\mathbb{R})$.

Segue do Exemplo (2.10) que podemos tomar $\xi$ tal que $S(\xi) \subset\left(x_{0}-2 \pi, x_{0}+2 \pi\right)$ e $\xi(x) \equiv 1$ em uma vizinhança de $x_{0} \in \mathbb{R}$. Como ${ }^{t} H\left({ }^{t} \Lambda^{-1} w\right) \in \mathcal{D}^{\prime}(\mathbb{R})$ e $(\Lambda g)\left({ }^{t} H\left({ }^{t} \Lambda^{-1} w\right)\right)=$ ${ }^{t} H\left((\Lambda g)\left({ }^{t} \Lambda^{-1} w\right)\right)={ }^{t} H\left({ }^{t} \Lambda^{-1}(g w)\right)={ }^{t} H\left({ }^{t} \Lambda^{-1} 0\right)=0$, utilizando o Teorema (2.27) no intervalo $\left(x_{0}-2 \pi, x_{0}+2 \pi\right)$, obtemos

$$
\left\langle{ }^{t} H\left({ }^{t} \Lambda^{-1} w\right), \xi \Lambda \phi\right\rangle=\sum_{j=0}^{r-1} c_{j}\left\langle\partial^{j} \delta\left(x-x_{0}\right), \xi \Lambda \phi\right\rangle=
$$




$$
\sum_{j=0}^{r-1}(-1)^{j} c_{j} \xi\left(x_{0}\right)\left(\partial^{j} \phi\right)\left(x_{0}\right)=\left\langle\sum_{j=0}^{r-1} c_{j} \partial^{j} \delta\left(x-x_{0}\right), \phi\right\rangle .
$$

Portanto,

$$
\langle w, \phi\rangle=\left\langle\sum_{j=0}^{r-1} c_{j} \partial^{j} \delta\left(x-x_{0}\right), \phi\right\rangle
$$

para toda $\phi \in \mathcal{C}^{\infty}\left(\mathbb{T}^{1}\right)$

Também é possível dividir uma distribuição de $\mathcal{D}^{\prime}\left(\mathbb{T}^{1}\right)$ por uma função que possua apenas zeros de ordem finita e é impossível dividir uma combinação linear não nula de derivadas de $\delta\left(x-x_{0}\right) \in \mathcal{D}^{\prime}\left(\mathbb{T}^{1}\right)$ por uma função que seja flat em $x_{0}$, bem como é impossível dividir uma distribuição constante não nula por uma função que seja flat em algum ponto. De fato, utilizando os isomorfismos entre os espaços $\mathcal{P}_{2 \pi}^{\prime}(\mathbb{R}), \mathcal{D}^{\prime}\left(\mathbb{T}^{1}\right)$ e $\mathcal{D}_{2 \pi}^{\prime}(\mathbb{R})$, podemos obter versões dos teoremas $(2.28)$ e $(2.30)$ em $\mathcal{D}^{\prime}\left(\mathbb{T}^{1}\right)$.

2.42 Teorema. Seja $f \in \mathcal{C}^{\infty}\left(\mathbb{T}^{1}\right)$ uma função que possua apenas zeros de ordem finita. Dada $u \in \mathcal{D}^{\prime}\left(\mathbb{T}^{1}\right)$, existe $v \in \mathcal{D}^{\prime}\left(\mathbb{T}^{1}\right)$ tal que $f v=u$.

2.43 Observação. Qualquer $v \in \mathcal{D}^{\prime}\left(\mathbb{T}^{1}\right)$ que divide $u$ por $f$, isto é, que satisfaz $f v=u$, está suportada em $S(u) \cup f^{-1}(0)$. Em particular, se $f^{-1}(0) \subset S(u)$, então $S(v) \subset S(u)$.

2.44 Teorema. (i) Se $g \in \mathcal{C}^{\infty}\left(\mathbb{T}^{1}\right)$ for flat em algum ponto de $\mathbb{T}^{1}$, não existirá uma distribuição $v \in \mathcal{D}^{\prime}\left(\mathbb{T}^{1}\right)$ tal que gv $=1$.

(ii) Se $g \in \mathcal{C}^{\infty}\left(\mathbb{T}^{1}\right)$ for flat em $x_{0} \in \mathbb{T}^{1}$, não existirá $v \in \mathcal{D}^{\prime}\left(\mathbb{T}^{1}\right)$ tal que

$$
g v=\sum_{j=0}^{r} c_{j} \partial^{j} \delta\left(x-x_{0}\right)
$$

onde $c_{j} \in \mathbb{C}$, para todo $0 \leq j \leq r \in \mathbb{Z}_{+}$e $c_{r} \neq 0$.

As proposições (2.31) e (2.32) também possuem versões em $\mathcal{D}^{\prime}\left(\mathbb{T}^{1}\right)$.

2.45 Proposição. Sejam $(\alpha, \beta) \subset \mathbb{T}^{1}$ um intervalo aberto e $f \in \mathcal{C}^{\infty}\left(\mathbb{T}^{1}\right)$ uma função flat em $\alpha$. Não existe $u \in \mathcal{D}^{\prime}\left(\mathbb{T}^{1}\right)$ tal que $\langle f u, \phi\rangle=\langle 1, \phi\rangle$ para toda $\phi \in \mathcal{C}^{\infty}\left(\mathbb{T}^{1}\right)$ que satisfaz $S(\phi) \subset(\alpha, \beta)$.

Prova: Suponha que exista $u \in \mathcal{D}^{\prime}\left(\mathbb{T}^{1}\right)$ tal que $f u=1 \in \mathcal{D}^{\prime}\left(\mathbb{T}^{1}\right)$. Utilizando o isomorfismo $\Lambda$ obtemos $\Lambda f \in \mathcal{P}_{2 \pi}(\mathbb{R})$, a qual é uma função flat em $\alpha$; onde podemos supor 
$(\alpha, \beta) \subset[0,2 \pi] \subset \mathbb{R}$. Mostremos que ${ }^{t} H\left({ }^{t} \Lambda^{-1} u\right) \in \mathcal{D}_{2 \pi}^{\prime}(\mathbb{R})$ é uma distribuição que satisfaz $(\Lambda f)^{t} H\left({ }^{t} \Lambda^{-1} u\right)=1 \mathrm{em}(\alpha, \beta)$. De fato, para cada $\phi \in \mathcal{C}_{c}^{\infty}(\mathbb{R}) \operatorname{com} S(\phi) \subset(\alpha, \beta)$, temos

$$
\begin{gathered}
\left\langle(\Lambda f)^{t} H\left({ }^{t} \Lambda^{-1} u\right), \phi\right\rangle=\left\langle{ }^{t} \Lambda^{-1} u,(\Lambda f)(H \phi)\right\rangle=\left\langle f u, \Lambda^{-1}(H \phi)\right\rangle=\left\langle 1, \Lambda^{-1}(H \phi)\right\rangle= \\
\int_{0}^{2 \pi} H \phi=\int_{\alpha}^{\beta} \phi=\langle 1, \phi\rangle .
\end{gathered}
$$

Portanto, $(\Lambda f)^{t} H\left({ }^{t} \Lambda^{-1} u\right)=1$ em $(\alpha, \beta)$, uma contradição com a Proposição (2.31).

2.46 Proposição. Seja $f \in \mathcal{C}^{\infty}\left(\mathbb{T}^{1}\right)$ uma função flat em $x_{0}$. Não existe $u \in \mathcal{D}^{\prime}\left(\mathbb{T}^{1}\right)$ tal que $f u=\delta\left(x-x_{0}\right)+c$, qualquer que seja $c \in \mathbb{C}$.

Prova: Suponha que exista $u \in \mathcal{D}^{\prime}\left(\mathbb{T}^{1}\right)$ tal que $f u=\delta\left(x-x_{0}\right)+c$. Então a distribuição $(\Lambda f)^{t} H\left({ }^{t} \Lambda^{-1} u\right) \in \mathcal{D}_{2 \pi}^{\prime}(\mathbb{R})$ é uma distribuição que satisfaz $(\Lambda f)^{t} H\left({ }^{t} \Lambda^{-1} u\right)=$ ${ }^{t} H\left({ }^{t} \Lambda^{-1}\left(\delta\left(x-x_{0}\right)+c\right)\right)={ }^{t} H\left(\delta\left(x-x_{0}\right)+c\right)={ }^{t} H \delta\left(x-x_{0}\right)+c=\delta\left(x-x_{0}\right)+c \mathrm{em}$ $\mathcal{D}^{\prime}\left(\left(x_{0}-\pi, x_{0}+\pi\right)\right)$, o que é uma contradição com a Proposição (2.32).

\section{Série de Fourier e produto tensorial}

Nas seções $I .5$ e $I .6$ da referência [18], o autor detalhou séries de Fourier em $\mathcal{P}_{T}\left(\mathbb{R}^{N}\right)$ e $\mathcal{P}_{T}^{\prime}\left(\mathbb{R}^{N}\right)$. Utilizaremos os isomorfismos $\mathcal{C}^{\infty}\left(\mathbb{T}^{N}\right) \simeq \mathcal{P}_{2 \pi}\left(\mathbb{R}^{N}\right)$ e $\mathcal{D}^{\prime}\left(\mathbb{T}^{N}\right) \simeq \mathcal{P}_{2 \pi}^{\prime}\left(\mathbb{R}^{N}\right)$ para transportar tais resultados para os espaços $\mathcal{C}^{\infty}\left(\mathbb{T}^{N}\right)$ e $\mathcal{D}^{\prime}\left(\mathbb{T}^{N}\right)$. Apesar de utilizarmos o isomorfismo $\Lambda$ entre $\mathcal{C}^{\infty}\left(\mathbb{T}^{N}\right)$ e $\mathcal{P}_{2 \pi}\left(\mathbb{R}^{N}\right)$, muitas vezes faremos um abuso de notação e denotaremos apenas por $\phi \in \mathcal{P}_{2 \pi}\left(\mathbb{R}^{N}\right)$ a função $\Lambda \phi$.

Uma sequência numérica $\left(c_{m}\right)_{m \in \mathbb{Z}^{N}} \subset \mathbb{C}$ será dita rapidamente decrescente se para cada $k \in \mathbb{Z}_{+}$existir $C>0$ tal que

$$
\left|c_{m}\right| \leq C /|m|^{k}
$$

para todo $m \in \mathbb{Z}^{N} \backslash\{0\}$. Diremos que $\left(c_{m}\right)_{m \in \mathbb{Z}^{N}} \subset \mathbb{C}$ será de crescimento lento se existirem constantes $M>0$ e $k \in \mathbb{Z}_{+}$tais que

$$
\left|c_{m}\right| \leq M|m|^{k}
$$

para todo $m \in \mathbb{Z}^{N} \backslash\{0\}$. 
Se $\left(c_{m}\right)_{m \in \mathbb{Z}^{N}} \subset \mathbb{C}$ for uma sequência rapidamente decrescente, então a série $\sum_{m \in \mathbb{Z}^{N}} c_{m} e_{m}$, onde $e_{m} \doteq \Lambda^{-1}\left(e^{i m \cdot x}\right)$, será convergente em $\mathcal{C}^{\infty}\left(\mathbb{T}^{N}\right)$. Por outro lado, para cada função $\phi \in \mathcal{C}^{\infty}\left(\mathbb{T}^{N}\right)$, defina

$$
\hat{\phi}(m) \doteq(2 \pi)^{-N} \int_{\mathbb{T}^{N}} \phi e_{-m}=(2 \pi)^{-N} \int_{[0,2 \pi]^{N}} \phi(x) e^{-i m \cdot x} d x
$$

para todo $m \in \mathbb{Z}^{N}$. A sequência numérica $(\hat{\phi}(m))_{m \in \mathbb{Z}^{N}}$ é rapidamente decrescente e, assim, $\sum_{m \in \mathbb{Z}^{N}} \hat{\phi}(m) e_{m}$ converge em $\mathcal{C}^{\infty}\left(\mathbb{T}^{N}\right)$. Como vale a unicidade dos coeficientes de Fourier, concluímos que $\phi=\sum_{m \in \mathbb{Z}^{N}} \hat{\phi}(m) e_{m}$, com convergência em $\mathcal{C}^{\infty}\left(\mathbb{T}^{N}\right)$. A unicidade dos coeficientes de Fourier garante que se $\psi \in \mathcal{C}^{\infty}\left(\mathbb{T}^{N}\right)$ e $\hat{\psi}(m)=0$, para todo $m \in \mathbb{Z}^{N}$, então $\phi \equiv 0$.

A série $\sum_{m \in \mathbb{Z}^{N}} \hat{\phi}(m) e_{m}$ é chamada de série de Fourier da função $\phi$ e cada coeficiente $\hat{\phi}(m)$ é chamado de coeficiente de Fourier da função $\phi$.

Suponha agora que $\left(c_{m}\right)_{m \in \mathbb{Z}^{N}} \subset \mathbb{C}$ seja uma sequência de crescimento lento, então a série $\sum_{m \in \mathbb{Z}^{N}} c_{m} e_{m}$ será convergente em $\mathcal{D}^{\prime}\left(\mathbb{T}^{N}\right)$ e se $u=\sum_{m \in \mathbb{Z}^{N}} c_{m} e_{m}$, então

$$
c_{m}=(2 \pi)^{-N}\left\langle u, e_{-m}\right\rangle \doteq \hat{u}(m)
$$

para cada $m \in \mathbb{Z}^{N}$. Por outro lado, se $u \in \mathcal{D}^{\prime}\left(\mathbb{T}^{N}\right)$, então $\hat{u}(m)=(2 \pi)^{-N}\left\langle u, e_{-m}\right\rangle$ forma uma sequência de crescimento lento e $u=\sum_{m \in \mathbb{Z}^{N}} \hat{u}(m) e_{m}$, com convergência em $\mathcal{D}^{\prime}\left(\mathbb{T}^{N}\right)$, uma vez que também vale a unicidade dos coeficientes $\hat{u}(m)$. A série $\sum_{m \in \mathbb{Z}^{N}} \hat{u}(m) e_{m}$ é chamada de série de Fourier da distribuição $u$ e cada coeficiente $\hat{u}(m)$ é chamado de coeficiente de Fourier da distribuição $u$.

Considere dois abertos $\Omega \subset \mathbb{R}^{p}$ e $\Omega^{\prime} \subset \mathbb{R}^{q}$. Nas referências [1], [11] e [7], os autores apresentaram o produto direto (ou produto tensorial) entre distribuições de $\mathcal{D}^{\prime}(\Omega)$ e distribuições de $\mathcal{D}^{\prime}\left(\Omega^{\prime}\right)$. Utilizando o isomorfismo entre $\mathcal{D}_{2 \pi}^{\prime}\left(\mathbb{R}^{N}\right)$ e $\mathcal{D}^{\prime}\left(\mathbb{T}^{N}\right)$, obtemos o produto tensorial em $\mathcal{D}^{\prime}\left(\mathbb{T}^{N}\right)$.

Sejam $p, q \in \mathbb{N}$ e $N=p+q$. Tome $u \in \mathcal{D}^{\prime}\left(\mathbb{T}^{p}\right), v \in \mathcal{D}^{\prime}\left(\mathbb{T}^{q}\right)$ e $\phi \in \mathcal{C}^{\infty}\left(\mathbb{T}^{N}\right)$. Utilizando o isomorfismo $\mathcal{D}_{2 \pi}^{\prime}\left(\mathbb{R}^{N}\right) \simeq \mathcal{D}^{\prime}\left(\mathbb{T}^{N}\right)$ e o Teorema (4.1.1) de [7], verifica-se que a função $\mathbb{T}^{p} \ni x \rightarrow\langle v, \phi(x, \cdot)\rangle$ pertence a $\mathcal{C}^{\infty}\left(\mathbb{T}^{p}\right)$ e, analogamente, a função $\mathbb{T}^{q} \ni y \rightarrow\langle u, \phi(\cdot, y)\rangle$ 
pertence a $\mathcal{C}^{\infty}\left(\mathbb{T}^{q}\right)$. Mais ainda, para cada $\alpha \in \mathbb{Z}_{+}^{p}$ e $\beta \in \mathbb{Z}_{+}^{q}$, temos $\partial^{\alpha}\langle v, \phi(x, \cdot)\rangle=$ $\left\langle v,\left(\partial_{x}^{\alpha} \phi\right)(x, \cdot)\right\rangle$ e $\partial^{\beta}\langle u, \phi(\cdot, y)\rangle=\left\langle u,\left(\partial_{y}^{\beta} \phi\right)(\cdot, y)\right\rangle$.

Defina $\langle u \otimes v, \phi\rangle=\langle u,\langle v, \phi(x, \cdot)\rangle\rangle$, para toda $\phi \in \mathcal{C}^{\infty}\left(\mathbb{T}^{N}\right)$. Como $u \in \mathcal{D}^{\prime}\left(\mathbb{T}^{p}\right) \mathrm{e}$ $v \in \mathcal{D}^{\prime}\left(\mathbb{T}^{q}\right)$, podemos obter $C>0$ e $k \in \mathbb{N}$ tais que

$$
|\langle u \otimes v, \phi\rangle| \leq C \sum_{\substack{|\alpha| \leq k \\ \alpha \in \mathbb{Z}_{+}^{N}}} \sup _{z \in \mathbb{T}^{N}}\left|\partial^{\alpha} \phi(z)\right|,
$$

para toda $\phi \in \mathcal{C}^{\infty}\left(\mathbb{T}^{N}\right)$. Asssim, $u \otimes v$ é uma distribuição de $\mathcal{D}^{\prime}\left(\mathbb{T}^{N}\right)$, chamada de produto direto entre $u$ e $v$.

Se $\phi \in \mathcal{C}^{\infty}\left(\mathbb{T}^{p}\right)$ e $\psi \in \mathcal{C}^{\infty}\left(\mathbb{T}^{q}\right)$, defina a função $\phi \otimes \psi$ em $\mathcal{C}^{\infty}\left(\mathbb{T}^{p+q}\right)$ por $\phi \otimes \psi(x, y)=$ $\phi(x) \psi(y)$. A distribuição $u \otimes v$ é a única que satisfaz $\langle u \otimes v, \phi \otimes \psi\rangle=\langle u, \phi\rangle \cdot\langle v, \psi\rangle$, uma vez que $\mathcal{C}^{\infty}\left(\mathbb{T}^{p}\right) \otimes \mathcal{C}^{\infty}\left(\mathbb{T}^{q}\right) \subset \mathcal{C}^{\infty}\left(\mathbb{T}^{N}\right)$ é um subespaço denso. Em particular, $u \otimes v=v \otimes u$.

A distribuição $u \otimes v$ também satisfaz $\phi(u \otimes v)=(\phi u) \otimes v$ e $\psi(u \otimes v)=u \otimes(\psi v)$, quaisquer que sejam $\phi \in \mathcal{C}^{\infty}\left(\mathbb{T}^{p}\right)$ e $\psi \in \mathcal{C}^{\infty}\left(\mathbb{T}^{q}\right)$.

Por fim, se $\alpha \in \mathbb{Z}_{+}^{p}$ e $\beta \in \mathbb{Z}_{+}^{q}$, então $\partial^{(\alpha, \beta)}(u \otimes v)=\left(\partial^{\alpha} u\right) \otimes\left(\partial^{\beta} v\right)$.

Continuaremos com $p, q \in \mathbb{N}$ e $N=p+q$. Diremos que uma sequência de funções $\left(\phi_{m}\right)_{m \in \mathbb{Z}^{q}} \subset \mathcal{C}^{\infty}\left(\mathbb{T}^{p}\right)$ será rapidamente decrescente se para cada $\alpha \in \mathbb{Z}_{+}^{p}$ e $n \in \mathbb{Z}_{+}$existir $C>0$ tal que

$$
\left|\partial^{\alpha} \phi_{m}(x)\right| \leq C /|m|^{n}
$$

para todo $x \in \mathbb{T}^{p}$ e $m \in \mathbb{Z}^{q} \backslash\{0\}$.

Dada $\phi \in \mathcal{C}^{\infty}\left(\mathbb{T}^{N}\right)$, temos $\phi(x, \cdot) \in \mathcal{C}^{\infty}\left(\mathbb{T}^{q}\right)$ e, portanto, $\phi(x, \cdot)=\sum_{m \in \mathbb{Z}^{q}} \widehat{\phi(x, \cdot)}(m) e_{m}$. Assim, $\phi(x, y)=\sum_{m \in \mathbb{Z}^{q}} \hat{\phi}_{m}(x) e_{m}(y)$, onde $\hat{\phi}_{m}(x) \doteq \widehat{\phi(x, \cdot)}(m)=(2 \pi)^{-q} \int_{\mathbb{T}^{q}} \phi(x, \cdot) e_{-m}=$ $(2 \pi)^{-q} \int_{[0,2 \pi]^{q}} \phi(x, y) e^{-i m \cdot y} d y$.

Verifica-se que $\hat{\phi}_{m} \in \mathcal{C}^{\infty}\left(\mathbb{T}^{p}\right)$ e que $\partial^{\alpha}\left(\hat{\phi}_{m}\right)(x)=(2 \pi)^{-q} \int_{\mathbb{T}^{q}}\left(\partial^{(\alpha, 0)} \phi\right)(x, \cdot) e_{-m}$, qualquer que seja $\alpha \in \mathbb{Z}_{+}^{p}$. Observe também que se $\phi \in \mathcal{C}^{\infty}\left(\mathbb{T}^{N}\right)$ for tal que $\hat{\phi}_{m} \equiv 0$, para todo $m \in \mathbb{Z}^{q}$, então $\phi$ será a função identicamente nula.

Um teorema afirma que se $\left(\phi_{m}\right)_{m \in \mathbb{Z}^{q}} \subset \mathcal{C}^{\infty}\left(\mathbb{T}^{p}\right)$ for uma sequência rapidamente decrescente de funções, então a série $\sum_{m \in \mathbb{Z}^{q}} \phi_{m} e_{m}$ será convergente em $\mathcal{C}^{\infty}\left(\mathbb{T}^{N}\right)$. Outro teorema afirma que para cada $\phi \in \mathcal{C}^{\infty}\left(\mathbb{T}^{N}\right)$, a sequência $\left(\hat{\phi}_{m}\right)_{m \in \mathbb{Z}^{q}} \subset \mathcal{C}^{\infty}\left(\mathbb{T}^{p}\right)$ é rapidamente decrescente, assim, a série $\sum_{m \in \mathbb{Z}^{q}} \hat{\phi}_{m} e_{m}$ converge em $\mathcal{C}^{\infty}\left(\mathbb{T}^{N}\right)$ e a unicidade dos coeficientes implica que $\sum_{m \in \mathbb{Z}^{q}} \hat{\phi}_{m} e_{m}$ converge para $\phi$ em $\mathcal{C}^{\infty}\left(\mathbb{T}^{N}\right)$. A série $\sum_{m \in \mathbb{Z}^{q}} \hat{\phi}_{m} e_{m}$ é 
chamada série parcial de Fourier na variável y e os coeficientes $\hat{\phi}_{m}$ são ditos coeficientes parciais de Fourier.

Considere agora $u \in \mathcal{D}^{\prime}\left(\mathbb{T}^{N}\right)$ e para cada $\phi \in \mathcal{C}^{\infty}\left(\mathbb{T}^{p}\right)$ defina $u_{\phi}: \mathcal{C}{ }^{\infty}\left(\mathbb{T}^{q}\right) \rightarrow \mathbb{C}$ por $\left\langle u_{\phi}, \psi\right\rangle=\langle u, \phi \otimes \psi\rangle$, para toda $\psi \in \mathcal{C}^{\infty}\left(\mathbb{T}^{q}\right)$.

Utilizando os resultados da seção $I .6$ de [18], verifica-se que $u_{\phi} \in \mathcal{D}^{\prime}\left(\mathbb{T}^{q}\right)$, $\log u_{\phi}=$ $\sum_{m \in \mathbb{Z}^{q}} \widehat{u_{\phi}}(m) e_{m}$ com convergência em $\mathcal{D}^{\prime}\left(\mathbb{T}^{q}\right)$, onde $\widehat{u_{\phi}}(m)=(2 \pi)^{-q}\left\langle u, \phi \otimes e_{-m}\right\rangle$.

Defina $\hat{u}_{m}: \mathcal{C}^{\infty}\left(\mathbb{T}^{p}\right) \rightarrow \mathbb{C}$ por $\left\langle\hat{u}_{m}, \phi\right\rangle \doteq \widehat{u_{\phi}}(m)=(2 \pi)^{-q}\left\langle u, \phi \otimes e_{-m}\right\rangle$, para toda $\phi \in \mathcal{C}^{\infty}\left(\mathbb{T}^{p}\right)$. Então $\hat{u}_{m} \in \mathcal{D}^{\prime}\left(\mathbb{T}^{p}\right)$ e se $u \in \mathcal{D}^{\prime}\left(\mathbb{T}^{N}\right)$ for tal que $\hat{u}_{m}=0$ para todo $m \in \mathbb{Z}^{q}$, então $u$ será a distribuição nula.

Diremos que uma sequência $\left(u_{m}\right)_{m \in \mathbb{Z}^{q}} \subset \mathcal{D}^{\prime}\left(\mathbb{T}^{p}\right)$ será de crescimento lento se existirem $C>0$ e $n \in \mathbb{N}$ tais que

$$
\left|\left\langle u_{m}, \phi\right\rangle\right| \leq C|m|^{n} \sum_{|\alpha| \leq n} \sup _{x \in \mathbb{T}^{p}}\left|\left(\partial^{\alpha} \phi\right)(x)\right|
$$

para toda $\phi \in \mathcal{C}^{\infty}\left(\mathbb{T}^{p}\right)$ e $m \in \mathbb{Z}^{q} \backslash\{0\}$.

Se $u \in \mathcal{D}^{\prime}\left(\mathbb{T}^{N}\right)$, então $\left(\hat{u}_{m}\right)_{m \in \mathbb{Z}^{q}} \subset \mathcal{D}^{\prime}\left(\mathbb{T}^{p}\right)$ será uma sequência de crescimento lento. Por outro lado, se $\left(u_{m}\right)_{m \in \mathbb{Z}^{q}} \subset \mathcal{D}^{\prime}\left(\mathbb{T}^{p}\right)$ for uma sequência de crescimento lento, então a série $\sum_{m \in \mathbb{Z}^{q}} u_{m} \otimes e_{m}$ será convergente em $\mathcal{D}^{\prime}\left(\mathbb{T}^{N}\right)$ e pela unicidade dos coeficientes seguirá que a série $\sum_{m \in \mathbb{Z}^{q}} u_{m} \otimes e_{m}$ convergirá para $u$ em $\mathcal{D}^{\prime}\left(\mathbb{T}^{N}\right)$.

Finalizando essa seção, apresentaremos dois resultados que serão utilizados no capítulo seguinte.

2.47 Proposição. Se $f \in \mathcal{C}^{\infty}\left(\mathbb{T}^{2}\right)$ for flat em $\left\{x_{0}\right\} \times \mathbb{T}^{1} \subset \mathbb{T}^{2}$, então $\hat{f}_{k} \in \mathcal{C} \infty\left(\mathbb{T}^{1}\right)$ será flat em $x_{0} \in \mathbb{T}^{1}$, qualquer que seja $k \in \mathbb{Z}$.

2.48 Proposição. Se $u \in \mathcal{D}^{\prime}\left(\mathbb{T}^{1}\right)$ satisfizer $u^{\prime}=0$, então u será constante, isto é, $u=c$ em $\mathcal{D}^{\prime}\left(\mathbb{T}^{1}\right)$, onde $c \in \mathbb{C}$. 



\begin{tabular}{l|l|}
\cline { 2 - 3 } & Capítulo \\
\cline { 2 - 3 } & 3 \\
\hline
\end{tabular}

\section{Resolubilidade global}

O estudo da resolubilidade global de operadores diferenciais parciais lineares consiste em determinar a sua imagem, sendo que a melhor situação ocorre quando o operador em questão é sobrejetor. Quando o operador não for sobrejetor, podemos questionar se sua imagem será um subespaço fechado, ou ainda, se sua imagem será de codimensão finita.

Os operadores que possuem imagem fechada são ditos globalmente resolúveis e os operadores que possuem imagem de codimensão finita são ditos fortemente resolúveis

Nosso objetivo é estudar a resolubilidade global de uma classe de campos vetoriais reais em $\mathbb{T}_{(x, t)}^{2}$, a saber,

$$
\mathrm{L} \doteq \partial_{t}+a(x) \partial_{x},
$$

onde $a \in \mathcal{C}^{\infty}\left(\mathbb{T}^{1}\right)$ é uma função a valores reais. Para tal estudo, vamos tratar um campo da forma $L$ como um operador que age no espaço de funções $\mathcal{C}^{\infty}\left(\mathbb{T}^{2}\right)$ ou no espaço de distribuições $\mathcal{D}^{\prime}\left(\mathbb{T}^{2}\right)$.

Neste capítulo, estabeleceremos condições necessárias e suficientes para que um operador do tipo $L$ possua imagem fechada, isto é, para que $L$ seja globalmente resolúvel.

A palavra resolúvel está relacionada com o conceito de solução de equações; o que justifica definir como globalmente resolúveis os operadores que possuem imagem fechada 
é a Proposição (1.2). De fato, segue da Proposição (1.2) que L será globalmente resolúvel se, e somente se, para cada $f \in\left(\operatorname{ker}^{t} \mathrm{~L}\right)^{\circ}$ existir $u$ tal que $\mathrm{L} u=f$.

No caso mais simples em que $a^{-1}(0)=\mathbb{T}^{1}$, já é possível verificar que um operador do tipo $L$ não é sobrejetor (ver Apêndice A). Na verdade, nenhum operador do tipo $L$ que age em $\mathcal{C}^{\infty}\left(\mathbb{T}^{2}\right)$ é sobrejetor. Por outro lado, um operador do tipo $L$ agindo em $\mathcal{D}^{\prime}\left(\mathbb{T}^{2}\right)$ é sobrejetor, se e somente se, $\emptyset \neq a^{-1}(0) \neq \mathbb{T}^{1}$ e $a^{-1}(0)$ contém apenas zeros de ordem finita.

No caso em que $a^{-1}(0)=\emptyset$, veremos que um campo vetorial do tipo $L$ se reduz a um campo vetorial com coeficientes constantes do tipo $\tilde{L}=\partial_{x}+\mu \partial_{t}$, onde $\mu \doteq$ $(2 \pi)^{-1} \int_{\mathbb{T}^{1}}(1 / a) \in \mathbb{R}$. Neste caso, veremos que $\mathrm{L}$ será globalmente resolúvel se, e somente se, $\mu$ for um número racional ou um irracional não Liouville.

Dizemos que um número irracional $\gamma$ é um número de Liouville se existe uma sequência $\left(p_{n}, q_{n}\right)_{n \in \mathbb{N}} \subset \mathbb{Z} \times \mathbb{N}$ tal que $q_{n} \rightarrow \infty$ e $\left|\gamma-p_{n} / q_{n}\right|<\left(q_{n}\right)^{-n}$, para todo $n \in \mathbb{N}$.

No caso em que $\emptyset \neq a^{-1}(0) \neq \mathbb{T}^{1}$, a resolubilidade global de $L$ está relacionada com a ordem de anulamento dos zeros da função $a \in \mathcal{C}^{\infty}\left(\mathbb{T}^{1}, \mathbb{R}\right)$.

Utilizando as ferramentas desenvolvidas nos capítulos anteriores, detalharemos a demonstração do seguinte teorema.

3.1 Teorema. Para um operador do tipo $\mathrm{L}=\partial_{t}+a(x) \partial_{x}$, as seguintes propriedades são equivalentes:

(I) $\mathrm{LC}^{\infty}\left(\mathbb{T}^{2}\right)$ é um subespaço fechado de $\mathcal{C}^{\infty}\left(\mathbb{T}^{2}\right)$,

(II) $\mathrm{LD}^{\prime}\left(\mathbb{T}^{2}\right)$ é um subespaço fechado de $\mathcal{D}^{\prime}\left(\mathbb{T}^{2}\right)$,

(III) Uma das seguintes situações ocorre:

(III.1) $a^{-1}(0)=\mathbb{T}^{1}$

(III.2) $a^{-1}(0)=\emptyset$ e $\mu=(2 \pi)^{-1} \int_{\mathbb{T}^{1}}(1 / a)$ é racional ou irracional não Liouville;

(III.3) $\emptyset \neq a^{-1}(0) \neq \mathbb{T}^{1}$ e cada $x \in a^{-1}(0)$ é um zero de ordem finita.

No artigo [3] os autores caracterizaram a resolubilidade forte em $\mathcal{C}^{\infty}\left(\mathbb{T}^{2}\right)$ para campos vetoriais do tipo

$$
\partial_{t}+(a(x)+i b(x)) \partial_{x}
$$


onde $a, b \in \mathcal{C}^{\infty}\left(\mathbb{T}^{1}\right)$ são funções reais. Com os argumentos que aparecem em [3] prova-se que $\mathrm{L}: \mathcal{C}^{\infty}\left(\mathbb{T}^{2}\right) \rightarrow \mathcal{C}^{\infty}\left(\mathbb{T}^{2}\right)$ será fortemente resolúvel se, e somente se, $a^{-1}(0)=\emptyset \mathrm{e}$ $\mu=(2 \pi)^{-1} \int_{\mathbb{T}^{1}}(1 / a)$ for um número irracional não Liouville.

Ao estudarmos a resolubilidade global de um operador do tipo L, aparece, via transposto, uma outra classe de operadores diferenciais parciais lineares de primeira orderm, formada por perturbações dos campos vetoriais do tipo L, a saber,

$$
\mathrm{P} \doteq \partial_{t}+\partial_{x}(a \cdot)
$$

Observe que se considerarmos um operador $P$ agindo em $\mathcal{C}^{\infty}\left(\mathbb{T}^{2}\right)$, então $-{ }^{t} \mathrm{P}=\mathrm{L}$, reciprocamente, se considerarmos $L$ agindo em $\mathcal{C}^{\infty}\left(\mathbb{T}^{2}\right)$, então $-{ }^{t} L=P$. Para a classe de operadores do tipo $\mathbf{P}$ também vale um teorema análogo ao Teorema (3.1).

3.2 Teorema. Para um operador do tipo $\mathrm{P}=\partial_{t}+\partial_{x}(a \cdot)$, as seguintes propriedades são equivalentes:

(I) $\mathrm{PC}^{\infty}\left(\mathbb{T}^{2}\right)$ é um subespaço fechado de $\mathcal{C}^{\infty}\left(\mathbb{T}^{2}\right)$,

(II) $\mathrm{P} \mathcal{D}^{\prime}\left(\mathbb{T}^{2}\right)$ é um subespaço fechado de $\mathcal{D}^{\prime}\left(\mathbb{T}^{2}\right)$,

(III) Uma das seguintes situações ocorre:

(III.1) $a^{-1}(0)=\mathbb{T}^{1}$

(III.2) $a^{-1}(0)=\emptyset$ e $\mu=(2 \pi)^{-1} \int_{\mathbb{T}^{1}}(1 / a)$ é racional ou irracional não Liouville;

(III.3) $\emptyset \neq a^{-1}(0) \neq \mathbb{T}^{1}$ e cada $x \in a^{-1}(0)$ é um zero de ordem finita.

\section{Resolubilidade global no espaço de funções}

Nesta seção, trataremos da resolubilidade global no espaço de funções $\mathcal{C}^{\infty}\left(\mathbb{T}^{2}\right)$. Utilizando a Proposição (1.2), a qual caracteriza o fecho da imagem dos operadores L e P, segue que $L \mathcal{C}^{\infty}\left(\mathbb{T}^{2}\right)$ será um subespaço fechado de $\mathcal{C}^{\infty}\left(\mathbb{T}^{2}\right)$ se, e somente se,

$$
\mathrm{LC}^{\infty}\left(\mathbb{T}^{2}\right) \supset\left(\operatorname{ker}^{t} \mathrm{~L}\right)^{\circ} \doteq \mathcal{E}
$$

e $\mathrm{PC}\left(\mathbb{T}^{2}\right)$ será um subespaço fechado de $\mathcal{C}^{\infty}\left(\mathbb{T}^{2}\right)$ se, e somente se,

$$
\mathrm{PC}^{\infty}\left(\mathbb{T}^{2}\right) \supset\left(\operatorname{ker}^{t} \mathrm{P}\right)^{\circ} \doteq \mathcal{G}
$$


Podemos então reescrever os teoremas (3.1) e (3.2) da seguinte maneira.

3.3 Teorema. Para um operador do tipo $\mathrm{L}=\partial_{t}+a(x) \partial_{x}$, as seguintes propriedades são equivalentes:

(i) $\mathrm{LC} \mathcal{C}^{\infty}\left(\mathbb{T}^{2}\right) \supset\left(\operatorname{ker}^{t} \mathrm{~L}\right)^{\circ}$,

(ii) Uma das seguintes situações ocorre:

(ii.1) $a^{-1}(0)=\mathbb{T}^{1}$

(ii.2) $a^{-1}(0)=\emptyset$ e $\mu=(2 \pi)^{-1} \int_{\mathbb{T}^{1}}(1 / a)$ é racional ou irracional não Liouville;

(ii.3) $\emptyset \neq a^{-1}(0) \neq \mathbb{T}^{1}$ e cada $x \in a^{-1}(0)$ é um zero de ordem finita.

3.4 Teorema. Para um operador do tipo $\mathrm{P}=\partial_{t}+\partial_{x}(a \cdot)$, as seguintes propriedades são equivalentes:

(i) $\mathrm{PC}^{\infty}\left(\mathbb{T}^{2}\right) \supset\left(\operatorname{ker}^{t} \mathrm{P}\right)^{\circ}$,

(ii) Uma das seguintes situações ocorre:

(ii.1) $a^{-1}(0)=\mathbb{T}^{1}$;

(ii.2) $a^{-1}(0)=\emptyset$ e $\mu=(2 \pi)^{-1} \int_{\mathbb{T}^{1}}(1 / a)$ é racional ou irracional não Liouville;

(ii.3) $\emptyset \neq a^{-1}(0) \neq \mathbb{T}^{1}$ e cada $x \in a^{-1}(0)$ é um zero de ordem finita.

Consideraremos inicialmente o caso em que a função $a$ é identicamente nula e o caso em que a função $a$ nunca se anula. Veremos que em tais casos ambos os operadores resumem-se a operadores com coeficientes constantes. De fato, se $a^{-1}(0)=\mathbb{T}^{1}$, então ambos os operadores $\mathrm{L}$ e $\mathrm{P}$ reduzem-se ao operador $Q \doteq \partial_{t}: \mathcal{C}^{\infty}\left(\mathbb{T}^{2}\right) \rightarrow \mathcal{C}^{\infty}\left(\mathbb{T}^{2}\right)$, o qual possui imagem fechada em $\mathcal{C}^{\infty}\left(\mathbb{T}^{2}\right)$, conforme o Teorema (A.1) do Apêndice A.

Agora suponha que $a^{-1}(0)=\emptyset$. Verificar se $\mathrm{L}$ agindo em $\mathcal{C}^{\infty}\left(\mathbb{T}^{2}\right)$ possui imagem fechada equivale a verificar se $L$ agindo em $\mathcal{P}_{2 \pi}\left(\mathbb{R}^{2}\right)$ possui imagem fechada. Podemos multiplicar o campo $\mathrm{L}$ por $a^{-1} \in \mathcal{C}^{\infty}\left(\mathbb{T}^{1}\right)$, obtendo $(1 / a) \mathrm{L}=(1 / a) \partial_{t}+\partial_{x}$ e aplicando a mudança de variáveis $X(x, t)=x, T(x, t)=t+\mu x-\int_{0}^{x} 1 / a$, o campo $(1 / a) \mathrm{L}$ se reduz ao campo $\tilde{\mathrm{L}} \doteq \partial_{X}+\mu \partial_{T}$. De fato, a aplicação $\mathcal{M}: \mathcal{P}_{2 \pi}\left(\mathbb{R}^{2}\right) \rightarrow \mathcal{P}_{2 \pi}\left(\mathbb{R}^{2}\right)$, dada por $\mathcal{M}(\phi)(x, t)=\phi(X(x, t), T(x, t))=\phi\left(x, t+\mu x-\int_{0}^{x} 1 / a\right)$, é um homeomorfismo linear com inverso dado por $\mathcal{M}^{-1}(\psi)(X, T)=\psi(x(X, T), t(X, T))=\psi\left(X, T-\mu X+\int_{0}^{X} 1 / a\right)$ e tal que $\mathcal{M}^{-1} \circ(1 / a) \mathrm{L} \circ \mathcal{M}=\tilde{\mathrm{L}}$. Assim, $\mathrm{L}$ tem imagem fechada se, e somente se, $\tilde{\mathrm{L}}$ tem imagem fechada, o que ocorre se, e somente se, $\mu$ é racional ou irracional não Liouville, conforme o Teorema (A.1) do Apêndice A. 
Analogamente, aplicando a mesma mudança de variáveis para os operadores do tipo $\mathrm{P}$, temos $\mathcal{M}^{-1} \circ \mathrm{P} \circ \mathcal{M}=\tilde{\mathrm{L}} \circ M_{a}$, onde $M_{a}(\phi) \doteq a \phi$ é também um homeomorfismo linear. Assim, P tem imagem fechada se, e somente se, $\tilde{L}$ tem imagem fechada, o que ocorre se, e somente se, $\mu$ é racional ou irracional não Liouville.

Portanto, quando $a^{-1}(0)=\emptyset$, os teoremas (3.3) e (3.4) são uma consequência do Teorema (A.1) do Apêndice A.

As considerações feitas no Apêndice A também implicam que quando $a^{-1}(0)=\mathbb{T}^{1}$ ou $a^{-1}(0)=\emptyset$, nenhum campo do tipo $\mathrm{L}$ ou do tipo $\mathrm{P}$ será sobrejetor. Mais ainda, quando $a^{-1}(0)=\mathbb{T}^{1}$, nenhum campo dos tipos $\mathrm{L}$ ou $\mathrm{P}$ será fortemente resolúvel e quando $a^{-1}(0)=\emptyset$, um campo do tipo $\mathrm{L}$ ou do tipo $\mathrm{P}$ será fortemente resolúvel se, e somente se, $\mu=(2 \pi)^{-1} \int_{\mathbb{T}^{1}}(1 / a) \in \mathbb{R}$ for um número irracional não Liouville.

Consideremos agora o caso em que $\emptyset \neq a^{-1}(0) \neq \mathbb{T}^{1}$. Nosso primeiro objetivo é mostrar que a hipótese de que $a^{-1}(0)$ contém apenas zeros de ordem finita é uma condição suficiente para que a imagem de um operador do tipo $L$ (ou do tipo $P$ ) seja um subespaço fechado de $\mathcal{C}^{\infty}\left(\mathbb{T}^{2}\right)$.

Para cada $f \in \mathcal{E}$ deve existir $u \in \mathcal{C}^{\infty}\left(\mathbb{T}^{2}\right)$ tal que $\mathrm{L} u=f$. Em particular, deve existir $u \in \mathcal{C}^{\infty}\left(\mathbb{T}^{2}\right)$ tal que $\mathrm{L} u-f$ seja flat em $a^{-1}(0) \subset \mathbb{T}^{2}$. O próximo teorema afirma que sempre existe $u \in \mathcal{C}^{\infty}\left(\mathbb{T}^{2}\right)$ tal que $\mathrm{L} u-f$ é flat em $a^{-1}(0) \subset \mathbb{T}^{2}$.

3.5 Teorema. Se $\emptyset \neq a^{-1}(0) \neq \mathbb{T}^{1}$, a possuir apenas zeros de ordem finita e se $f \in \mathcal{E}$, então existirá $u \in \mathcal{C}^{\infty}\left(\mathbb{T}^{2}\right)$ tal que $\mathrm{L} u-f$ será flat em $a^{-1}(0) \subset \mathbb{T}^{2}$.

Prova: Como a função a possui apenas zeros de ordem finita, o conjunto $a^{-1}(0)=$ $\left\{x_{1}, \ldots, x_{N}\right\} \subset \mathbb{T}^{1}$ é finito. Assim sendo, para cada $l=1, \ldots, N$, existe $V_{l}$, vizinhança aberta do conjunto $\left\{x_{l}\right\} \times \mathbb{T}^{1} \subset \mathbb{T}^{2}$, tal que $V_{l} \cap V_{k}=\emptyset$, se $l \neq k$.

Se mostrarmos que existem funções $u_{l} \in \mathcal{C}^{\infty}\left(\mathbb{T}^{2}\right), l=1 \ldots, N$, tais que $S\left(u_{l}\right) \subset V_{l}$ e $\mathrm{L}\left(u_{l}\right)-f$ é flat em $\left\{x_{l}\right\} \times \mathbb{T}^{1} \subset \mathbb{T}^{2}$, então a funcão $u=u_{1}+\cdots+u_{N}$ satisfará a tese do teorema. A existência de tais funções seguirá da Proposição (2.35), o qual é um resultado devido a Borel.

Fixe $x_{l} \in a^{-1}(0)$ um zero de ordem finita $n \geq 1$. Considere $\mathcal{S}$ o $\mathbb{C}$ - espaço vetorial formado pelas sequências $\left(v_{j}\right)_{j \in \mathbb{Z}_{+}} \subset \mathcal{C}^{\infty}\left(\mathbb{T}^{1}\right)$. A aplicação $T: \mathcal{C}^{\infty}\left(\mathbb{T}^{2}\right) \rightarrow \mathcal{S}$, dada por $T v=\left(v_{j}\right)_{j \in \mathbb{Z}_{+}}$, onde $v_{j} \equiv(j !)^{-1}\left(\partial_{x}^{j} v\right)\left(x_{l}, \cdot\right)$, é uma transformação linear que satisfaz $T v=0$ se, e somente se, $v$ é flat em $\left\{x_{l}\right\} \times \mathbb{T}^{1} \subset \mathbb{T}^{2}$.

Denote

$$
T a=\left((j !)^{-1} a^{(j)}\left(x_{l}\right)\right)_{j \in \mathbb{Z}_{+}} \doteq\left(a_{j}\right)_{j \in \mathbb{Z}_{+}} \subset \mathbb{R}
$$


e

$$
T f=\left((j !)^{-1}\left(\partial_{x}^{j} f\right)\left(x_{l}, \cdot\right)\right)_{j \in \mathbb{Z}_{+}} \doteq\left(f_{j}\right)_{j \in \mathbb{Z}_{+}} .
$$

Para $v \in \mathcal{C}^{\infty}\left(\mathbb{T}^{2}\right)$, temos

$$
T(\mathrm{~L} v)=\left(v_{j}^{\prime}+\sum_{m=0}^{j}(j+1-m) a_{m} v_{j+1-m}\right)_{j \in \mathbb{Z}_{+}},
$$

pois

$$
\begin{gathered}
(j !)^{-1}\left(\partial_{x}^{j}\left(\partial_{t} v+a \partial_{x} v\right)\right)\left(x_{l}, \cdot\right)= \\
\partial_{t}\left((j !)^{-1} \partial_{x}^{j} v\right)\left(x_{l}, \cdot\right)+(j !)^{-1} \sum_{m=0}^{j}\left(\begin{array}{c}
j \\
m
\end{array}\right) a^{(m)}\left(x_{l}\right)\left(\partial_{x}^{j+1-m} v\right)\left(x_{l}, \cdot\right)= \\
v_{j}^{\prime}+\sum_{m=0}^{j}(j !)^{-1}\left(\begin{array}{c}
j \\
m
\end{array}\right) m ! a_{m}(j+1-m) ! v_{j+1-m}= \\
v_{j}^{\prime}+\sum_{m=0}^{j}(j+1-m) a_{m} v_{j+1-m},
\end{gathered}
$$

para todo $j \in \mathbb{Z}_{+}$. Assim,

$$
T(\mathbf{L} v-f)=\left(v_{j}^{\prime}+\sum_{m=0}^{j}(j+1-m) a_{m} v_{j+1-m}-f_{j}\right)_{j \in \mathbb{Z}_{+}}
$$

e $\mathrm{L} v-f$ é flat em $\left\{x_{l}\right\} \times \mathbb{T}^{1} \subset \mathbb{T}^{2}$ se, e somente se,

$$
v_{j}^{\prime}+\sum_{m=0}^{j}(j+1-m) a_{m} v_{j+1-m} \equiv f_{j}
$$

para todo $j \in \mathbb{Z}_{+}$.

Uma análise da equação (.1) nos direciona a questionar quais funções de $\mathcal{C}^{\infty}\left(\mathbb{T}^{1}\right)$ possuem primitivas. Utilizando série de Fourier, verifica-se que uma função $g \in \mathcal{C}^{\infty}\left(\mathbb{T}^{1}\right)$ possui primitiva se, e somente se, $\hat{g}(0)=0$. Neste caso, uma primitiva de $g$ é dada por $h=\sum_{k \neq 0}(\hat{g}(k) / i k) e_{k} ; \log g$ possui primitivas de todas as ordens.

Sendo assim, podemos decompor cada função $g \in \mathcal{C}^{\infty}\left(\mathbb{T}^{1}\right)$ como uma soma de uma função constante com uma função que possua primitivas de todas as ordens, a saber, $g=g_{0}+g_{1}$, onde $g_{0} \equiv \hat{g}(0)$ e $g_{1}=\sum_{k \neq 0} \hat{g}(k) e_{k}$. Utilizaremos tal decomposição para as 
funções $v_{j}, f_{j}$ e resolveremos (.1) separadamente para $v_{j, 0}$ e $v_{j, 1}$, isto é, para resolvermos $(.1)$, basta resolvermos as equações

$$
v_{j, 0}^{\prime}+\sum_{m=0}^{j}(j+1-m) a_{m} v_{j+1-m, 0}=\sum_{m=0}^{j}(j+1-m) a_{m} v_{j+1-m, 0}=f_{j, 0}
$$

e

$$
v_{j, 1}^{\prime}+\sum_{m=0}^{j}(j+1-m) a_{m} v_{j+1-m, 1} \equiv f_{j, 1},
$$

para todo $j \in \mathbb{Z}_{+}$.

Como $a_{1}=\cdots=a_{n-1}=0$ e $a_{n} \neq 0$, pois $x_{l}$ é um zero de ordem $n$ de $a$, a equação (.3) é equivalente a

$$
v_{j, 1}^{\prime} \equiv f_{j, 1}, \quad j=0, \ldots, n-1
$$

e

$$
v_{j, 1}^{\prime}+(j+1-n) a_{n} v_{j+1-n, 1}+\cdots+a_{j} v_{1,1} \equiv f_{j, 1}, \quad j \geq n .
$$

Como as funções $f_{j, 1}$ possuem primitivas e as funções de $\mathcal{C}^{\infty}\left(\mathbb{T}^{1}\right)$ que possuem primitivas, possuem primitivas de todas as ordens, podemos determinar indutivamente $v_{0,1}, v_{1,1}, \ldots$ em $\mathcal{C}^{\infty}\left(\mathbb{T}^{1}\right)$, de modo que tais funções satisfaçam as equações acima.

Por fim, a equação (.2) é equivalente a

$$
0=f_{j, 0}, \quad j=0, \ldots, n-1
$$

e

$$
(j+1-n) a_{n} v_{j+1-n, 0}+\cdots+a_{j} v_{1,0}=f_{j, 0}, \quad j \geq n .
$$

Como $a_{n} \neq 0$, podemos determinar indutivamente $v_{1,0}=f_{n, 0}\left(a_{n}\right)^{-1} \mathrm{e}$

$$
v_{k+1,0}=\left[(k+1) a_{n}\right]^{-1}\left(f_{n+k, 0}-\sum_{m=n+1}^{n+k}(n+k+1-m) a_{m} v_{n+k+1-m, 0}\right),
$$

para $k \geq 1$. Note que $v_{0,0}$ pode ser escolhida arbitrariamente.

As condições $0 \equiv f_{j, 0}, j=0, \ldots, n-1$, são chamadas condições de compatibilidade e estão satisfeitas, pois

$$
f_{j, 0}=\hat{f}_{j}(0)=(2 \pi)^{-1} \int_{\mathbb{T}^{1}} f_{j}=(2 \pi j !)^{-1} \int_{\mathbb{T}^{1}}\left(\partial_{x}^{j} f\right)\left(x_{l}, \cdot\right)=(-1)^{j}(2 \pi j !)^{-1}\left\langle\delta^{j}\left(x-x_{l}\right) \otimes 1_{t}, f\right\rangle,
$$


$f \in \mathcal{E}=\left(\operatorname{ker}^{t} \mathrm{~L}\right)^{\circ}$ e $\delta^{j}\left(x-x_{l}\right) \otimes 1_{t} \in \operatorname{ker}^{t} \mathrm{~L}, \operatorname{para} j=0, \ldots, n-1$. De fato, $-{ }^{t} \mathrm{~L}\left(\delta^{j}\left(x-x_{l}\right) \otimes 1_{t}\right)=\delta^{j}\left(x-x_{l}\right) \otimes \partial_{t} 1_{t}+\partial_{x}\left(a \delta^{j}\left(x-x_{l}\right)\right) \otimes 1_{t}=\partial_{x}\left(a \delta^{j}\left(x-x_{l}\right)\right) \otimes 1_{t}=0$,

uma vez que

$$
\begin{gathered}
\left\langle\partial_{x}\left(a \delta^{j}\left(x-x_{l}\right)\right), \phi\right\rangle=-\left\langle\delta^{j}\left(x-x_{l}\right), a \phi^{\prime}\right\rangle=(-1)^{j+1}\left\langle\delta\left(x-x_{l}\right), \partial^{j}\left(a \phi^{\prime}\right)\right\rangle= \\
(-1)^{j+1} \sum_{m=0}^{j}\left(\begin{array}{c}
j \\
m
\end{array}\right) a^{(m)}\left(x_{l}\right) \phi^{(j+1-m)}\left(x_{l}\right)=0,
\end{gathered}
$$

para $j=0, \ldots, n-1$.

Portanto, existem $\left(v_{j, 0}\right)_{j \in \mathbb{Z}_{+}} \subset \mathbb{C}$ e $\left(v_{j, 1}\right)_{j \in \mathbb{Z}_{+}} \subset \mathcal{C}^{\infty}\left(\mathbb{T}^{1}\right)$ que satisfazem (.2) e (.3), respectivamente. Então $\left(v_{j}=v_{j, 0}+v_{j, 1}\right)_{j \in \mathbb{Z}_{+}} \subset \mathcal{C}^{\infty}\left(\mathbb{T}^{1}\right)$ satisfaz (.1). Segue da Proposição (2.35) que existe $u_{l} \in \mathcal{C}^{\infty}\left(\mathbb{T}^{2}\right)$ tal que $S\left(u_{l}\right) \subset V_{l}$ e $\left(\partial_{x}^{j} u_{l}\right)\left(x_{l}, \cdot\right) \equiv(j !) v_{j}$, para todo $j \in \mathbb{Z}_{+}$. Como $\left(v_{j}\right)_{j \in \mathbb{Z}_{+}}$satisfaz (.1), segue que $\mathrm{L}\left(u_{l}\right)-f$ éflat em $\left\{x_{l}\right\} \times \mathbb{T}^{1} \subset \mathbb{T}^{2}$.

Para os operadores do tipo $\mathrm{P}$ também vale um resultado análogo ao Teorema (3.5). Na sua demonstração utilizaremos o seguinte lema.

3.6 Lema. Sejam c um número real não nulo e $f \in \mathcal{C}^{\infty}\left(\mathbb{T}^{1}\right)$. Então existe $u \in \mathcal{C}^{\infty}\left(\mathbb{T}^{1}\right)$ tal que $u^{\prime}+c u=f$.

Prova: Utilizando série de Fourier, para $v \in \mathcal{C}^{\infty}\left(\mathbb{T}^{1}\right)$, temos $v^{\prime}+c v=f$ se, e somente se, $\hat{v}(k)=(c+i k)^{-1} \hat{f}(k)$, para todo $k \in \mathbb{Z}$. Agora, $(\hat{f}(k))_{k \in \mathbb{Z}}$ é uma sequência rapidamente decrescente, $\operatorname{logo}\left((c+i k)^{-1} \hat{f}(k)\right)_{k \in \mathbb{Z}}$ também é uma seqência rapidamente decrescente e, portanto, define uma função $u \in \mathcal{C}^{\infty}\left(\mathbb{T}^{1}\right)$ tal que $\hat{u}(k)=(c+i k)^{-1} \hat{f}(k)$, para todo $k \in \mathbb{Z}, \log 0 u^{\prime}+c u=f$.

3.7 Teorema. Se $\emptyset \neq a^{-1}(0) \neq \mathbb{T}^{1}$, a possuir apenas zeros de ordem finita e se $f \in \mathcal{G}$, então existirá $u \in \mathcal{C}^{\infty}\left(\mathbb{T}^{2}\right)$ tal que $\mathrm{P} u-f$ será flat $e m a^{-1}(0) \subset \mathbb{T}^{2}$.

Prova: Utilizando as mesmas notações e repetindo os mesmos argumentos da demonstração do Teorema (3.5), basta exibir $u_{l} \in \mathcal{C}^{\infty}\left(\mathbb{T}^{2}\right)$ tal que $S\left(u_{l}\right) \subset V_{l}$ e $\mathrm{P}\left(u_{l}\right)-f$ seja flat em $\left\{x_{l}\right\} \times \mathbb{T}^{1} \subset \mathbb{T}^{2}$. Para $v \in \mathcal{C}^{\infty}\left(\mathbb{T}^{2}\right)$, temos

$$
T(\mathrm{P} v-f)=\left(v_{j}^{\prime}+(j+1) \sum_{m=0}^{j+1} a_{m} v_{j-m+1}-f_{j}\right)_{j \in \mathbb{Z}_{+}}
$$


onde $T: \mathcal{C}^{\infty}\left(\mathbb{T}^{2}\right) \rightarrow \mathcal{S}$ é a aplicação definida na demonstração do Teorema (3.5). Assim, $\mathrm{P} v-f$ éflat em $\left\{x_{l}\right\} \times \mathbb{T}^{1} \subset \mathbb{T}^{2}$ se, e somente se,

$$
v_{j}^{\prime}+(j+1) \sum_{m=0}^{j+1} a_{m} v_{j-m+1} \equiv f_{j}
$$

para todo $j \in \mathbb{Z}_{+}$. Decompondo novamente uma função em $\mathcal{C}^{\infty}\left(\mathbb{T}^{1}\right)$ como uma soma de uma constante com uma função que possui primitivas de todas as ordens, segue que para resolvermos (.4), basta resolvermos

$$
(j+1) \sum_{m=0}^{j+1} a_{m} v_{j-m+1,0} \equiv f_{j, 0}
$$

e

$$
v_{j, 1}^{\prime}+(j+1) \sum_{m=0}^{j+1} a_{m} v_{j-m+1,1} \equiv f_{j, 1}
$$

para todo $j \in \mathbb{Z}_{+}$.

Agora, $a_{1}=\cdots=a_{n-1}=0$ e $a_{n} \neq 0$, pois $x_{l}$ é um zero de ordem $n$ de $a$. Assim, se $n=1$, a equação (.6) é equivalente a

$$
v_{j, 1}^{\prime}+(j+1)\left[a_{1} v_{j, 1}+\cdots+a_{j+1} v_{0,1}\right] \equiv f_{j, 1}, \quad j \geq 0
$$

a qual pode ser resolvida, pois do Lema (3.6) segue que podemos determinar indutivamente $v_{0,1}, v_{1,1}, \ldots$ em $\mathcal{C}^{\infty}\left(\mathbb{T}^{1}\right)$ tais que $v_{0,1}^{\prime}+a_{1} v_{0,1} \equiv f_{0,1}$ e $v_{j, 1}^{\prime}+(j+1) a_{1} v_{j, 1} \equiv$ $f_{j, 1}-(j+1)\left[a_{2} v_{j-1,1}+\cdots+a_{j+1} v_{0,1}\right]$, para $j \geq 1$.

Se $n>1$, a equação (.6) é equivalente a

$$
\begin{gathered}
v_{j, 1}^{\prime} \equiv f_{j, 1}, \quad j=0, \ldots, n-2 \\
v_{j, 1}^{\prime}+(j+1)\left[a_{n} v_{j+1-n, 1}+\cdots+a_{j+1} v_{0,1}\right] \equiv f_{j, 1}, \quad j \geq n-1 .
\end{gathered}
$$

Neste caso, como as funções $f_{j, 1}$ possuem primitivas e as funções de $\mathcal{C} \infty\left(\mathbb{T}^{1}\right)$ que possuem primitivas, possuem primitivas de todas as ordens, podemos determinar indutivamente $v_{0,1}, v_{1,1}, \ldots$ em $\mathcal{C}^{\infty}\left(\mathbb{T}^{1}\right)$ que satisfazem as equações acima.

Por fim, se $n=1$, a equação (.5) é equivalente a

$$
a_{1} v_{j, 0}+a_{2} v_{j-1,0}+\cdots+a_{j+1} v_{0,0}=(j+1)^{-1} f_{j, 0}, \quad j \geq 0,
$$


a qual pode ser resolvida tomando $v_{0,0}=a_{1}^{-1} f_{0,0} \mathrm{e}$

$$
v_{j, 0}=a_{1}^{-1}\left[(j+1)^{-1} f_{j, 0}-a_{2} v_{j-1,0}-\cdots-a_{j+1} v_{0,0}\right],
$$

para $j \geq 1$.

Se $n>1$, a equação (.5) é equivalente a

$$
0=f_{j, 0}, \quad j=0, \ldots, n-2
$$

e

$$
a_{n} v_{j+1-n, 0}+a_{n+1} v_{j-n, 0}+\cdots+a_{j+1} v_{0,0}=(j+1)^{-1} f_{j, 0}, \quad j \geq n-1,
$$

a qual pode ser resolvida tomando $v_{j+1-n, 0}=a_{n}^{-1}\left[(j+1)^{-1} f_{j, 0}-a_{n+1} v_{j-n, 0}-\ldots-a_{j+1} v_{0,0}\right]$, para $j \geq n-1$. As condições de compatibilidade $0 \equiv f_{j, 0}$, para $j=0, \ldots, n-2$, estão satisfeitas pois $f \in \mathcal{G}=\left(\operatorname{ker}^{t} \mathrm{P}\right)^{\circ}$ e $\delta^{j}\left(x-x_{l}\right) \otimes 1_{t} \in \operatorname{ker}^{t} \mathrm{P}$, para $j=0, \ldots, n-2$. Tal fato pode ser verificado procedendo de maneira análoga ao que foi feito na demonstração do Teorema (3.5).

Portanto, existem $\left(v_{j, 0}\right)_{j \in \mathbb{Z}_{+}} \subset \mathbb{C}$ e $\left(v_{j, 1}\right)_{j \in \mathbb{Z}_{+}} \subset \mathcal{C}^{\infty}\left(\mathbb{T}^{1}\right)$ que satisfazem (.5) e (.6), respectivamente. Então $\left(v_{j}=v_{j, 0}+v_{j, 1}\right)_{j \in \mathbb{Z}_{+}} \subset \mathcal{C}^{\infty}\left(\mathbb{T}^{1}\right)$ satisfaz (.4). Segue da Proposição (2.35) que existe $u_{l} \in \mathcal{C}^{\infty}\left(\mathbb{T}^{2}\right)$ tal que $S\left(u_{l}\right) \subset V_{l}$ e $\left(\partial_{x}^{j} u_{l}\right)\left(x_{l}, \cdot\right) \equiv(j !) v_{j}$, para todo $j \in \mathbb{Z}_{+}$. Como $\left(v_{j}\right)_{j \in \mathbb{Z}_{+}}$satisfaz (.4), segue que $\mathrm{P}\left(u_{l}\right)-f$ é flat em $\left\{x_{l}\right\} \times \mathbb{T}^{1} \subset \mathbb{T}^{2}$.

3.8 Observação. Se a função $a \in \mathcal{C}^{\infty}\left(\mathbb{T}^{1}\right)$ possui apenas zeros de ordem 1, então não aparecem as condições de compatibilidade e o teorema anterior vale para qualquer $f \in \mathcal{C}^{\infty}\left(\mathbb{T}^{2}\right)$.

Se $\emptyset \neq a^{-1}(0) \neq \mathbb{T}^{1}, a$ possuir apenas zeros de ordem finita e se $f \in \mathcal{E}$, seguirá do Teorema (3.5) que existe $u \in \mathcal{C}^{\infty}\left(\mathbb{T}^{2}\right)$ tal que $\mathrm{L} u-f$ é flat em $a^{-1}(0)$. Dizemos então que o Teorema (3.5) resolve, módulo funções que são flat em $a^{-1}(0) \subset \mathbb{T}^{2}$, o problema de encontrar $u \in \mathcal{C}^{\infty}\left(\mathbb{T}^{2}\right)$ tal que $\mathrm{L} u=f$. Observe que $\mathrm{L} u-f \in \mathcal{E}$, uma vez que $\mathcal{E}$ é um subespaço vetorial e $\mathrm{L} u, f \in \mathcal{E}$. Se existir $v \in \mathcal{C}^{\infty}\left(\mathbb{T}^{2}\right)$ tal que $\mathrm{L} v=\mathrm{L} u-f$, então existirá $u-v \in \mathcal{C}^{\infty}\left(\mathbb{T}^{2}\right)$ tal que $\mathrm{L}(u-v)=f$.

Passaremos então a considerar o caso em que o segundo membro de $L u=f$ é uma função de $\mathcal{E}$, flat em $a^{-1}(0)$.

Para cada $u \in \mathcal{C}^{\infty}\left(\mathbb{T}^{2}\right)$, a série parcial de Fourier na variável $t$ e a continuidade do operador $\mathrm{L}$ fornecem que $\mathrm{L} u=f$ se, e somente se,

$$
a(x) \hat{u}_{k}^{\prime}(x)+i k \hat{u}_{k}(x)=\hat{f}_{k}(x),
$$


para todo $x \in \mathbb{T}^{1}$ e $k \in \mathbb{Z}$. Considere a equação $a(x) \hat{u}_{k}^{\prime}(x)+i k \hat{u}_{k}(x)=\hat{f}_{k}(x)$ em $\mathcal{P}_{2 \pi}(\mathbb{R})$ e suponha que desejamos resolvê-la no intervalo $\left(x_{1}, x_{2}\right)$, onde $x_{1}<x_{2}$ são zeros consecutivos de $a$. Neste caso, fixemos $y_{1}$ e $z_{1}$ em $\left(x_{1}, x_{2}\right)$. Dividindo a equação pela função $a$ e utilizando fator integrante obtemos

$$
\frac{d}{d x}\left(\hat{u}_{k}(x) \exp \left\{i k \int_{y_{1}}^{x} a^{-1}(y) d y\right\}\right)=\hat{f}_{k}(x) a^{-1}(x) \exp \left\{i k \int_{y_{1}}^{x} a^{-1}(y) d y\right\}
$$

para todo $x \in\left(x_{1}, x_{2}\right)$. Equivalentemente, existe $c \in \mathbb{C}$ tal que

$$
\hat{u}_{k}(x) \exp \left\{i k \int_{y_{1}}^{x} a^{-1}(y) d y\right\}=c+\int_{z_{1}}^{x} \hat{f}_{k}(y) a^{-1}(y) \exp \left\{i k \int_{y_{1}}^{y} a^{-1}(z) d z\right\} d y
$$

em $\left(x_{1}, x_{2}\right)$. Então uma solução da equação $a(x) \hat{u}_{k}^{\prime}(x)+i k \hat{u}_{k}(x)=\hat{f}_{k}(x)$ no intervalo $\left(x_{1}, x_{2}\right)$ possui a forma

$$
\hat{u}_{k}(x)=\exp \left\{-i k \int_{y_{1}}^{x} a^{-1}(z) d z\right\}\left(c+\int_{z_{1}}^{x} \hat{f}_{k}(y) a^{-1}(y) \exp \left\{i k \int_{y_{1}}^{y} a^{-1}(z) d z\right\} d y\right) .
$$

Vale notar que qualquer que seja $c \in \mathbb{C}$, a expressão acima satisfaz a equação $a(x) \hat{u}_{k}^{\prime}(x)+$ $i k \hat{u}_{k}(x)=\hat{f}_{k}(x)$ em $\left(x_{1}, x_{2}\right)$.

Desejamos estender suavemente estas soluções a todo o intervalo $\left[x_{1}, x_{2}\right]$, de modo que elas forneçam uma solução definida em $\mathbb{T}^{1}$. A próxima proposição irá auxiliar nessa tarefa.

3.9 Proposição. Seja $b \in \mathcal{C}^{\infty}(\mathbb{R})$ e $x_{1}<x_{2}$ dois zeros de ordem finita de $b$ tais que $\left(x_{1}, x_{2}\right) \cap b^{-1}(0)=\emptyset$. Defina $B(x)=\int_{y}^{x} b^{-1}(z) d z$, para todo $x \in\left(x_{1}, x_{2}\right)$, onde $y \in\left(x_{1}, x_{2}\right)$ é um elemento fixado arbitrariamente. Se g for uma função infinitamente diferenciável em uma vizinhança de $\left[x_{1}, x_{2}\right]$ e flat em $\left\{x_{1}, x_{2}\right\}$, então a função

$$
h(x)=\left\{\begin{aligned}
\exp \{i B(x)\} g(x), & \text { se } x \in\left(x_{1}, x_{2}\right) \\
0, & \text { se } x=x_{1} \text { ou } x=x_{2}
\end{aligned}\right.
$$

está em $\mathcal{C}^{\infty}\left(\left(x_{1}, x_{2}\right)\right)$ e $h^{(n)}\left(x_{1}^{+}\right) \doteq \lim _{x \rightarrow x_{1}^{+}} h^{(n)}(x)=0=\lim _{x \rightarrow x_{2}^{-}} h^{(n)}(x) \doteq h^{(n)}\left(x_{2}^{-}\right)$, para todo $n \in \mathbb{Z}_{+}$.

Prova: Utilizando que $|\exp \{i B(x)\}| \equiv 1$ e que $\lim _{x \rightarrow x_{1}^{+}} g(x)=0=\lim _{x \rightarrow x_{2}^{-}} g(x)$, obtemos $h\left(x_{1}^{+}\right)=0=h\left(x_{2}^{-}\right)$.

Derivando $h$ em $\left(x_{1}, x_{2}\right)$ obtemos $h^{\prime}(x)=\exp \{i B(x)\}\left[g^{\prime}(x)+i g(x) / b(x)\right]$. Como $g$ é flat em $\left\{x_{1}, x_{2}\right\}$ e $b$ se anula de ordem finita, segue da Proposição (2.8) que $h^{\prime}(x)=$ 
$\exp \{i B(x)\} \phi_{1}(x)$, onde $\phi_{1}$ é uma função infinitamente diferenciável em uma vizinhança de $\left[x_{1}, x_{2}\right]$ e flat em $\left\{x_{1}, x_{2}\right\}$. Pelos mesmos argumentos do parágrafo anterior segue que $h^{\prime}\left(x_{1}^{+}\right)=0=h^{\prime}\left(x_{1}^{+}\right)$.

Analogamente, para $n \geq 2$ obtemos $h^{(n)}(x)=\exp \{i B(x)\} \phi_{n}(x)$, para $x \in\left(x_{1}, x_{2}\right)$, onde $\phi_{n}$ é uma função infinitamente diferenciável em uma vizinhança de $\left[x_{1}, x_{2}\right]$ e flat em $\left\{x_{1}, x_{2}\right\}$. Aplicando o mesmo argumento do primeiro parágrafo obtemos $h^{(n)}\left(x_{1}^{+}\right)=$ $0=h^{(n)}\left(x_{2}^{-}\right)$.

3.10 Teorema. Se $\emptyset \neq a^{-1}(0) \neq \mathbb{T}^{1}$ e a possuir apenas zeros de ordem finita, então para cada $f \in \mathcal{E}$ que for flat em $a^{-1}(0) \subset \mathbb{T}^{2}$ existirá $u \in \mathcal{C}^{\infty}\left(\mathbb{T}^{2}\right)$ tal que $\mathrm{L} u=f$.

Prova: Para cada $u \in \mathcal{C}^{\infty}\left(\mathbb{T}^{2}\right)$, a série parcial de Fourier na variável $t$ e a continuidade do operador $\mathrm{L}$ fornecem $\mathrm{L} u=f$ se, e somente se,

$$
a(x) \hat{u}_{k}^{\prime}(x)+i k \hat{u}_{k}(x)=\hat{f}_{k}(x)
$$

para todo $x \in \mathbb{T}^{1}$ e $k \in \mathbb{Z}$. Sendo assim, obter $u \in \mathcal{C}^{\infty}\left(\mathbb{T}^{2}\right)$ tal que $\mathrm{L} u=f$ é equivalente a obter uma sequência de funções de decrescimento rápido em $\mathcal{C}^{\infty}\left(\mathbb{T}^{1}\right)$ que satisfaz $(.7)$.

No caso em que $k=0$, devemos resolver a equação $a(x) \hat{u}_{0}^{\prime}(x)=\hat{f}_{0}(x)$, onde $x \in \mathbb{T}^{1}$. Segue da Proposição (2.47) que $\hat{f}_{0}$ é flat em $a^{-1}(0) \subset \mathbb{T}^{1}$ e como a possui apenas zeros de ordem finita em $\mathbb{T}^{1}$, segue do Corolário (2.33) que existe $v_{0} \in \mathcal{C}^{\infty}\left(\mathbb{T}^{1}\right)$ tal que $a(x) v_{0}(x)=\hat{f}_{0}(x)$. Falta concluir que $v_{0}$ é uma função que possui primitiva, o que ocorre se, e somente se, $\left\langle 1, v_{0}\right\rangle=0$. Pelo Teorema (2.42) existe $1 / a \in \mathcal{D}^{\prime}\left(\mathbb{T}^{1}\right)$ tal que $a(1 / a)=1$ em $\mathcal{D}^{\prime}\left(\mathbb{T}^{1}\right)$. Assim,

$$
\left\langle 1, v_{0}\right\rangle=\left\langle a(1 / a), v_{0}\right\rangle=\left\langle 1 / a, \hat{f}_{0}\right\rangle=\left\langle 1 / a,(2 \pi)^{-1}\left\langle 1_{t}, f(x, \cdot)\right\rangle\right\rangle=(2 \pi)^{-1}\left\langle(1 / a) \otimes 1_{t}, f\right\rangle
$$

e como $-{ }^{t} \mathrm{~L}\left((1 / a) \otimes 1_{t}\right)=(1 / a) \otimes \partial_{t} 1_{t}+\partial_{x}\left(a\left((1 / a) \otimes 1_{t}\right)\right)=\left(\partial_{x} 1_{x}\right) \otimes 1_{t}=0$, segue que $(1 / a) \otimes 1_{t} \in \operatorname{ker}^{t} \mathrm{~L}$. Portanto, a hipótese $f \in \mathcal{E}=\left(\operatorname{ker}^{t} \mathrm{~L}\right)^{\circ}$ implica que $\left\langle 1, v_{0}\right\rangle=$ $(2 \pi)^{-1}\left\langle(1 / a) \otimes 1_{t}, f\right\rangle=0$. Então existe $\hat{u}_{0} \in \mathcal{C}^{\infty}\left(\mathbb{T}^{1}\right)$ tal que $\hat{u}_{0}^{\prime}=v_{0}$, logo $\hat{u}_{0}$ satisfaz $a(x) \hat{u}_{0}^{\prime}(x)=a(x) v_{0}(x)=\hat{f}_{0}(x), x \in \mathbb{T}^{1}$.

Consideremos agora o caso em que $k \in \mathbb{Z} \backslash\{0\}$. Utilizando as propriedades do isomorfismo entre $\mathcal{C}^{\infty}\left(\mathbb{T}^{1}\right)$ e $\mathcal{P}_{2 \pi}(\mathbb{R})$, segue que resolver a equação $(.7)$ em $\mathbb{T}^{1}$ é equivalente a resolver a equação associada em $\mathcal{P}_{2 \pi}(\mathbb{R})$. Para facilitar a notação iremos omitir o isomorfismo $\Lambda$.

Escolha $x_{1} \in a^{-1}(0)$, denote o conjunto $a^{-1}(0) \cap\left[x_{1}, x_{1}+2 \pi\right)$ por $\left\{x_{1}<\cdots<x_{N}\right\}$ e defina $x_{N+1}=x_{1}+2 \pi$. 
Com base nas ideias que antecedem a Proposição (3.9), fixe $y_{j} \in\left(x_{j}, x_{j+1}\right)$, para $j=$ $1, \ldots, N$ e defina $A_{j}(x)=\int_{y_{j}}^{x} a^{-1}(z) d z$, para $x \in\left(x_{j}, x_{j+1}\right)$. Então $A_{j} \in \mathcal{C}^{\infty}\left(\left(x_{j}, x_{j+1}\right)\right)$, $\operatorname{logo} \exp \left\{i k A_{j}\right\} \in \mathcal{C}^{\infty}\left(\left(x_{j}, x_{j+1}\right)\right)$ e é limitada. Das proposições (2.47) e (2.8) segue que, para cada $k \in \mathbb{Z} \backslash\{0\}$, existe $h_{k} \in \mathcal{P}_{2 \pi}(\mathbb{R})$, flat em $a^{-1}(0)$ e tal que $h_{k}=\hat{f}_{k} / a$ em $\left(x_{j}, x_{j+1}\right)$, para $j=1, \ldots, N$. Da Proposição (3.9) resulta que cada função

$$
g_{k}^{j}(x)=\left\{\begin{aligned}
\exp \left\{i k A_{j}(x)\right\} \hat{f}_{k}(x) / a(x), & \text { se } \quad x \in\left(x_{j}, x_{j+1}\right) \\
0, & \text { se } \quad x=x_{j} \text { ou } x=x_{j+1}
\end{aligned}\right.
$$

pertence a $\mathcal{C}^{\infty}\left(\left(x_{j}, x_{j+1}\right)\right)$ e $\left(g_{k}^{j}\right)^{(n)}\left(x_{j}^{+}\right)=0=\left(g_{k}^{j}\right)^{(n)}\left(x_{j+1}^{-}\right)$, para todo $n \in \mathbb{Z}_{+}$. Sendo assim, se definirmos $x_{0}=x_{N}-2 \pi, x_{N+2}=x_{2}+2 \pi$ e se mostrarmos que

$$
\int_{x_{j}}^{x_{j+1}} g_{k}^{j}(x) d x=0
$$

para todo $j=1, \ldots, N$ e $k \in \mathbb{Z} \backslash\{0\}$, então a função

$$
G_{k}^{j}(x)=\left\{\begin{array}{rll}
0, & \text { se } & x \in\left[x_{j+1}, x_{j+2}\right) \\
\int_{x_{j}}^{x} g_{k}^{j}(y) d y, & \text { se } & x \in\left(x_{j}, x_{j+1}\right) \\
0, & \text { se } & x \in\left(x_{j-1}, x_{j}\right]
\end{array}\right.
$$

será infinitamente diferenciável em uma vizinhança de $\left[x_{j}, x_{j+1}\right]$ e flat em $\left\{x_{j}, x_{j+1}\right\}$. Assim, supondo valer (.8), segue da Proposição (3.9) que

$$
\hat{u_{k}}(x)=\left\{\begin{array}{rll}
\exp \left\{-i k A_{j}(x)\right\} \int_{x_{j}}^{x} g_{k}^{j}(y) d y, & \text { para } & x \in\left(x_{j}, x_{j+1}\right) \text { e } j=1, \ldots, N \\
0, & \text { se } & x \in\left\{x_{1}<\cdots<x_{N+1}\right\}
\end{array}\right.
$$

pertence a $\mathcal{C}^{\infty}\left(\left(x_{1}, x_{N+1}\right)\right)$, é flat em $\left\{x_{2}, \ldots, x_{N}\right\}$ e ${\hat{u_{k}}}^{(n)}\left(x_{1}^{+}\right)=0={\hat{u_{k}}}^{(n)}\left(x_{N+1}^{-}\right)$, para todo $n \in \mathbb{Z}_{+}$. Mais ainda, $\hat{u}_{k}$ satisfaz a equação (.7) em $\left[x_{1}, x_{N+1}\right]$. Tomando as extensões $2 \pi$-periódicas obtemos uma sequência de funções $\left(\hat{u}_{k}\right)_{k \in \mathbb{Z}}$ em $\mathcal{C}^{\infty}\left(\mathbb{T}^{1}\right)$ que são flat em $a^{-1}(0)$ e que satisfazem (.7). Se mostrarmos que $\left(\hat{u}_{k}\right)_{k \in \mathbb{Z}}$ é uma sequência de funções rapidamente decrescente, então $\left(\hat{u}_{k}\right)_{k \in \mathbb{Z}}$ definirá uma função $u \in \mathcal{C}^{\infty}\left(\mathbb{T}^{2}\right)$, a qual será dada por $u(x, t)=\sum_{k \in \mathbb{Z}} \hat{u}_{k}(x) e^{i k t}$ e satisfará $\mathbf{L} u=f$. Observe que $u$ também será flat em $a^{-1}(0) \subset \mathbb{T}^{2}$

Provaremos que $\left(\hat{u}_{k}\right)_{k \in \mathbb{Z}}$ decresce rápido na Proposição (3.11). Para concluir a demonstração falta provar as identidades (.8) para cada $j=1, \ldots, N$ e $k \in \mathbb{Z} \backslash\{0\}$, as quais serão uma consequência da hipótese de que $f \in \mathcal{E}=\left(\operatorname{ker}^{t} \mathrm{~L}\right)^{\circ}$. O que faremos é encontrar certas distribuições em $\operatorname{ker}^{t} \mathrm{~L}$ que implicarão as identidades (.8). 
A definição de operador transposto fornece que $-{ }^{t} \mathbf{L}=\partial_{t}+\partial_{x}(a \cdot)$ e a série parcial de Fourier na variável $t$ fornece que $v \in \operatorname{ker}^{t} \mathrm{~L}$ se, e somente se, $\hat{v}_{k} \otimes e_{k} \in \operatorname{ker}^{t} \mathrm{~L}$ para todo $k \in \mathbb{Z}$, o que ocorre se, e somente se, $-{ }^{t} \mathrm{~L}_{k}\left(\hat{v}_{k}\right) \doteq\left(a \hat{v}_{k}\right)^{\prime}+i k \hat{v}_{k}=0$, para todo $k \in \mathbb{Z}$. Assim sendo, cada distribuição $v_{k} \in \operatorname{ker}{ }^{t} L_{k}$ define uma distribuição $v=v_{k} \otimes e_{k}=$ $\hat{v}_{k} \otimes e_{k} \in \operatorname{ker}^{t} \mathrm{~L}, \log 0=\langle v, f\rangle=2 \pi\left\langle v_{k}, \hat{f}_{-k}\right\rangle$, isto é, $\left\langle v_{k}, \hat{f}_{-k}\right\rangle=0$.

Relembrando que

$$
\int_{x_{j}}^{x_{j+1}} g_{k}^{j}(x) d x=\int_{x_{j}}^{x_{j+1}} \exp \left\{i k A_{j}(x)\right\} \hat{f}_{k}(x) / a(x) d x ;
$$

fixe $j \in\{1, \ldots, N\}$ e para cada $k \in \mathbb{Z} \backslash\{0\}$ defina

$$
\psi_{k}(x)=\left\{\begin{array}{rll}
\exp \left\{-i k A_{j}(x)\right\}, & \text { se } & x \in\left(x_{j}, x_{j+1}\right) \\
0, & \text { se } & x \in \mathbb{T}^{1} \backslash\left(x_{j}, x_{j+1}\right) .
\end{array}\right.
$$

Desta maneira obtemos uma distribuição $\psi_{k} \in L^{\infty}\left(\mathbb{T}^{1}\right) \subset \mathcal{D}^{\prime}\left(\mathbb{T}^{1}\right)$. Como a ordem de anulamento dos zeros de $a$ é finita, segue do Teorema (2.42) que é possível dividir a distribuição $\psi_{k}$ pela função $a$. Mais ainda, uma análise mais cuidadosa permite obter tal divisão $\psi_{k} / a \in \mathcal{D}^{\prime}\left(\mathbb{T}^{1}\right)$ de maneira que $S\left(\psi_{k} / a\right) \subset S\left(\psi_{k}\right)$. Quando a função a possui apenas um ou dois zeros, então $a^{-1}(0) \subset S\left(\psi_{k}\right)$ e tal afirmação resulta da Observação (2.43). Quando a função a possui mais de dois zeros, tal afirmação é uma consequência da existência de partições da unidade em $\mathbb{T}^{1}$ (para partições da unidade em variedades citamos a referência 6)

Definindo $w_{k} \doteq{ }^{t} \mathrm{~L}_{k}\left(\psi_{k} / a\right)=\psi_{k}^{\prime}+i k \psi_{k} / a$ segue que $S\left(w_{k}\right) \subset S\left(\psi_{k}\right) \cup S\left(\psi_{k} / a\right) \subset$ $S\left(\psi_{k}\right)=\left[x_{j}, x_{j+1}\right] \subset \mathbb{T}^{1}$. Para cada $\phi \in \mathcal{C}^{\infty}\left(\mathbb{T}^{1}\right) \operatorname{com} S(\phi) \subset\left(x_{j}, x_{j+1}\right)$, temos $\left\langle w_{k}, \phi\right\rangle=$ $\left\langle\psi_{k}^{\prime}, \phi\right\rangle+i k\left\langle\psi_{k} / a, \phi\right\rangle=-\left\langle\psi_{k}, \phi^{\prime}\right\rangle+i k\left\langle\psi_{k} / a, \phi\right\rangle$. Como a função a não se anula em $\left(x_{j}, x_{j+1}\right)$, segue que $i k\left\langle\psi_{k} / a, \phi\right\rangle=i k\left\langle a\left(\psi_{k} / a\right), \phi / a\right\rangle=i k\left\langle\psi_{k}, \phi / a\right\rangle$. Utilizando integração por partes e o fato de que $\phi \in \mathcal{C}_{c}^{\infty}\left(\left(x_{j}, x_{j+1}\right)\right)$, obtemos $\left\langle\psi_{k}, \phi^{\prime}\right\rangle=i k\left\langle\psi_{k}, \phi / a\right\rangle$. Portanto, $\left\langle w_{k}, \phi\right\rangle=0$ para toda $\phi$ suportada em $\left(x_{j}, x_{j+1}\right)$, $\operatorname{logo} S\left(w_{k}\right) \subset\left\{x_{j}, x_{j+1}\right\}$.

Segue do Corolário (2.39) que existe uma distribuição $\nu_{k}$ satisfazendo ${ }^{t} \mathrm{~L}_{k}\left(\nu_{k}\right)=w_{k}$ e $S\left(\nu_{k}\right) \subset\left\{x_{j}, x_{j+1}\right\}$. Então $\hat{v}_{k} \doteq \psi_{k} / a-\nu_{k}$ pertence ao núcleo de ${ }^{t} \mathrm{~L}_{k}, \operatorname{logo} 0=$ $\left\langle\hat{v}_{k}, \hat{f}_{-k}\right\rangle=\left\langle\psi_{k} / a, \hat{f}_{-k}\right\rangle-\left\langle\nu_{k}, \hat{f}_{-k}\right\rangle$. Como $\hat{f}_{-k}$ é flat em $a^{-1}(0)$, segue da Observação (2.40) que $\left\langle\nu_{k}, \hat{f}_{-k}\right\rangle=0$. Assim, segue do Corolário (2.33) que $0=\left\langle\psi_{k} / a, \hat{f}_{-k}\right\rangle=$ $\left\langle a\left(\psi_{k} / a\right), \hat{f}_{-k} / a\right\rangle=\left\langle\psi_{k}, \hat{f}_{-k} / a\right\rangle=\int_{x_{j}}^{x_{j+1}} \exp \left\{-i k A_{j}(x)\right\} \hat{f}_{-k}(x) / a(x) d x=\int_{x_{j}}^{x_{j+1}} g_{-k}^{j}(x) d x$.

O resultado a seguir foi utilizado na demonstração do Teorema (3.10). 
3.11 Proposição. A sequência $\left(\hat{u}_{k}\right)_{k \in \mathbb{Z}} \subset \mathcal{C}^{\infty}\left(\mathbb{T}^{1}\right)$, definida na demonstração do Teorema (3.10), é uma sequência rapidamente decrescente.

Prova: Para $x \in\left(x_{j}, x_{j+1}\right)$ e $n \in \mathbb{Z}_{+}$temos $\partial^{n} \hat{u}_{k}(x)=B_{n}(x)+D_{n}(x)$, onde

$$
B_{n}(x) \doteq \partial^{n}\left(\exp \left\{-i k A_{j}(x)\right\}\right) \int_{x_{j}}^{x} g_{k}^{j}(y) d y
$$

$D_{0} \equiv 0$ e para $n \geq 1 D_{n}(x) \doteq$

$$
\sum_{l=0}^{n-1} \sum_{m=0}^{n-1-l}\left(\begin{array}{c}
n \\
l
\end{array}\right)\left(\begin{array}{c}
n-1-l \\
m
\end{array}\right) \partial^{l}\left(\exp \left\{-i k A_{j}(x)\right\}\right) \partial^{m}\left(\exp \left\{i k A_{j}(x)\right\}\right) \partial^{n-1-l-m}\left(\hat{f}_{k} / a\right)(x)
$$

Defina $g(x) \doteq i k A_{j}(x)$. Para cada $r, s \in \mathbb{N}$, denotaremos por $P_{s}\left(g^{(1)}(x), \ldots, g^{(r)}(x)\right)$ um polinômio da forma

$$
\sum_{\alpha \in \mathbb{Z}_{+}^{r},|\alpha| \leq s} c_{\alpha}\left(g^{(1)}(x), \ldots, g^{(r)}(x)\right)^{\alpha}
$$

e denotaremos por $P_{s}\left(a(x), a^{(1)}(x), \ldots, a^{(r)}(x)\right)$ um polinômio da forma

$$
\sum_{\alpha \in \mathbb{Z}_{+}^{r+1},|\alpha| \leq s} c_{\alpha}\left(a(x), a^{(1)}(x), \ldots, a^{(r)}(x)\right)^{\alpha}
$$

Verifica-se que $\partial^{l}\left(\exp \left\{-i k A_{j}(x)\right\}\right)=P_{s_{l}}\left(g^{(1)}(x), \ldots, g^{(l)}(x)\right) \exp \left\{-i k A_{j}(x)\right\}$ e que $\partial^{m}\left(\exp \left\{i k A_{j}(x)\right\}\right)=P_{s_{m}}\left(g^{(1)}(x), \ldots, g^{(m)}(x)\right) \exp \left\{i k A_{j}(x)\right\}$. Assim,

$$
\partial^{l}\left(\exp \left\{-i k A_{j}(x)\right\}\right) \partial^{m}\left(\exp \left\{i k A_{j}(x)\right\}\right)=P_{s_{l, m}}\left(g^{(1)}(x), \ldots, g^{\left(M_{l, m}\right)}(x)\right)
$$

onde $M_{l, m} \doteq \max \{l, m\}$.

Como $g^{\prime}(x)=i k\left(A_{j}\right)^{\prime}(x)=i k a^{-1}(x)$ e $g^{(n)}(x)=i k\left(A_{j}\right)^{(n)}(x)=i k \partial^{n-1}(1 / a)(x)=$ $i k a(x)^{-2^{n-1}} P_{s_{n}}\left(a(x), a^{(1)}(x), \ldots, a^{(n-1)}(x)\right)$, se $n>1$, obtemos a identidade

$$
\begin{gathered}
P_{s_{l, m}}\left(g^{(1)}(x), \ldots, g^{\left(M_{l, m}\right)}(x)\right)= \\
\sum_{\alpha \in \mathbb{Z}_{+}^{M_{l, m},|\alpha| \leq s_{l, m}}} c_{\alpha}(i k)^{|\alpha|} a(x)^{-\sum_{p=1}^{M_{l, m}} \alpha_{p} 2^{(p-1)}} P_{s_{\alpha}}\left(a(x), a^{\prime}(x), \ldots, a^{\left(M_{l, m}\right)}(x)\right) .
\end{gathered}
$$

Definindo $\phi(x, t) \doteq f(x, t) / a(x)$, se $(x, t) \in \mathbb{T}^{2} \backslash a^{-1}(0)$ e $\phi(x, t)=0$, se $(x, t) \in a^{-1}(0)$, segue do Corolário (2.34) que $\phi \in \mathcal{C}^{\infty}\left(\mathbb{T}^{2}\right)$ é flat em $a^{-1}(0) \subset \mathbb{T}^{2}$ e cálculos diretos mostram que $\hat{\phi}_{k}=\hat{f}_{k} / a \in \mathcal{C}^{\infty}\left(\mathbb{T}^{1}\right)$, para todo $k \in \mathbb{Z}$. 
Assim, dado $M \in \mathbb{Z}_{+}$temos $|k|^{M}\left|D_{n}(x)\right| \leq$

$$
(n !)^{2} \sum_{l=0}^{n-1} \sum_{m=0}^{n-1-l} \sum_{\substack{\alpha \in \mathbb{Z}_{+}^{M_{l, m}} \\|\alpha| \leq s_{l, m}}} C_{\alpha}\left|k^{|\alpha|+M} a(x)^{-\sum_{p=1}^{M_{l, m}} \alpha_{p} 2^{(p-1)}}\left(\partial^{n-1-l-m} \hat{\phi}_{k}\right)(x)\right|,
$$

onde cada constante $C_{\alpha}$ absorve a constante $c_{\alpha}$ e a norma $\left\|P_{s_{\alpha}}\left(a(x), \ldots, a^{\left(M_{l, m}\right)}(x)\right)\right\|_{\infty}$, logo $C_{\alpha}$ não depende de $x$ e também não depende de $k$.

Como

$$
\begin{gathered}
\left|k^{|\alpha|+M}\left(\partial^{n-1-l-m} \hat{\phi}_{k}\right)(x)\right|=(2 \pi)^{-1}\left|\int_{0}^{2 \pi}\left(\partial^{(n-1-l-m, 0)} \phi\right)(x, t) k^{|\alpha|+M} e^{-i k t} d t\right| \leq \\
(2 \pi)^{-1} \int_{0}^{2 \pi}\left|\left(\partial^{(n-1-l-m,|\alpha|+M)} \phi\right)(x, t)\right| d t,
\end{gathered}
$$

temos

$$
\begin{gathered}
\left|k^{|\alpha|+M} a(x)^{-\sum_{p=1}^{M_{l, m}} \alpha_{p} 2^{(p-1)}}\left(\partial^{n-1-l-m} \hat{\phi}_{k}\right)(x)\right| \leq \\
(2 \pi)^{-1} \int_{0}^{2 \pi}\left|a(x)^{-\sum_{p=1}^{M_{l, m}} \alpha_{p} 2^{(p-1)}}\left(\partial^{(n-1-l-m,|\alpha|+M)} \phi\right)(x, t)\right| d t \leq \\
\left\|a(x)^{-\sum_{p=1}^{M_{l, m}} \alpha_{p} 2^{(p-1)}}\left(\partial^{(n-1-l-m,|\alpha|+M)} \phi\right)(x, t)\right\|_{\infty},
\end{gathered}
$$

a qual existe pois $\phi \in \mathcal{C}^{\infty}\left(\mathbb{T}^{2}\right)$ é flat em $a^{-1}(0) \subset \mathbb{T}^{2}$ e $a \in \mathcal{C}^{\infty}\left(\mathbb{T}^{1}\right)$ possui apenas zeros de ordem finita.

Portanto, dados $n, M \in \mathbb{Z}_{+}$, existe $C_{1}>0$ tal que $|k|^{M}\left|D_{n}(x)\right| \leq C_{1}$, para todo $x \in\left(x_{j}, x_{j+1}\right)$ e $k \in \mathbb{Z} \backslash\{0\}$.

Utilizando argumentos similares, obtemos $C_{2}>0$ tal que $|k|^{M}\left|B_{n}(x)\right| \leq C_{2}$, para todo $x \in\left(x_{j}, x_{j+1}\right)$ e $k \in \mathbb{Z} \backslash\{0\}$.

Em suma, dados $n, M \in \mathbb{Z}_{+}$, existe $C>0$ satisfazendo $|k|^{M}\left|\partial^{n} \hat{u}_{k}(x)\right| \leq C$, para todo $x \in \mathbb{T}^{1}$ e $k \in \mathbb{Z} \backslash\{0\}$, o que mostra que a sequência $\left(\hat{u}_{k}\right)_{k \in \mathbb{Z}} \subset \mathcal{C}^{\infty}\left(\mathbb{T}^{1}\right)$ é rapidamente decrescente.

Os teoremas $(3.5)$ e (3.10) provam que quando $\emptyset \neq a^{-1}(0) \neq \mathbb{T}^{1}$, a hipótese de que $a^{-1}(0)$ contém apenas zeros de ordem finita é uma condição suficiente para que $\mathrm{LC}^{\infty}\left(\mathbb{T}^{2}\right) \supset \mathcal{E}=\left(\operatorname{ker}^{t} \mathrm{~L}\right)^{\circ}$, ou seja, para que $\mathrm{LC} \mathcal{C}^{\infty}\left(\mathbb{T}^{2}\right)$ seja um subespaço fechado de $\mathcal{C}^{\infty}\left(\mathbb{T}^{2}\right)$

Utilizando as mesmas técnicas presentes na demonstração do Teorema (3.10), conseguimos verificar que tal resultado também é válido para os operadores do tipo $\mathrm{P}$. 
3.12 Teorema. Se $\emptyset \neq a^{-1}(0) \neq \mathbb{T}^{1}$ e se a possuir apenas zeros de ordem finita, então para cada $f \in \mathcal{G}$ que for flat em $a^{-1}(0) \subset \mathbb{T}^{2}$, existirá $u \in \mathcal{C}^{\infty}\left(\mathbb{T}^{2}\right)$ tal que $\mathrm{P} u=f$.

Prova: Utilizando série parcial de Fourier na variável $t$ segue que existe $u \in \mathcal{C}^{\infty}\left(\mathbb{T}^{2}\right)$ satisfazendo $\mathrm{P} u=f$ se, e somente se,

$$
\left(a \hat{u}_{k}\right)^{\prime}(x)+i k \hat{u}_{k}(x)=\hat{f}_{k}(x)
$$

para todo $x \in \mathbb{T}^{1}$ e $k \in \mathbb{Z}$.

No caso em que $k=0$, devemos resolver a equação $\left(a \hat{u}_{0}\right)^{\prime}(x)=\hat{f}_{0}(x)$ em $\mathbb{T}^{1}$. Sabemos que $\hat{f}_{0} \in \mathcal{C}^{\infty}\left(\mathbb{T}^{1}\right)$ possui uma primitiva se, e somente se,

$$
(2 \pi)^{-1} \int_{0}^{2 \pi} \int_{0}^{2 \pi} f(x, t) d t d x=\int_{0}^{2 \pi} \hat{f}_{0}(x) d x=0 .
$$

Por hipótese, $f \in \operatorname{ker}^{t} \mathrm{P}$ e $-{ }^{t} \mathrm{P}=\mathrm{L}$. Assim, $1_{x} \otimes 1_{t} \in \operatorname{ker}{ }^{t} \mathrm{P}$ e então $0=\left\langle 1_{x} \otimes 1_{t}, f\right\rangle=$ $\left\langle 1_{x},\left\langle 1_{t}, f(x, \cdot)\right\rangle\right\rangle=2 \pi\left\langle 1_{x}, \hat{f}_{0}\right\rangle=2 \pi \int_{0}^{2 \pi} \hat{f}_{0}(x) d x$. Portanto, existe $v_{0} \in \mathcal{C}^{\infty}\left(\mathbb{T}^{1}\right)$ tal que $v_{0}^{\prime}=\hat{f}_{0}$. Como a é uma função que possui apenas zeros de ordem finita, segue do Teorema (2.42) que existe $\hat{u}_{0}=v_{0} / a \in \mathcal{C}^{\infty}\left(\mathbb{T}^{1}\right)$ tal que $\left(a \hat{u}_{0}\right)^{\prime}=v_{0}^{\prime}=\hat{f}_{0}$.

O próximo passo é resolver (.9) para $k \in \mathbb{Z} \backslash\{0\}$. Fazendo as mesmas considerações que foram feitas na demonstração do Teorema (3.10), tome $x_{1} \in a^{-1}(0)$, denote o conjunto $a^{-1}(0) \cap\left[x_{1}, x_{1}+2 \pi\right)$ por $\left\{x_{1}<x_{2}<\cdots<x_{N}\right\}$ e defina $x_{N+1}=x_{1}+2 \pi$. Com base nas ideias que antecedem a Proposição (3.9), se $y_{j}, z_{j} \in\left(x_{j}, x_{j+1}\right)$, então utilizando fator integrante pode-se verificar que a expressão

$$
\begin{gathered}
c \exp \left\{-i k \int_{y_{j}}^{x} a^{-1}(y) d y\right\}+ \\
\exp \left\{-i k \int_{y_{j}}^{x} a^{-1}(y) d y\right\} \int_{z_{j}}^{x} \hat{f}_{k}(y) \exp \left\{i k \int_{y_{j}}^{y} a^{-1}(z) d z\right\} d y
\end{gathered}
$$

satisfaz a equação $v_{k}^{\prime}(x)+i k v_{k}(x) / a(x)=\hat{f}_{k}(x)$ no intervalo $\left(x_{j}, x_{j+1}\right)$, qualquer que seja $c \in \mathbb{C}$.

Seguindo as ideias acima, escolha $y_{j} \in\left(x_{j}, x_{j+1}\right), j=1, \ldots, N$ e defina $A_{j}(x)=$ $\int_{y_{j}}^{x} a^{-1}(z) d z$, para $x \in\left(x_{j}, x_{j+1}\right)$. Então $A_{j} \in \mathcal{C}^{\infty}\left(\left(x_{j}, x_{j+1}\right)\right)$ e para cada $k \in \mathbb{Z} \backslash\{0\}$ a função $\exp \left\{i k A_{j}\right\} \in \mathcal{C}^{\infty}\left(\left(x_{j}, x_{j+1}\right)\right)$ e é limitada. Da Proposição (3.9) resulta que cada função

$$
g_{k}^{j}(x)=\left\{\begin{aligned}
\exp \left\{i k A_{j}(x)\right\} \hat{f}_{k}(x), & \text { se } x \in\left(x_{j}, x_{j+1}\right) \\
0, & \text { se } x=x_{j} \text { ou } x=x_{j+1}
\end{aligned}\right.
$$


pertence a $\mathcal{C}^{\infty}\left(\left(x_{j}, x_{j+1}\right)\right)$ e $\left(g_{k}^{j}\right)^{(n)}\left(x_{j}^{+}\right)=0=\left(g_{k}^{j}\right)^{(n)}\left(x_{j+1}^{-}\right)$, para todo $n \in \mathbb{Z}_{+}$. Sendo assim, se definirmos $x_{0}=x_{N}-2 \pi, x_{N+2}=x_{2}+2 \pi$ e se mostrarmos que

$$
\int_{x_{j}}^{x_{j+1}} g_{k}^{j}(x) d x=0
$$

para todo $j=1, \ldots, N$ e $k \in \mathbb{Z} \backslash\{0\}$, então a função

$$
G_{k}^{j}(x)=\left\{\begin{array}{rll}
0, & \text { se } & x \in\left[x_{j+1}, x_{j+2}\right) \\
\int_{x_{j}}^{x} g_{k}^{j}(y) d y, & \text { se } & x \in\left(x_{j}, x_{j+1}\right) \\
0, & \text { se } & x \in\left(x_{j-1}, x_{j}\right]
\end{array}\right.
$$

será infinitamente diferenciável em uma vizinhança de $\left[x_{j}, x_{j+1}\right]$ e flat em $\left\{x_{j}, x_{j+1}\right\}$. Assim, supondo valer (.10), segue da Proposição (3.9) que

$$
v_{k}(x)=\left\{\begin{array}{rll}
\exp \left\{-i k A_{j}(x)\right\} \int_{x_{j}}^{x} g_{k}^{j}(y) d y, & \text { para } & x \in\left(x_{j}, x_{j+1}\right) \text { e } j=1, \ldots, N \\
0, & \text { se } \quad x \in\left\{x_{1}<\cdots<x_{N+1}\right\}
\end{array}\right.
$$

pertence a $\mathcal{C}^{\infty}\left(\left(x_{1}, x_{N+1}\right)\right)$, é flat em $\left\{x_{2}, \ldots, x_{N}\right\}$ e $v_{k}^{(n)}\left(x_{1}^{+}\right)=0=v_{k}^{(n)}\left(x_{N+1}^{-}\right)$, para todo $n \in \mathbb{Z}_{+}$. Mais ainda, $v_{k}$ satisfaz a equação $v_{k}^{\prime}(x)+i k v_{k}(x) / a(x)=\hat{f}_{k}(x)$ em $\left(x_{1}, x_{N+1}\right)$. Tomando as extensões $2 \pi$-periódicas obtemos uma sequência de funções $\left(v_{k}\right)_{k \in \mathbb{Z}}$ em $\mathcal{C}^{\infty}\left(\mathbb{T}^{1}\right)$ que são flat em $a^{-1}(0)$ e que satisfazem $v_{k}^{\prime}(x)+i k v_{k}(x) / a(x)=\hat{f}_{k}(x)$. Observe que como a função a possui apenas zeros de ordem finita, existe $v_{k} / a \in \mathcal{C}^{\infty}\left(\mathbb{T}^{1}\right)$ flat em $a^{-1}(0)$. Então $\hat{u}_{k}(x)=v_{k}(x) / a(x)=$

$$
\left\{\begin{array}{rll}
\exp \left\{-i k A_{j}(x)\right\} a^{-1}(x) \int_{x_{j}}^{x} g_{k}^{j}(y) d y, & \text { para } & x \in\left(x_{j}, x_{j+1}\right) \subset \mathbb{T}^{1} \text { e } j=1, \ldots, N \\
0, & \text { se } & x \in a^{-1}(0)
\end{array}\right.
$$

é uma função em $\mathcal{C}^{\infty}\left(\mathbb{T}^{1}\right)$, flat em $a^{-1}(0)$ e que satisfaz a equação (.9). Argumentos análogos aos utilizados na demonstração da Proposição (3.11) demonstram que a sequência $\left(\hat{u}_{k}\right)_{k \in \mathbb{Z}} \subset \mathcal{C}^{\infty}\left(\mathbb{T}^{1}\right)$ é rapidamente decrescente, logo $\left(\hat{u}_{k}\right)_{k \in \mathbb{Z}}$ define uma função $u \in \mathcal{C}^{\infty}\left(\mathbb{T}^{2}\right)$ que satisfaz $\mathrm{P} u=f$.

Para finalizar esta demonstração resta provar as identidades (.10), para cada $j=$ $1, \ldots, N$ e $k \in \mathbb{Z} \backslash\{0\}$. Utilizaremos a mesma técnica que foi empregada na demonstração para a classe de operadores do tipo L. Calculando o operador transposto obtemos $-{ }^{t} \mathrm{P}=$ $\partial_{t}+a(x) \partial_{x}$ e a série parcial de Fourier na variável $t$ fornece que $v \in \operatorname{ker}^{t} \mathrm{P}$ se, e somente se, $\hat{v}_{k} \otimes e_{k} \in \operatorname{ker}{ }^{t} \mathrm{P}$ para todo $k \in \mathbb{Z}$, o que ocorre se, e somente se, $-{ }^{t} \mathrm{P}_{k}\left(\hat{v}_{k}\right) \doteq$ $a \hat{v}_{k}^{\prime}+i k \hat{v}_{k}=0$, para todo $k \in \mathbb{Z}$. Assim, cada distribuição $\nu_{k} \in \operatorname{ker}^{t} \mathrm{P}_{k}$ define uma 
distribuição $v=\nu_{k} \otimes e_{k}=\hat{v}_{k} \otimes e_{k} \in \operatorname{ker}^{t} \mathrm{P}, \operatorname{logo} 0=\langle v, f\rangle=2 \pi\left\langle\nu_{k}, \hat{f}_{-k}\right\rangle$, ou seja $\left\langle\nu_{k}, \hat{f}_{-k}\right\rangle=0$.

Relembrando que

$$
\int_{x_{j}}^{x_{j+1}} g_{k}^{j}(x) d x=\int_{x_{j}}^{x_{j+1}} \exp \left\{i k A_{j}(x)\right\} \hat{f}_{k}(x) d x
$$

fixe $j \in\{1, \ldots, N\}$ e para cada $k \in \mathbb{Z} \backslash\{0\}$ defina

$$
\psi_{k}(x)=\left\{\begin{array}{rll}
\exp \left\{-i k A_{j}(x)\right\}, & \text { se } & x \in\left(x_{j}, x_{j+1}\right) \\
0, & \text { se } & x \in \mathbb{T}^{1} \backslash\left(x_{j}, x_{j+1}\right) .
\end{array}\right.
$$

Desta maneira obtemos uma distribuição $\psi_{k} \in L^{\infty}\left(\mathbb{T}^{1}\right) \subset \mathcal{D}^{\prime}\left(\mathbb{T}^{1}\right) \operatorname{com} S\left(\psi_{k}\right) \subset\left[x_{j}, x_{j+1}\right]$. Então a distribuição $w_{k} \doteq{ }^{t} \mathrm{P}_{k}\left(\psi_{k}\right)=a \psi_{k}^{\prime}+i k \psi_{k}$ também satisfaz $S\left(w_{k}\right) \subset\left[x_{j}, x_{j+1}\right]$ e para toda $\phi \in \mathcal{C}^{\infty}\left(\mathbb{T}^{1}\right) \operatorname{com} S(\phi) \subset\left(x_{j}, x_{j+1}\right)$, temos $\left\langle a \psi_{k}^{\prime}, \phi\right\rangle=-i k\left\langle\psi_{k}, \phi\right\rangle$, assim, $w_{k}=0$ em $\left(x_{j}, x_{j+1}\right)$ e consequentemente $S\left(w_{k}\right) \subset\left\{x_{j}, x_{j+1}\right\}$. Segue do Corolário (2.39) que existe $h_{k} \in \mathcal{D}^{\prime}\left(\mathbb{T}^{1}\right)$ tal que ${ }^{t} \mathrm{P}_{k} h_{k}=w_{k}$ e $S\left(h_{k}\right) \subset\left\{x_{j}, x_{j+1}\right\}$. Definindo $\nu_{k} \doteq$ $\psi_{k}-h_{k}$ segue que $\nu_{k} \in \operatorname{ker}{ }^{t} \mathrm{P}_{k}, \operatorname{logo} 0=\left\langle\nu_{k}, \hat{f}_{-k},\right\rangle=\left\langle\psi_{k}, \hat{f}_{-k}\right\rangle-\left\langle h_{k}, \hat{f}_{-k}\right\rangle$. Como $\hat{f}_{-k}$ é flat em $a^{-1}(0)$, segue da Observação (2.40) que $\left\langle h_{k}, \hat{f}_{-k}\right\rangle=0, \operatorname{logo} 0=\left\langle\psi_{k}, \hat{f}_{-k}\right\rangle=$ $\int_{x_{j}}^{x_{j+1}} \exp \left\{-i k A_{j}(x)\right\} \hat{f}_{-k}(x) d x=\int_{x_{j}}^{x_{j+1}} g_{-k}^{j}(x) d x$.

Os teoremas $(3.7)$ e $(3.12)$ provam que quando $\emptyset \neq a^{-1}(0) \neq \mathbb{T}^{1}$, a hipótese de que $a^{-1}(0)$ contém apenas zeros de ordem finita é uma condição suficiente para que $\mathrm{PC}^{\infty}\left(\mathbb{T}^{2}\right) \supset \mathcal{G}=\left(\operatorname{ker}^{t} \mathrm{P}\right)^{\circ}$, ou seja, para que $\mathrm{PC}^{\infty}\left(\mathbb{T}^{2}\right)$ seja um subespaço fechado de $\mathcal{C}^{\infty}\left(\mathbb{T}^{2}\right)$

Finalizando essa seção mostraremos que quando $\emptyset \neq a^{-1}(0) \neq \mathbb{T}^{1}$, a condição de que $a^{-1}(0)$ contém apenas zeros de ordem finita é uma condição necessária para que a imagem de um campo vetorial do tipo $L$ seja um subespaço fechado de $\mathcal{C} \infty\left(\mathbb{T}^{2}\right)$. Para tanto, utilizaremos a seguinte característica de um campo vetorial do tipo $L$, dada pela proposição abaixo.

3.13 Proposição. Seja $\mathrm{L}_{0} \doteq a(x) d / d_{x}: \mathcal{C}^{\infty}\left(\mathbb{T}^{1}\right) \rightarrow \mathcal{C}^{\infty}\left(\mathbb{T}^{1}\right)$. Se a imagem de $\mathrm{L}$ for um subespaço fechado de $\mathcal{C}^{\infty}\left(\mathbb{T}^{2}\right)$, então a imagem de $\mathrm{L}_{0}$ será um subespaço fechado de $\mathcal{C}^{\infty}\left(\mathbb{T}^{1}\right)$.

Prova: Suponha que $\mathrm{L}_{0} \mathcal{C}^{\infty}\left(\mathbb{T}^{1}\right)$ não seja um subespaço fechado de $\mathcal{C}^{\infty}\left(\mathbb{T}^{1}\right)$. Segue da Proposição (1.2) que existe $f_{0} \in\left(\operatorname{ker}^{t} \mathrm{~L}_{0}\right)^{\circ} \backslash \mathrm{L}_{0} \mathcal{C}^{\infty}\left(\mathbb{T}^{1}\right)$. 
Definindo $f=f_{0} \otimes 1_{t} \in \mathcal{C}^{\infty}\left(\mathbb{T}^{2}\right)$ e utilizando a série parcial de Fourier na variável $t$ de uma distribuição $v \in \mathcal{D}^{\prime}\left(\mathbb{T}^{2}\right)$, temos

$$
\langle v, f\rangle=\left\langle\sum_{k \in \mathbb{Z}} \hat{v}_{k} \otimes e_{k}, f_{0} \otimes 1_{t}\right\rangle=\sum_{k \in \mathbb{Z}}\left\langle\hat{v}_{k} \otimes e_{k}, f_{0} \otimes 1_{t}\right\rangle=\left\langle 1_{t},\left\langle\hat{v}_{0}, f_{0}\right\rangle\right\rangle
$$

Agora, $v \in \operatorname{ker}^{t} \mathrm{~L}$ se, e somente se, $i k \hat{v}_{k}+\left(a \hat{v}_{k}\right)^{\prime}=0$, para todo $k \in \mathbb{Z}$. Em particular, $v \in \operatorname{ker}{ }^{t} \mathrm{~L}$ implica que $0=\left(a \hat{v}_{0}\right)^{\prime}=-{ }^{t} \mathrm{~L}_{0}\left(\hat{v}_{0}\right)$, ou seja, $\hat{v}_{0} \in \operatorname{ker}{ }^{t} \mathrm{~L}_{0}$. Portanto, $\langle v, f\rangle=$ $\left\langle 1_{t},\left\langle\hat{v}_{0}, f_{0}\right\rangle\right\rangle=\left\langle 1_{t}, 0\right\rangle=0$, para toda $v \in \operatorname{ker}^{t} \mathrm{~L}$, resultando que $f \in\left(\operatorname{ker}^{t} \mathrm{~L}\right)^{\circ}$. Para concluirmos que $\mathrm{LC} \mathcal{C}^{\infty}\left(\mathbb{T}^{2}\right)$ não é um subespaço fechado de $\mathcal{C}^{\infty}\left(\mathbb{T}^{2}\right)$, basta mostrarmos que $f \in\left(\operatorname{ker}^{t} \mathrm{~L}\right)^{\circ} \backslash \mathrm{LC} \mathcal{C}^{\infty}\left(\mathbb{T}^{2}\right)$. Suponha que exista $u \in \mathcal{C}^{\infty}\left(\mathbb{T}^{2}\right)$ tal que $\mathrm{L} u=f$. Tomando as séries parciais de Fourier na variável $t$ das funcões $f$ e $u$ temos $\mathrm{L} u=f$ se, e somente se, $i k \hat{u}_{k}+a \hat{u}_{k}^{\prime}=\hat{f}_{k}$, para todo $k \in \mathbb{Z}$. Em particular, $\mathrm{L}_{0}\left(\hat{u}_{0}\right)=a \hat{u}_{0}^{\prime}=\hat{f}_{0}=f_{0}$, ou seja, $f_{0} \in \mathrm{L}_{0} \mathcal{C}^{\infty}\left(\mathbb{T}^{1}\right)$, o que é um absurdo.

3.14 Teorema. Se $a^{-1}(0) \neq \mathbb{T}^{1}$ e existe um ponto em $\mathbb{T}^{1}$ onde a é flat, então o operador $\mathrm{L}_{0}$ não possui imagem fechada em $\mathcal{C}^{\infty}\left(\mathbb{T}^{1}\right)$.

Prova: Pela Proposição (1.2) basta provarmos que $\mathrm{L}_{0} \mathcal{C}^{\infty}\left(\mathbb{T}^{1}\right) \subsetneq\left(\text { ker }{ }^{t} \mathrm{~L}_{0}\right)^{\circ}$. Vamos então exibir uma função em $\left(\operatorname{ker}^{t} \mathrm{~L}_{0}\right)^{\circ} \backslash \mathrm{L}_{0} \mathcal{C}^{\infty}\left(\mathbb{T}^{1}\right)$.

Calculando o operador transposto de $\mathrm{L}_{0}$ obtemos $-{ }^{t} \mathrm{~L}_{0}=d / d_{x}(a \cdot)$. Se $v$ é uma distribuição que está no núcleo de ${ }^{t} \mathrm{~L}_{0}$, então $v$ satisfaz $(a v)^{\prime}=0$ e da Proposição (2.48) segue que $a v=c$ em $\mathcal{D}^{\prime}\left(\mathbb{T}^{1}\right)$, onde $c \in \mathbb{C}$ é uma constante. Segue do Teorema (2.44) que $c=0, \operatorname{logo} a v=0$ para toda $v \in \operatorname{ker}^{t} \mathrm{~L}_{0}$. Portanto, $\operatorname{ker}^{t} \mathrm{~L}_{0}=\left\{v \in \mathcal{D}^{\prime}\left(\mathbb{T}^{1}\right) ; a v=0\right\}$.

Denote por $\mathcal{F}$ o conjunto dos pontos em que a é flat. Como $\mathcal{F}$ é um subconjunto fechado, não vazio e $\mathcal{F} \neq \mathbb{T}^{1}$, segue da conexidade de $\mathbb{T}^{1}$ que $\partial \mathcal{F} \neq \emptyset$.

Fixe $x_{1} \in \partial \mathcal{F}$ e defina $g(x) \doteq 1-\cos \left(x-x_{1}\right) \in \mathcal{C}^{\infty}\left(\mathbb{T}^{1}\right)$. Observe que $g$ se anula apenas em $x_{1}$, o qual é um zero de ordem dois, e a é flat em $x_{1}$, pois $\mathcal{F}$ ser fechado implica que $\partial \mathcal{F} \subset \mathcal{F}$. Segue do Corolário (2.33) que existe $f \in \mathcal{C}^{\infty}\left(\mathbb{T}^{1}\right)$, flat em $x_{1}$ e tal que $a=f g$.

Mostraremos que $f \in\left(\operatorname{ker}{ }^{t} \mathrm{~L}_{0}\right)^{\circ}$. De fato, para cada $v \in \operatorname{ker}{ }^{t} \mathrm{~L}_{0}$ temos $0=a v=$ $g(f v)=0$ e pelo Teorema (2.41) segue que $f v=c_{0} \delta\left(x-x_{1}\right)+c_{1} \delta^{\prime}\left(x-x_{1}\right)$. Pelo Teorema (2.44) devemos ter $c_{0}=c_{1}=0$, já que $f$ é flat em $x_{1}$. Assim, $f v=0$ e portanto, $\langle v, f\rangle=0$, qualquer que seja $v \in \operatorname{ker}^{t} \mathrm{~L}_{0}$.

Por fim, mostraremos que não existe $u \in \mathcal{C}^{\infty}\left(\mathbb{T}^{1}\right)$ tal que $\mathrm{L}_{0} u=f$. Se existir, então $a u^{\prime}=f$. Como $x_{1} \in \partial \mathcal{F}$, toda vizinhança de $x_{1}$ possui um ponto onde a função $a$ não se anula. Assim, existe uma sequência $\left(y_{n}\right)_{n \in \mathbb{N}} \subset \mathbb{T}^{1}$ que converge para $x_{1}$ e satisfaz $a\left(y_{n}\right) \neq 0$, para todo $n \in \mathbb{N}$. Então $f\left(y_{n}\right) \neq 0$, para todo $n \in \mathbb{N}$ e $f\left(y_{n}\right)=a\left(y_{n}\right) u^{\prime}\left(y_{n}\right)=$ 
$f\left(y_{n}\right) g\left(y_{n}\right) u^{\prime}\left(y_{n}\right)$ implica que $1=g\left(y_{n}\right) u^{\prime}\left(y_{n}\right)$, para todo $n \in \mathbb{N}$. Pela continuidade das funções $g$ e $u^{\prime}$ segue que $1=g\left(x_{1}\right) u^{\prime}\left(x_{1}\right)=0$, uma contradição.

A Proposição (3.13) e o Teorema (3.14) provam que quando $\emptyset \neq a^{-1}(0) \neq \mathbb{T}^{1}$, a finitude da ordem dos zeros da função $a$ é uma condição necessária para que $L \mathcal{C}^{\infty}\left(\mathbb{T}^{2}\right)=$ $\mathcal{E} \supset\left(\operatorname{ker}^{t} \mathrm{~L}\right)^{\circ}$, ou seja, para que $\mathrm{L} \mathcal{C}^{\infty}\left(\mathbb{T}^{2}\right)$ seja um subespaço fechado de $\mathcal{C}^{\infty}\left(\mathbb{T}^{2}\right)$.

Em suma, os resultados desta seção demonstram o Teorema (3.3). Também demonstram o Teorema (3.4) nos casos em que $a^{-1}(0)=\mathbb{T}^{1}$ e $a^{-1}(0)=\emptyset$. No caso em que $\emptyset \neq a^{-1}(0) \neq \mathbb{T}^{1}$, ficou demonstrado apenas que $a^{-1}(0)$ conter somente zeros de ordem finita é uma condição suficiente para que $P \mathcal{C}^{\infty}\left(\mathbb{T}^{2}\right)$ seja um subespaço fechado de $\mathcal{C}^{\infty}\left(\mathbb{T}^{2}\right)$

Vale observar que a parte final da demonstração do Teorema (3.10) mostra que quando $\emptyset \neq a^{-1}(0) \neq \mathbb{T}^{1}$, o operador transposto ${ }^{t} \mathrm{~L}$ não será injetor e, portanto, $\mathrm{L}$ não será sobrejetor . Mais ainda, tal demonstração mostra que $\operatorname{ker}^{t} \mathrm{~L}$ será um subespaço de dimensão infinita e, consequentemente, a imagem de $L$ não será de codimensão finita quando $\emptyset \neq a^{-1}(0) \neq \mathbb{T}^{1}$ (veja os comentários após a Definição (1.2) de [4]). Por fim, analisando a parte final da demonstração do Teorema (3.12), verifica-se que as mesmas conclusões são válidas para os operadores do tipo $P$; isto é, se $\emptyset \neq a^{-1}(0) \neq \mathbb{T}^{1}$, então nenhum operador do tipo $\mathrm{P}$ será fortemente resolúvel, tampouco sobrejetor.

\section{Resolubilidade global no espaço de distribuições}

Consideremos agora os operadores do tipo $L$ agindo no espaço de distribuições $\mathcal{D}^{\prime}\left(\mathbb{T}^{2}\right)$. O objetivo é caracterizar a resolubilidade global de tais operadores demonstrando o Teorema (3.1), o qual reescrevemos abaixo.

3.15 Teorema. Para um operador do tipo $\mathbf{L}=\partial_{t}+a(x) \partial_{x}$, as seguintes propriedades são equivalentes:

(i) $\mathrm{LD}^{\prime}\left(\mathbb{T}^{2}\right) \supset\left(\operatorname{ker}^{t} \mathrm{~L}\right)^{\circ}$,

(ii) Uma das seguintes situações ocorre:

(ii.1) $a^{-1}(0)=\mathbb{T}^{1}$

(ii.2) $a^{-1}(0)=\emptyset$ e $\mu=(2 \pi)^{-1} \int_{\mathbb{T}^{1}}(1 / a)$ é racional ou irracional não Liouville;

(ii.3) $\emptyset \neq a^{-1}(0) \neq \mathbb{T}^{1}$ e cada $x \in a^{-1}(0)$ é um zero de ordem finita. 
Novamente, quando $a^{-1}(0)=\mathbb{T}^{1}$, temos um campo vetorial com coeficientes constantes e o teorema anterior segue do Teorema (A.1) do Apêndice A.

Como na seção anterior, no caso em que $a^{-1}(0)=\emptyset$, um operador do tipo $L$ também pode ser reduzido a um operador com coeficientes constantes. Observe que verificar se $L$ agindo em $\mathcal{D}^{\prime}\left(\mathbb{T}^{2}\right)$ possui imagem fechada equivale a verificar se $L$ agindo em $\mathcal{P}_{2 \pi}^{\prime}\left(\mathbb{R}^{2}\right)$ possui imagem fechada. Considerando os transpostos ${ }^{t} \mathcal{M}$ e ${ }^{t} \mathcal{M}^{-1}$ dos homeomorfismos lineares $\mathcal{M}$ e $\mathcal{M}^{-1}$, definidos na seção anterior, temos ${ }^{t} \mathcal{M} \circ \mathrm{L} \circ{ }^{t} \mathcal{M}^{-1}=a \tilde{\mathrm{L}}$, onde $\tilde{\mathrm{L}}=\partial_{X}+\mu \partial_{T}$. Como $a^{-1}(0)=\emptyset$, o operador $\mathrm{L}$ possui imagem fechada se, e somente se, $\tilde{L}$ possui imagem fechada, o que ocorre se, e somente se, $\mu$ é racional ou irracional não Liouville, conforme Teorema (A.1) do Apêndice A. Mais ainda, das considerações feitas no Apêndice A segue que se $a^{-1}(0)=\mathbb{T}^{1}$ ou $a^{-1}(0)=\emptyset$, então L não será sobrejetor.

Utilizando o estudo dos operadores do tipo P efetuado na seção anterior, mostraremos que quando $\emptyset \neq a^{-1}(0) \neq \mathbb{T}^{1}$, a condição de $a^{-1}(0)$ conter apenas zeros de ordem finita será suficiente para que o operador L seja sobrejetor.

3.16 Teorema. Se $\emptyset \neq a^{-1}(0) \neq \mathbb{T}^{1}$ e $a^{-1}(0)$ contém apenas zeros de ordem finita, então $L \mathcal{D}^{\prime}\left(\mathbb{T}^{2}\right)=\mathcal{D}^{\prime}\left(\mathbb{T}^{2}\right)$.

Prova: Segue dos teoremas (3.7) e (3.12) que $P: \mathcal{C}^{\infty}\left(\mathbb{T}^{2}\right) \rightarrow \mathcal{C}^{\infty}\left(\mathbb{T}^{2}\right)$ possui imagem fechada e como $L=-{ }^{t} \mathrm{P}$, segue da Proposição (1.3) que $L \mathcal{D}^{\prime}\left(\mathbb{T}^{2}\right)$ é um subespaço fechado na topologia fraca*. Pela Proposição (1.2) segue que ${ }^{\circ}(\operatorname{ker} \mathrm{P})=\mathrm{LD}^{\prime}\left(\mathbb{T}^{2}\right)$.

Por fim, mostraremos que ker $\mathrm{P}=\{0\}$. De fato, tome $u \in$ ker $\mathrm{P}$. Utilizando série parcial de Fourier segue que $\mathrm{P} u=0$ se, e somente se, $\left(a \hat{u}_{k}\right)^{\prime}+i k \hat{u}_{k}=0$, para todo $k \in \mathbb{Z}$. Como na seção anterior, escolha $x_{1} \in a^{-1}(0)$, denote o conjunto $a^{-1}(0) \cap\left[x_{1}, x_{1}+2 \pi\right)$ por $\left\{x_{1}<\cdots<x_{N}\right\}$ e defina $x_{N+1}=x_{1}+2 \pi$. Fixe $j \in\{1, \ldots, N\}$ e $y_{j} \in\left(x_{j}, x_{j+1}\right)$. Utilizando fator integrante obtemos $\left(a \hat{u}_{k}\right)(x)=c \exp \left\{-i k \int_{y_{j}}^{x} 1 / a(y) d y\right\}, x \in\left(x_{j}, x_{j+1}\right)$. Em particular, $\left|a(x) \hat{u}_{k}(x)\right| \equiv|c|$ em $\left(x_{j}, x_{j+1}\right)$ e da continuidade das funções $a$ e $\hat{u}_{k}$ obtemos $c=0$. Assim, $\hat{u}_{k} \equiv 0$ para todo $k \in \mathbb{Z}$, ou seja, $u \equiv 0$. Portanto, ker $\mathrm{P}=\{0\}$ e $\operatorname{LD}^{\prime}\left(\mathbb{T}^{2}\right)=\mathcal{D}^{\prime}\left(\mathbb{T}^{2}\right)$

A próxima etapa será provar que quando $\emptyset \neq a^{-1}(0) \neq \mathbb{T}^{1}$, então a finitude da ordem dos zeros de a será uma condição necessária para que $L \mathcal{D}^{\prime}\left(\mathbb{T}^{2}\right)$ seja um subespaço fechado de $\mathcal{D}^{\prime}\left(\mathbb{T}^{2}\right)$. Para tanto, considere a restrição, $L_{0}$, de $L$ ao subespaço $\mathcal{D}^{\prime}\left(\mathbb{T}^{1}\right) \simeq \mathcal{D}^{\prime}\left(\mathbb{T}^{1}\right) \otimes 1_{t}$. Um cálculo direto mostra que

$$
\mathrm{L}_{0}: \mathcal{D}^{\prime}\left(\mathbb{T}^{1}\right) \rightarrow \mathcal{D}^{\prime}\left(\mathbb{T}^{1}\right)
$$


é dado por

$$
\mathrm{L}_{0}=a(d / d x)
$$

Para saber que a imagem de $L$ não é um subespaço fechado basta saber que a imagem de $\mathrm{L}_{0}$ não é um subespaço fechado, isto é o conteúdo da próxima proposição.

3.17 Proposição. Se $\mathrm{LD}^{\prime}\left(\mathbb{T}^{2}\right)$ for um subespaço fechado de $\mathcal{D}^{\prime}\left(\mathbb{T}^{2}\right)$, então $\mathrm{L}_{0} \mathcal{D}^{\prime}\left(\mathbb{T}^{1}\right)$ será um subespaço fechado de $\mathcal{D}^{\prime}\left(\mathbb{T}^{1}\right)$.

Prova: Suponha que $L_{0} \mathcal{D}^{\prime}\left(\mathbb{T}^{1}\right)$ não seja um subespaço fechado de $\mathcal{D}^{\prime}\left(\mathbb{T}^{1}\right)$. Segue da Proposição (1.2) que existe $u \in\left(\operatorname{ker}^{t} \mathrm{~L}_{0}\right)^{\circ} \backslash \mathrm{L}_{0} \mathcal{D}^{\prime}\left(\mathbb{T}^{1}\right)$. O operador

$$
\mathrm{P}_{0}: \mathcal{C}^{\infty}\left(\mathbb{T}^{1}\right) \rightarrow \mathcal{C}^{\infty}\left(\mathbb{T}^{1}\right)
$$

dado por

$$
\mathrm{P}_{0} \doteq d / d x(a \cdot)
$$

satisfaz ${ }^{t} \mathrm{P}_{0}=-\mathrm{L}_{0}$ e então ${ }^{t t} \mathrm{P}_{0}=-{ }^{t} \mathrm{~L}_{0}$. Como $\mathcal{C}^{\infty}\left(\mathbb{T}^{1}\right)$ é semireflexivo, segue da Proposição (1.5) que $u \in{ }^{\circ}\left(\operatorname{ker} \mathrm{P}_{0}\right) \backslash \mathrm{L}_{0} \mathcal{D}^{\prime}\left(\mathbb{T}^{1}\right)$. Observe que o operador $\mathrm{P}_{0}$ é a restrição do operador $\mathrm{P}=\partial_{t}+\partial_{x}(a \cdot): \mathcal{C}^{\infty}\left(\mathbb{T}^{2}\right) \rightarrow \mathcal{C}^{\infty}\left(\mathbb{T}^{2}\right)$ ao subespaço formado pelas funções que dependem apenas da variável $x$.

Consideremos $u \otimes 1_{t} \in \mathcal{D}^{\prime}\left(\mathbb{T}^{2}\right)$. Utilizando série parcial de Fourier em $\mathcal{C}^{\infty}\left(\mathbb{T}^{2}\right)$ obtemos

$$
\left\langle u \otimes 1_{t}, \phi\right\rangle=\left\langle u \otimes 1_{t}, \sum_{k \in \mathbb{Z}} \hat{\phi}_{k} e_{k}\right\rangle=\left\langle u \otimes 1_{t}, \hat{\phi}_{0} e_{0}\right\rangle=\left\langle 1_{t},\left\langle u, \hat{\phi}_{0}\right\rangle\right\rangle
$$

Assim, $\left\langle u \otimes 1_{t}, \phi\right\rangle=0$ para toda $\phi \in$ ker $\mathrm{P}$, uma vez que $\phi \in$ ker $\mathrm{P}$ implica que $\hat{\phi}_{0} \in$ ker $\mathrm{P}_{0}$. Portanto, $u \otimes 1_{t} \in{ }^{\circ}(\operatorname{ker} \mathrm{P})$ e novamente a Proposição (1.5) implica que ${ }^{\circ}(\operatorname{ker} \mathrm{P})=$ $\left(\operatorname{ker}^{t t} \mathrm{P}\right)^{\circ}=\left(\operatorname{ker}^{t} \mathrm{~L}\right)^{\circ}$, uma vez que $\mathcal{C}^{\infty}\left(\mathbb{T}^{2}\right)$ é semireflexivo.

Se mostrarmos que $u \otimes 1_{t} \notin \mathbf{L} \mathcal{D}^{\prime}\left(\mathbb{T}^{2}\right)$, seguirá da Proposição (1.2) que $\mathbf{L} \mathcal{D}^{\prime}\left(\mathbb{T}^{2}\right)$ não é um subespaço fechado de $\mathcal{D}^{\prime}\left(\mathbb{T}^{2}\right)$. Com efeito, se existir $v \in \mathcal{D}^{\prime}\left(\mathbb{T}^{2}\right)$ tal que $L v=u \otimes 1_{t}$, seguirá da série parcial de Fourier na variável $t$ que $a \hat{v}_{0}^{\prime}=u$ e $a \hat{v}_{k}^{\prime}+i k \hat{v}_{k}=0$ para todo $k \in \mathbb{Z} \backslash\{0\}$. Em particular, $\mathrm{L}_{0} \hat{v}_{0}=u$, uma contradição.

3.18 Observação. Sem usar que $\mathcal{C}^{\infty}\left(\mathbb{T}^{N}\right)$ é semireflexivo, o que se pode obter é que $\mathrm{L}_{0} \mathcal{D}^{\prime}\left(\mathbb{T}^{1}\right)$ será fechado na topologia fraca* de $\mathcal{D}^{\prime}\left(\mathbb{T}^{1}\right)$, sempre que $L \mathcal{D}^{\prime}\left(\mathbb{T}^{2}\right)$ for fechado na topologia fraca* $^{*}$ de $\mathcal{D}^{\prime}\left(\mathbb{T}^{2}\right)$. Para tanto utiliza-se a Proposição (1.4).

3.19 Teorema. Se $a^{-1}(0) \neq \mathbb{T}^{1}$ e existir um ponto em $\mathbb{T}^{1}$ onde a é flat, então a imagem de $\mathrm{L}_{0}$ não será fechada em $\mathcal{D}^{\prime}\left(\mathbb{T}^{1}\right)$. 
Prova: Vamos exibir uma distribuição em $\left(\operatorname{ker}^{t} \mathrm{~L}_{0}\right)^{\circ} \backslash \mathrm{L}_{0} \mathcal{D}^{\prime}\left(\mathbb{T}^{1}\right)$. Como $\mathcal{C}^{\infty}\left(\mathbb{T}^{1}\right)$ é semireflexivo, os mesmos argumentos da demonstração anterior implicam que basta exibir uma função em ${ }^{\circ}\left(\operatorname{ker} P_{0}\right) \backslash L_{0} \mathcal{D}^{\prime}\left(\mathbb{T}^{1}\right)$, onde o operador

$$
\mathrm{P}_{0}: \mathcal{C}^{\infty}\left(\mathbb{T}^{1}\right) \rightarrow \mathcal{C}^{\infty}\left(\mathbb{T}^{1}\right)
$$

é dado por

$$
\mathrm{P}_{0} \doteq d / d x(a \cdot)
$$

Para tanto, denote por $\mathcal{F} \subset \mathbb{T}^{1}$ o conjunto dos pontos onde $a$ é flat e seja $G \doteq \mathbb{T}^{1} \backslash \mathcal{F}$. Então $\mathcal{F}$ é fechado e, por hipótese, $\emptyset \neq \mathcal{F} \neq \mathbb{T}^{1}$, $\operatorname{logo} \emptyset \neq G \varsubsetneqq \mathbb{T}^{1}$ é um subconjunto aberto. Assim, as componentes conexas de $G$ são intervalos abertos da forma $(\alpha, \beta)$. Fixando uma componente conexa $(\alpha, \beta)$ de $G$ defina $f$ como a função característica de $(\alpha, \beta)$, isto é, $f=\chi_{(\alpha, \beta)}$ vale um em $(\alpha, \beta)$ e zero em $\mathbb{T}^{1} \backslash(\alpha, \beta)$.

Afirmamos que $f \in{ }^{\circ}\left(\operatorname{ker} \mathrm{P}_{0}\right)$. De fato, um cálculo utilizando a hipótese de que $a^{-1}(0) \neq \emptyset$ fornece que ker $\mathrm{P}_{0}=\left\{g \in \mathcal{C}^{\infty}\left(\mathbb{T}^{1}\right) ; a g=0\right\}$. Para cada $g \in$ ker $\mathrm{P}_{0}$, a identidade $a g=0$ e a continuidade de $g$ implicam que $g=0$ em $G$, uma vez que os zeros de $a$ em $G$, se existirem, são todos de ordem finita, logo isolados. Assim, para cada $g \in \operatorname{ker} \mathrm{P}_{0}$, temos $f g=0$ (como função) em $\mathbb{T}^{1}$ e, consequentemente, $\langle f, g\rangle=\langle g f, 1\rangle=0$. Portanto, $f \in{ }^{\circ}\left(\right.$ ker $\left.\mathrm{P}_{0}\right)$.

Por fim, o fato de $(\alpha, \beta)$ ser uma componente conexa de $G$, logo um conexo maximal, implica que a função $a$ é flat em $\{\alpha, \beta\}$. Segue da Proposição (2.45) que não pode existir $u \in \mathcal{D}^{\prime}\left(\mathbb{T}^{1}\right)$ tal que $a u=1 \mathrm{em}(\alpha, \beta)$. Assim, não pode existir $v \in \mathcal{D}^{\prime}\left(\mathbb{T}^{1}\right)$ tal que $\mathrm{L}_{0} v=a v^{\prime}=f=\chi_{(\alpha, \beta)}$, senão $u=v^{\prime}$ iria satisfazer $a u=1 \mathrm{em}(\alpha, \beta)$. Portanto, $f \notin \mathrm{L}_{0} \mathcal{D}^{\prime}\left(\mathbb{T}^{1}\right)$.

3.20 Observação. Sem utilizar que $\mathcal{C}^{\infty}\left(\mathbb{T}^{1}\right)$ é semireflexivo e utilizando a Proposição (1.4), os mesmos argumentos mostram que se $a^{-1}(0) \neq \mathbb{T}^{1}$ e se existir um ponto em $\mathbb{T}^{1}$ onde $a$ é flat, então a imagem de $\mathrm{L}_{0}$ não será fechada na topologia fraca* de $\mathcal{D}^{\prime}\left(\mathbb{T}^{1}\right)$ e da Observação (3.18) seguirá que a imagem de L não será fechada na topologia fraca* de $\mathcal{D}^{\prime}\left(\mathbb{T}^{1}\right)$.

Os teoremas (3.16), (3.17) e (3.19) finalizam a demonstração do Teorema (3.15) e junto com os resultados da seção anterior finalizam a demonstração do Teorema (3.1).

Segue das observações (3.18) e (3.20) que sem utilizar o fato de que $\mathcal{C}{ }^{\infty}\left(\mathbb{T}^{N}\right)$ é semireflexivo, podemos demonstrar que quando $\emptyset \neq a^{-1}(0) \neq \mathbb{T}^{1}$, a imagem de $\mathrm{L}$ será um subespaço fechado na topologia fraca* de $\mathcal{D}^{\prime}\left(\mathbb{T}^{2}\right)$ se, e somente se, a possuir apenas zeros de ordem finita. 
Para encerrar essa seção, finalizaremos a demonstração do Teorema (3.2). Suponha que $\mathrm{P}$ age em $\mathcal{C}^{\infty}\left(\mathbb{T}^{2}\right)$ e que $\emptyset \neq a^{-1}(0) \neq \mathbb{T}^{1}$. Se existir um ponto em $\mathbb{T}^{1}$ onde a função $a$ é flat, então $P \mathcal{C}^{\infty}\left(\mathbb{T}^{2}\right)$ não será um subespaço fechado de $\mathcal{C}^{\infty}\left(\mathbb{T}^{2}\right)$, uma vez que $P \mathcal{C}^{\infty}\left(\mathbb{T}^{2}\right)$ fechado em $\mathcal{C}^{\infty}\left(\mathbb{T}^{2}\right)$ implicaria que $-{ }^{t} \mathrm{P}=\mathrm{L}$ possuiria imagem fechada em $\mathcal{D}^{\prime}\left(\mathbb{T}^{2}\right)$ (veja a Proposição (1.3)), o que pelo Teorema (3.15) não pode ocorrer. Portanto, quando $\emptyset \neq a^{-1}(0) \neq \mathbb{T}^{1}$, a finitude da ordem dos zeros de a será uma condição necessária para que a imagem de $\mathrm{P}$ seja fechada em $\mathcal{C}^{\infty}\left(\mathbb{T}^{2}\right)$. Junto com os resultados da seção anterior, isso finaliza a demonstração do Teorema (3.4), isto é, do Teorema (3.2) quando consideramos o operador $\mathrm{P}$ agindo em $\mathcal{C}^{\infty}\left(\mathbb{T}^{2}\right)$.

Agora considere $\mathrm{P}$ agindo em $\mathcal{D}^{\prime}\left(\mathbb{T}^{2}\right)$. Analogamente ao que ocorre com os campos da forma $\mathrm{L}$, quando $a^{-1}(0)=\emptyset$ o estudo da resolubilidade global de um operador do tipo $\mathrm{P}$ se reduz ao estudo da resolubilidade global de um campo vetorial com coeficientes constantes da forma $\tilde{\mathrm{L}}=\partial_{X}+\mu \partial_{T}$, logo o Teorema (3.2) é uma consequência do Teorema (A.1) do Apêndice A. Mais ainda, quando $a^{-1}(0)=\mathbb{T}^{1}$ ou $a^{-1}(0)=\emptyset$, as considerações feitas no Apêndice A também permitem concluir que nenhum campo da forma $P$ será sobrejetor.

Resta provar que quando $\emptyset \neq a^{-1}(0) \neq \mathbb{T}^{1}$, a condição de $a^{-1}(0)$ conter apenas zeros de ordem finita será necessária e suficiente para a resolubilidade global de $\mathrm{P}$ em $\mathcal{D}^{\prime}\left(\mathbb{T}^{2}\right)$.

3.21 Teorema. Se $\emptyset \neq a^{-1}(0) \neq \mathbb{T}^{1}$ e $a^{-1}(0)$ contém apenas zeros de ordem finita, então $\mathrm{PD}^{\prime}\left(\mathbb{T}^{2}\right)=\mathcal{D}^{\prime}\left(\mathbb{T}^{2}\right)$.

Prova: Do Teorema (3.1) segue que o operador

$$
\mathrm{L}: \mathcal{C}^{\infty}\left(\mathbb{T}^{2}\right) \rightarrow \mathcal{C}^{\infty}\left(\mathbb{T}^{2}\right)
$$

tem imagem fechada em $\mathcal{C}^{\infty}\left(\mathbb{T}^{2}\right)$ e como

$$
\mathrm{P}=-{ }^{t} \mathrm{~L}: \mathcal{D}^{\prime}\left(\mathbb{T}^{2}\right) \rightarrow \mathcal{D}^{\prime}\left(\mathbb{T}^{2}\right)
$$

segue da Proposição (1.3) que $\mathrm{PD}^{\prime}\left(\mathbb{T}^{2}\right)$ é um subespaço fechado na topologia fraca* Pela Proposição (1.2) segue que ${ }^{\circ}(\operatorname{ker} \mathrm{L})=\mathrm{PD}^{\prime}\left(\mathbb{T}^{2}\right)$.

Por fim, mostraremos que ker $\mathrm{L}=\{0\}$. De fato, para cada $u \in \mathcal{C}^{\infty}\left(\mathbb{T}^{2}\right)$, utilizando série parcial de Fourier obtemos $u \in$ ker $L$ se, e somente se, $a \hat{u}_{k}^{\prime}+i k \hat{u}_{k}=0$, para todo $k \in \mathbb{Z}$.

Como na seção anterior, escolha $x_{1} \in a^{-1}(0)$, denote o conjunto $a^{-1}(0) \cap\left[x_{1}, x_{1}+2 \pi\right)$ por $\left\{x_{1}<\cdots<x_{N}\right\}$ e defina $x_{N+1}=x_{1}+2 \pi$. Fixe $j \in\{1, \ldots, N\}$ e $y_{j} \in\left(x_{j}, x_{j+1}\right)$. 
Utilizando fator integrante obtemos $\hat{u}_{k}(x)=c \exp \left\{-i k \int_{y_{j}}^{x} 1 / a(y) d y\right\}, x \in\left(x_{j}, x_{j+1}\right)$. Em particular, $\left|\hat{u}_{k}(x)\right| \equiv c$, para $x \in\left(x_{j}, x_{j+1}\right)$ e pela continuidade do coeficiente parcial $\hat{u}_{k}$ segue que $c=0$. Assim, $\hat{u}_{k} \equiv 0$ para todo $k \in \mathbb{Z}$, ou seja, $u \equiv 0$. Portanto, ker $\mathrm{L}=\{0\}$ e $\mathrm{PD}^{\prime}\left(\mathbb{T}^{2}\right)=\mathcal{D}^{\prime}\left(\mathbb{T}^{2}\right)$

Consideremos agora a restrição $\mathrm{P}_{0}$ de $\mathrm{P}$ ao subespaço $\mathcal{D}^{\prime}\left(\mathbb{T}^{1}\right) \simeq \mathcal{D}^{\prime}\left(\mathbb{T}^{1}\right) \otimes 1_{t}$. Um cálculo direto mostra que

$$
\mathrm{P}_{0}: \mathcal{D}^{\prime}\left(\mathbb{T}^{1}\right) \rightarrow \mathcal{D}^{\prime}\left(\mathbb{T}^{1}\right)
$$

é dado por

$$
\mathrm{P}_{0}=d / d x(a \cdot)
$$

Para saber que a imagem de P não é um subespaço fechado basta saber que a imagem de $\mathrm{P}_{0}$ não é um subespaço fechado, conforme enunciamos abaixo.

3.22 Teorema. Se $\mathrm{PD}^{\prime}\left(\mathbb{T}^{2}\right)$ for um subespaço fechado de $\mathcal{D}^{\prime}\left(\mathbb{T}^{2}\right)$, então $\mathrm{P}_{0} \mathcal{D}^{\prime}\left(\mathbb{T}^{1}\right)$ será um subespaço fechado de $\mathcal{D}^{\prime}\left(\mathbb{T}^{1}\right)$.

A demonstração do teorema acima segue a mesma técnica que foi utilizada na demonstração do Teorema (3.17) e será omitida.

3.23 Teorema. Se $\emptyset \neq a^{-1}(0) \neq \mathbb{T}^{1}$ e a for flat em algum ponto, então a imagem de $\mathrm{P}_{0}$ não será um subespaço fechado de $\mathcal{D}^{\prime}\left(\mathbb{T}^{1}\right)$.

Prova: Basta mostrar que existe uma distribuição no conjunto $\left(\text { ker }{ }^{t} \mathrm{P}_{0}\right)^{\circ} \backslash \mathrm{P}_{0} \mathcal{D}^{\prime}\left(\mathbb{T}^{1}\right)$. Considerando o operador $L$ agindo em $\mathcal{C}^{\infty}\left(\mathbb{T}^{2}\right)$ e restringindo $L$ ao subespaço de $\mathcal{C}^{\infty}\left(\mathbb{T}^{2}\right)$ formado pelas funções que dependem apenas da variável $x$, obtemos

$$
\mathrm{L}_{0}: \mathcal{C}^{\infty}\left(\mathbb{T}^{1}\right) \rightarrow \mathcal{C}^{\infty}\left(\mathbb{T}^{1}\right)
$$

e

$$
-{ }^{t} \mathrm{~L}_{0}=\mathrm{P}_{0}: \mathcal{D}^{\prime}\left(\mathbb{T}^{1}\right) \rightarrow \mathcal{D}^{\prime}\left(\mathbb{T}^{1}\right),
$$

$\operatorname{logo}-{ }^{t t} \mathrm{~L}_{0}={ }^{t} \mathrm{P}_{0}$. Como $\mathcal{C}^{\infty}\left(\mathbb{T}^{1}\right)$ é semireflexivo, segue da Proposição (1.5) que basta exibir uma distribuição no conjunto ${ }^{\circ}\left(\operatorname{ker} \mathrm{L}_{0}\right) \backslash \mathrm{P}_{0} \mathcal{D}^{\prime}\left(\mathbb{T}^{1}\right)$.

Denote por $\mathcal{F} \subset \mathbb{T}^{1}$ o conjunto dos pontos onde a é flat e seja $G \doteq \mathbb{T}^{1} \backslash \mathcal{F}$. Então $\mathcal{F}$ é fechado e, por hipótese, $\emptyset \neq \mathcal{F} \neq \mathbb{T}^{1} ; \log \emptyset \neq G \varsubsetneqq \mathbb{T}^{1}$ é um subconjunto aberto. Assim, as componentes conexas de $G$ são intervalos abertos da forma $(\alpha, \beta)$. Fixando uma componente conexa $(\alpha, \beta)$ de $G$, considere a distribuição $\delta(x-\alpha)$. 
Afirmamos que $\delta^{\prime}(x-\alpha) \in{ }^{\circ}\left(\operatorname{ker} \mathrm{L}_{0}\right) \subset \mathcal{D}^{\prime}\left(\mathbb{T}^{1}\right)$. De fato, se $g \in \operatorname{ker} \mathrm{L}_{0}$, então $a g^{\prime}=0$ e como os zeros de $a$ em $G$, se existirem, são isolados, segue da continuidade de $g^{\prime}$ que $g^{\prime}(\alpha)=0$. Portanto, $\left\langle\delta^{\prime}(x-\alpha), g\right\rangle=-\left\langle\delta(x-\alpha), g^{\prime}\right\rangle=-g^{\prime}(\alpha)=0$, para toda $g \in$ ker $\mathrm{L}_{0}$.

Por fim, o fato de $(\alpha, \beta)$ ser uma componente conexa de $G$, logo um conexo maximal, implica que a função a é flat em $\{\alpha, \beta\}$. Assim, não pode existir $u \in \mathcal{D}^{\prime}\left(\mathbb{T}^{1}\right)$ tal que $\mathrm{P}_{0} u=(a u)^{\prime}=\delta^{\prime}(x-\alpha)$, senão teríamos $a u=\delta(x-\alpha)+c$, o que pela Proposição $(2.46)$ não ocorre, uma vez que $a$ é flat em $\alpha$.

Segue dos teoremas (3.22) e (3.23) que quando $\emptyset \neq a^{-1}(0) \neq \mathbb{T}^{1}$, a finitude da ordem de anulamento dos zeros de $a$ será necessária para a resolubilidade global em $\mathcal{D}^{\prime}\left(\mathbb{T}^{2}\right)$ de um operador do tipo P, o que finaliza a demonstração do Teorema (3.2). 

Apêndice

\section{$A$}

\section{Campos vetoriais com coeficientes constantes no toro bidimensional}

Dedicamos este apêndice ao estudo da resolubilidade global de campos vetoriais com coeficientes constantes em $\mathbb{T}_{(x, t)}^{2}$, a saber, $\tilde{L}=\partial_{x}+\alpha \partial_{t}$, onde $\alpha \in \mathbb{C}$. As referências para a resolubilidade global de campos vetoriais com coeficientes constantes em $\mathbb{T}^{2}$ são [10] e [8]. A resolubilidade global dos campos vetoriais do tipo L está intimamente relacionada com a natureza do número complexo $\alpha \in \mathbb{C}$. Analisaremos também quais operadores da forma $\tilde{L}$ são sobrejetores e quais são fortemente resolúveis.

A.1 Teorema. Consideremos $\tilde{L}=\partial_{x}+\alpha \partial_{t}$, onde $\alpha \in \mathbb{C}$, um campo vetorial com coeficientes constantes. Então as seguintes propriedades são equivalentes:

(I) $\tilde{\mathrm{L}} \mathcal{C}^{\infty}\left(\mathbb{T}^{2}\right)$ é um subespaço fechado em $\mathcal{C}^{\infty}\left(\mathbb{T}^{2}\right)$,

(II) $\tilde{\mathrm{L}} \mathcal{D}^{\prime}\left(\mathbb{T}^{2}\right)$ é um subespaço fechado em $\mathcal{D}^{\prime}\left(\mathbb{T}^{2}\right)$,

(III) Uma das seguintes situações ocorre:

(III.1) $\alpha \in \mathbb{C} \backslash \mathbb{R}$

(III.2) $\alpha \in \mathbb{R} \backslash \mathbb{Q}$ e $\alpha$ não é um número de Liouville;

(III.3) $\alpha \in \mathbb{Q}$. 
Prova: Consideremos inicialmente $\tilde{L}$ agindo em $\mathcal{C}^{\infty}\left(\mathbb{T}^{2}\right)$.

(Caso III.1). Suponha que $\alpha \in \mathbb{C} \backslash \mathbb{R}$.

Se $\alpha=i b$, onde $b$ é um número real não nulo, dada $f \in\left(\operatorname{ker}^{t} \tilde{\mathbf{L}}\right)^{\circ}$ defina

$$
u(x, t)=\sum_{(j, k) \in \mathbb{Z}^{2} \backslash\{0\}} \frac{\hat{f}(j, k)}{i(j+\alpha k)} e^{(i j x+i k t)},
$$

para todo $(x, t) \in \mathbb{T}^{2}$. Como $\left|(j+\alpha k)^{-1} \hat{f}(j, k)\right| \leq 2|\hat{f}(j, k)|(\min \{1,|b|\})^{-1}(|j|+|k|)^{-1} \leq$ $2|\hat{f}(j, k)|(\min \{1,|b|\})^{-1}$, para todo $(j, k) \in \mathbb{Z}^{2} \backslash\{0\}$ e do fato de que $\hat{f}(j, k)$ decresce rápido segue que a sequência $[i(j+\alpha k)]^{-1} \hat{f}(j, k)$ é rapidamente decrescente, logo a função $u \in \mathcal{C}^{\infty}\left(\mathbb{T}^{2}\right)$ satisfaz $i(j+\alpha k) \hat{u}(j, k)=\hat{f}(j, k)$ para todo $(j, k) \in \mathbb{Z}^{2} \backslash\{0\}$. Note que a distribuição $1_{x} \otimes 1_{t}$ pertence a $\operatorname{ker}^{t} \tilde{\mathbf{L}}$, pois ${ }^{t} \tilde{\mathbf{L}}=-\tilde{\mathrm{L}}$. Assim, $(2 \pi)^{2} \hat{f}(0,0)=\left\langle 1_{x} \otimes 1_{t}, f\right\rangle=0$ e, portanto, $\tilde{\mathrm{L}} u=f$.

Seja $\alpha=b_{1}+i b_{2}$, onde $b_{1}$ e $b_{2}$ são números reais não nulos. Então $|j+\alpha k| \geq\left|j+b_{1} k\right|$ e $|j+\alpha k| \geq\left|b_{2} k\right|$. Tomando $0<\epsilon<1$ temos $\left|j+b_{1} k\right| \leq \epsilon|j|$ ou $\left|j+b_{1} k\right| \geq \epsilon|j|$. Se $\left|j+b_{1} k\right| \geq \epsilon|j|$, então $2|j+\alpha k| \geq \epsilon|j|+\left|b_{2}\right||k| \geq \min \left\{\epsilon,\left|b_{2}\right|\right\}(|j|+|k|)$. Senão, $\left|j+b_{1} k\right| \leq \epsilon|j|$ e consequentemente $\left|b_{1} k\right| \geq|j|(1-\epsilon), \operatorname{logo}|j+\alpha k| \geq\left|b_{2} k\right|=\frac{1}{2}\left(\left|b_{2}\right||k|+\right.$ $\left.\frac{\left|b_{2}\right|}{\left|b_{1}\right|}\left|b_{1}\right||k|\right) \geq \frac{\left|b_{2}\right||k|}{2}+\frac{\left|b_{2}\right|}{2\left|b_{1}\right|}(1-\epsilon)|j| \geq \min \left\{\frac{1}{2}, \frac{\left|b_{2}\right|}{2\left|b_{1}\right|}(1-\epsilon)\right\}(|j|+|k|)$. Em ambos os casos existe um número real $C>0$ satisfazendo $|j+\alpha k| \geq C(|j|+|k|)$, para todo $(j, k) \in \mathbb{Z}^{2}$. Portanto, dada uma função $f \in\left(\operatorname{ker}^{t} \tilde{\mathbf{L}}\right)^{\circ}$, defina

$$
u(x, t)=\sum_{(j, k) \in \mathbb{Z}^{2} \backslash\{0\}} \frac{\hat{f}(j, k)}{i(k+\alpha k)} e^{(i j x+i k t)}
$$

para todo $(x, t) \in \mathbb{T}^{2}$. Como $\left|(j+\alpha k)^{-1} \hat{f}(j, k)\right| \leq C^{-1}|\hat{f}(j, k)|(|j|+|k|)^{-1} \leq C^{-1}|\hat{f}(j, k)|$, para todo $(j, k) \in \mathbb{Z}^{2} \backslash\{0\}$, segue que a sequência $[i(j+\alpha k)]^{-1} \hat{f}(j, k)$ é rapidamente decrescente, logo $u \in \mathcal{C}^{\infty}\left(\mathbb{T}^{2}\right)$ satisfaz $i(j+\alpha k) \hat{u}(j, k)=\hat{f}(j, k)$ para todo $(j, k) \in \mathbb{Z}^{2} \backslash\{0\}$ e como $\hat{f}(0,0)=0$ temos $\tilde{\mathrm{L}} u=f$.

(Caso III.2). Seja $\alpha \in \mathbb{R} \backslash \mathbb{Q}$. Da definição de número de Liouville segue que $\alpha$ não é um número de Liouville se, e somente se, existem constantes $c_{1}, c_{2}, c_{3}>0$ tais que $|j+\alpha k| \geq c_{1}(|j|+|k|)^{-c_{2}}$, para todo $(j, k) \in \mathbb{Z}^{2}$ com $|j|+|k| \geq c_{3}$. Esta condição aparece na referência [8].

Suponha que $\alpha$ não seja um número de Liouville, isto é, que existam constantes $c_{1}, c_{2}, c_{3}>0$ tais que $|j+\alpha k| \geq c_{1}(|j|+|k|)^{-c_{2}}$, para todo $(j, k) \in \mathbb{Z}^{2}$ com $|j|+|k| \geq c_{3}$. 
Dada $f \in\left(\operatorname{ker}^{t} \tilde{\mathrm{L}}\right)^{\circ}$, defina $\left(c_{j k}\right)_{(j, k) \in \mathbb{Z}^{2}}$ por $c_{j k}=\hat{f}(j, k)[i(j+\alpha k)]^{-1}$, para todo $(j, k) \neq$ $(0,0)$ e $c_{00}=0$.

Afirmamos que a sequência $\left(c_{j k}\right)_{(j, k) \in \mathbb{Z}^{2}}$ é rapidamente decrescente. De fato, dado $r>0$, existe $c>0$ tal que $|\hat{f}(j, k)| \leq c(|j|+|k|)^{-r-c_{2}}$, para todo $(j, k) \in \mathbb{Z}^{2} \backslash\{(0,0)\}$, $\operatorname{logo}\left|c_{j k}\right|=|\hat{f}(j, k)||j+\alpha k|^{-1} \leq c c_{1}^{-1}(|j|+|k|)^{-r}$, para todo $(j, k) \in \mathbb{Z}^{2} \backslash\{(0,0)\}$. Portanto, a sequência $\left(c_{j k}\right)_{(j, k) \in \mathbb{Z}^{2}}$ é rapidamente decrescente, logo define uma função $u \in \mathcal{C}^{\infty}\left(\mathbb{T}^{2}\right)$ tal que $\hat{u}(j, k)=c_{j k}$ para todo $(j, k) \in \mathbb{Z}^{2}$. Assim, $u \in \mathcal{C}^{\infty}\left(\mathbb{T}^{2}\right)$ satisfaz $i(j+\alpha k) \hat{u}(j, k)=\hat{f}(j, k)$ para todo $(j, k) \in \mathbb{Z}^{2} \backslash\{0\}$ e como $f \in\left(\operatorname{ker}^{t} \tilde{\mathbf{L}}\right)^{\circ}$ implica que $\hat{f}(0,0)=0$, concluímos que $u \in \mathcal{C}^{\infty}\left(\mathbb{T}^{2}\right)$ satisfaz $\tilde{\mathrm{L}} u=f$.

Reciprocamente, suponha que $\alpha$ seja um número de Liouville. Mostraremos que existe $f \in\left(\operatorname{ker}^{t} \tilde{\mathrm{L}}\right)^{\circ} \backslash \mathcal{C}^{\infty}\left(\mathbb{T}^{2}\right)$. Cálculos diretos mostram que $\left\{\phi \in \mathcal{C}^{\infty}\left(\mathbb{T}^{2}\right) ; \hat{\phi}(0)=0\right\}=$ $\left(\operatorname{ker}^{t} \tilde{\mathrm{L}}\right)^{\circ}$. Para cada $n \in \mathbb{N}$ existe $\left(j_{n}, k_{n}\right) \in \mathbb{Z}^{2}$ tal que $\left|j_{n}\right|+\left|k_{n}\right| \geq n$ e $\left|j_{n}+\alpha k_{n}\right|<$ $\left(\left|j_{n}\right|+\left|k_{n}\right|\right)^{-n}$. Como a sequência $\left(\left(\left|j_{n}\right|+\left|k_{n}\right|\right)^{\frac{-n}{2}}\right)_{n \in \mathbb{N}}$ é rapidamente decrescente, fica bem definida uma função $f \in\left(\operatorname{ker}^{t} \tilde{\mathrm{L}}\right)^{\circ}$ dada por

$$
f(x, t)=\sum_{n=1}^{\infty}\left(\left|j_{n}\right|+\left|k_{n}\right|\right)^{\frac{-n}{2}} \exp \left(i j_{n} x+i k_{n} t\right)
$$

para todo $(x, t) \in \mathbb{T}^{2}$.

Agora suponha que exista $u \in \mathbb{T}^{2}$ satisfazendo $\tilde{\mathrm{L}} u=f$. Utilizando coeficientes de Fourier concluímos que $i(j+\alpha k) \hat{u}(j, k)=\hat{f}(j, k)$, para todo $(j, k) \in \mathbb{Z}^{2}$. Em particular, para cada $n \in \mathbb{N}$, temos $\left|\hat{u}\left(j_{n}, k_{n}\right)\right|=\left|\hat{f}\left(j_{n}, k_{n}\right)\right|\left|i\left(j_{n}+\alpha k_{n}\right)\right|^{-1}>\left(\left|j_{n}\right|+\left|k_{n}\right|\right)^{\frac{n}{2}}$. Verificase que a sequência $\left(\left(\left|j_{n}\right|+\left|k_{n}\right|\right)^{\frac{n}{2}}\right)_{n \in \mathbb{N}}$ não é rapidamente decrescente. Portanto, temos uma contradição.

(Caso III.3). Suponha agora que $\alpha \in \mathbb{Q}$. Se $\alpha \neq 0$ podemos escrever $\alpha=p / q$, fração irredutível, com $p \in \mathbb{Z}$ e $q \in \mathbb{N}$. Se $\alpha=0$ escrevemos $\alpha=0 / 1$. Defina o conjunto $\mathcal{A}=\left\{(j, k) \in \mathbb{Z}^{2} ; j+\alpha k=0\right\}=\left\{(j, k) \in \mathbb{Z}^{2} ; j=p \ell, k=-q \ell, \ell \in \mathbb{Z}\right\}$ e seja $\mathcal{B}=\mathbb{Z}^{2} \backslash \mathcal{A}$.

Utilizando série de Fourier verifica-se que $\left\{v \in \mathcal{D}^{\prime}\left(\mathbb{T}^{2}\right) ; \hat{v}(j, k)=0\right.$ se $\left.(j, k) \in \mathcal{B}\right\}=$ $\operatorname{ker}^{t} \tilde{\mathrm{L}}$ e que $\left(\operatorname{ker}^{t} \tilde{\mathrm{L}}\right)^{\circ}=\left\{\phi \in \mathcal{C}^{\infty}\left(\mathbb{T}^{2}\right) ; \hat{\phi}(j, k)=0\right.$, se $\left.(j, k) \in \mathcal{A}\right\}$. Sendo assim, dada $f \in$ $\left(\operatorname{ker}^{t} \tilde{\mathrm{L}}\right)^{\circ}$, definimos a sequência $\left(c_{j k}\right)_{(j, k) \in \mathbb{Z}^{2}}$ por $c_{j k}=\hat{f}(j, k)[i(j+\alpha k)]^{-1}$, se $(j, k) \in \mathcal{B}$ e $c_{j k}=0$, se $(j, k) \in \mathcal{A}$. Por definição, se $(j, k) \in \mathcal{B}$ então $j+\alpha k \neq 0$, logo $q j+p k \neq 0$. Como $q j+p k \in \mathbb{Z}$, resulta que $|q j+p k| \geq 1$ e, portanto, $|j+\alpha k| \geq 1 / q$. Daqui seguese facilmente que a sequência $\left(c_{j k}\right)_{(j, k) \in \mathbb{Z}^{2}}$ é rapidamente decrescente, logo define uma função $u \in \mathcal{C}^{\infty}\left(\mathbb{T}^{2}\right)$ que satisfaz $i(j+\alpha k) \hat{u}(j, k)=i(j+\alpha k) c_{j k}=\hat{f}(j, k)$, para todo $(j, k) \in \mathbb{Z}^{2}$, ou seja, $\tilde{\mathrm{L}} u=f$. 
90 Apêndice A - Campos vetoriais com coeficientes constantes no toro bidimensional

As considerações anteriores finalizam a demonstração do Teorema (A.1) quando consideramos o campo $\tilde{L}$ agindo em $\mathcal{C}^{\infty}\left(\mathbb{T}^{2}\right)$. Para a demonstração do caso em que $\tilde{L}$ age em $\mathcal{D}^{\prime}\left(\mathbb{T}^{2}\right)$ utiliza-se tais técnicas mais a Proposição (1.5) (aqui usa que $\mathcal{C}^{\infty}\left(\mathbb{T}^{2}\right)$ é semireflexivo).

Vale observar que se $\tilde{L}$ age em $\mathcal{C}^{\infty}\left(\mathbb{T}^{2}\right)$, então $\operatorname{ker}^{t} \tilde{L} \neq\{0\}$ implica que $\tilde{L}$ não é sobrejetor. Utilizando série de Fourier verifica-se que:

1. Se $\alpha \in \mathbb{C} \backslash \mathbb{Q}$, então $\operatorname{ker}^{t} \tilde{\mathbf{L}}=\left\{v \in \mathcal{D}^{\prime}\left(\mathbb{T}^{2}\right) ; \hat{v}(j, k)=0\right.$, se $\left.(j, k) \in \mathbb{Z}^{2} \backslash\{0\}\right\}$.

2. Se $\alpha \in \mathbb{Q}$, então $\operatorname{ker}^{t} \tilde{\mathrm{L}}=\left\{v \in \mathcal{D}^{\prime}\left(\mathbb{T}^{2}\right) ; \hat{v}(j, k)=0\right.$, se $\left.(j, k) \in \mathcal{B}\right\}$, onde $\mathcal{B}$ é definido na demonstração do Teorema (A.1) (caso III.3).

Portanto, nenhum campo da forma $\tilde{L}$ agindo em $\mathcal{C}^{\infty}\left(\mathbb{T}^{2}\right)$ é sobrejetor.

Dos comentários após a Definição (1.2) do artigo [4] segue que um campo da forma $\tilde{\mathrm{L}}$ agindo em $\mathcal{C}^{\infty}\left(\mathbb{T}^{2}\right)$ será fortemente resolúvel se, e somente se, a imagem de $\tilde{\mathrm{L}}$ for um subespaço fechado e $\operatorname{ker}^{t} \tilde{L}$ for um subespaço de dimensão finita. Assim, do Teorema (A.1) e das propriedades acima resulta que $\tilde{L}$ é fortemente resolúvel se, e somente se, $\alpha \in \mathbb{C} \backslash \mathbb{R}$ ou $\alpha \in \mathbb{R} \backslash \mathbb{Q}$ e $\alpha$ não é um número de Liouville.

Por fim, se $\tilde{L}$ age em $\mathcal{D}^{\prime}\left(\mathbb{T}^{2}\right)$, então $\left(\operatorname{ker}^{t} \tilde{\mathrm{L}}\right) \neq\{0\}$ impica que $\tilde{\mathrm{L}}$ não é sobrejetor. Agora, $\left(\operatorname{ker}^{t} \tilde{\mathrm{L}}\right) \neq\{0\}$ se, e somente, se $\left(\operatorname{ker}^{t} \tilde{\mathrm{L}}\right)^{\circ} \neq \mathcal{D}^{\prime}\left(\mathbb{T}^{2}\right)$. Como $\mathcal{C}^{\infty}\left(\mathbb{T}^{2}\right)$ é semireflexivo, exigir que $\left(\operatorname{ker}^{t} \tilde{\mathrm{L}}\right)^{\circ} \neq \mathcal{D}^{\prime}\left(\mathbb{T}^{2}\right)$ é equivalente a exigir que ${ }^{\circ}(\operatorname{ker} \tilde{\mathrm{P}}) \neq \mathcal{D}^{\prime}\left(\mathbb{T}^{2}\right)$, onde

$$
\tilde{\mathrm{P}}=-\tilde{\mathrm{L}}: \mathcal{C}^{\infty}\left(\mathbb{T}^{2}\right) \rightarrow \mathcal{C}^{\infty}\left(\mathbb{T}^{2}\right)
$$

Como $\mathcal{C}^{\infty}\left(\mathbb{T}^{2}\right)$ é localmente convexo, uma aplicação do Teorema de Hahn-Banach fornece que $^{\circ}(\operatorname{ker} \tilde{\mathrm{P}}) \neq \mathcal{D}^{\prime}\left(\mathbb{T}^{2}\right)$ se, e somente se, ker $\tilde{\mathrm{P}} \neq\{0\}$.

Novamente, utilizando série de Fourier verifica-se que:

1. Se $\alpha \in \mathbb{C} \backslash \mathbb{Q}$, então ker $\tilde{\mathrm{P}}=\left\{\phi \in \mathcal{C}^{\infty}\left(\mathbb{T}^{2}\right) ; \hat{\phi}(j, k)=0\right.$, se $\left.(j, k) \in \mathbb{Z}^{2} \backslash\{0\}\right\}$.

2. Se $\alpha \in \mathbb{Q}$, então ker $\tilde{\mathrm{P}}=\left\{\phi \in \mathcal{C}^{\infty}\left(\mathbb{T}^{2}\right) ; \hat{\phi}(j, k)=0\right.$, se $\left.(j, k) \in \mathcal{B}\right\}$, onde $\mathcal{B}$ é definido na demonstração do Teorema (A.1) (caso III.3).

Portanto, para qualquer $\alpha \in \mathbb{C}$ segue que $\tilde{\mathrm{L}}$ agindo em $\mathcal{D}^{\prime}\left(\mathbb{T}^{2}\right)$ não é sobrejetor. 


\section{Referências Bibliográficas}

[1] Barros-Neto. - An introduction to the theory of distributions Marcel Dekker, INC. New York, 1973.

[2] Bergamasco, A. P. e Petronilho, G. - Closedness of the Range for Vector Fields on the Torus. Journal of Differential Equations. 154 (1999), 132-139.

[3] Bergamasco, A. P. e Dattori da Silva, P. L. - Global solvability for a special class of Vector Fields on the Torus. Contemporary Mathematics. 400 (2006), 11-20.

[4] Bergamasco, A. P. e Cordado, P. D. e Petronilho, G - Global Solvability for a Class of Complex Vector Fields on the Two-Torus. Communications in Partial Differential Equations. 29 (2004), 785-819.

[5] Elon, L. L. - Espaços Métricos, quarta edição. Projeto Euclides, IMPA, Rio de Janeiro, 2007.

[6] Elon, L. L. - Variedades Diferenciáveis, Publicações Matemáticas, IMPA, Rio de Janeiro, 2007.

[7] Friedlander, F. G. e Joshi, M. -Introduction to the theory of distributions. Cambridge University Press, 1982.

[8] Greenfield, S. e Wallach, N. - Global hypoellipticity and Liouville numbers. Proc. Amer. Math. Soc. 31 (1972), 112-114.

[9] Grubb, G. - Distributions and Operators. Springer, 2009.

[10] Herz, C. -Functions which are divergences. Amer. J. Math. 92 (1970), 641-656. 
[11] Hörmander, L. - The Analysis of Linear Partial Differential Operators I. SpringerVerlag, 1983.

[12] Hounie, J. - Teoria Elementar das Distribuições. $12^{\circ}$ Colóquio Brasileiro de Matemática, Poços de Caldas, 1979.

[13] Nirenberg, L. e Treves, F. - Solvability of a first order linear partial differential equation Comm. Pure Appl. Math. 16 (1963), 331-351.

[14] Reed, M. e Simon, B. - Methods of modern mathematical physics. vol I: Functional Analysis. Academic Press, New York, 1972.

[15] Rudin, W. - Functional Analysis. McGraw-Hill, 1973.

[16] Schaefer, H. H. - Topological Vector Spaces. Springer-Verlag, Third Printing Corrected, 1971.

[17] Treves, F. - Topological Vector Spaces, Distributions and Kernels. Academic Press, New York, 1967.

[18] Zani, S. L. - Hipoeliticidade global para operadores de segunda ordem. Dissertação de Mestrado, ICMC-USP, São Carlos, 1988. 
anulador, 14

coeficientes de Fourier, 55

coeficientes parciais de Fourier, 57

delta de Dirac, 28, 29

distribuição

composição com difeomorfismos, 30

de ordem finita, 29

definição de, 27

ordem da, 28, 29

periódica, 30

suporte, 33

espaço

de Fréchet, 16

reflexivo, 15

semireflexivo, 15

vetorial topológico, 13

exaustão de um aberto, 21

fórmula de Taylor, 20

número de Liouville, 60

operador

fortemente resolúvel, 59

globalmente resolúvel, 59

produto direto entre distribuições, 56

produto entre distribuições e funções, 31

regra de Leibniz, 20

série de Fourier, 55

série parcial de Fourier, 57

sequência

de crescimento lento, 54,57

rapidamente decrescente, 54,56

transposto, 15 



\begin{tabular}{|c|c|}
\hline$X^{\prime}, \quad 14$ & $\mathcal{C}^{\infty}(\Omega)^{\prime}, 27$ \\
\hline$X_{\sigma}^{\prime}, \quad 14$ & $\mathcal{P}_{T}^{\prime}\left(\mathbb{R}^{N}\right), 27$ \\
\hline$X_{b}^{\prime}, \quad 14$ & $\mathcal{D}^{\prime}(\Omega), 27$ \\
\hline${ }^{\circ} M, \quad 14$ & $\mathcal{C}_{c}^{\infty}(K), 28$ \\
\hline$N^{\circ}, \quad 14$ & $\mathcal{D}_{T}^{\prime}\left(\mathbb{R}^{N}\right), 30$ \\
\hline$J(x), 14$ & $H,{ }^{t} H, 30$ \\
\hline $\bar{Z}^{\omega^{*}}, \quad 15$ & $\tilde{H},{ }^{t} \tilde{H}, 30$ \\
\hline${ }^{t} T, \quad 15$ & $S(u), 33$ \\
\hline $\mathcal{R}(T), 16$ & $\mathcal{E}^{\prime}(\Omega), 33$ \\
\hline $\mathcal{C}^{\infty}(\Omega), 19$ & $\mathfrak{p}, \quad 47$ \\
\hline$\partial^{\alpha} \phi, \quad 19$ & $\mathbb{T}^{N}, \quad 47$ \\
\hline$S(f), 19$ & $\mathcal{C}^{\infty}\left(\mathbb{T}^{N}\right) 47$ \\
\hline $\mathcal{C}_{c}^{\infty}(\Omega), 19$ & $\Lambda, \quad 47$ \\
\hline $\mathcal{P}_{T}\left(\mathbb{R}^{N}\right), 19$ & $L^{p}\left(\mathbb{T}^{N}\right), 47$ \\
\hline$\alpha \leq \beta, 20$ & $\mathcal{D}^{\prime}\left(\mathbb{T}^{N}\right), 48$ \\
\hline$|\alpha|, \quad 20$ & $\otimes$ \\
\hline$x^{\alpha}$ & $\mu$ \\
\hline$\alpha !, \quad 20$ & $\mathcal{E}$ \\
\hline$(\beta), 20$ & $\mathcal{G}$ \\
\hline & $\mathcal{M}, \quad 62$ \\
\hline
\end{tabular}

